\title{
The bandwidth theorem for locally dense graphs
}

\author{
Katherine Staden ${ }^{1}$ and Andrew Treglown ${ }^{2}$ \\ ${ }^{1}$ Mathematical Institute, University of Oxford, Oxford, OX2 6GG United Kingdom; E-mail: staden@ maths.ox.ac.uk. \\ ${ }^{2}$ School of Mathematics, University of Birmingham, Edgbaston, Birmingham, B15 2TT United Kingdom; \\ E-mail: a.c.treglown@bham.ac.uk.
}

Received: 6 June 2019; Revised: 18 June 2020; Accepted: 16 June 2020

2020 Mathematics Subject Classification: Primary - 05C35

Keywords and phrases: bandwidth; embedding; regularity method

\begin{abstract}
The bandwidth theorem of Böttcher, Schacht, and Taraz [Proof of the bandwidth conjecture of Bollobás and Komlós, Mathematische Annalen, 2009] gives a condition on the minimum degree of an $n$-vertex graph $G$ that ensures $G$ contains every $r$-chromatic graph $H$ on $n$ vertices of bounded degree and of bandwidth $o(n)$, thereby proving a conjecture of Bollobás and Komlós [The Blow-up Lemma, Combinatorics, Probability, and Computing, 1999]. In this paper, we prove a version of the bandwidth theorem for locally dense graphs. Indeed, we prove that every locally dense $n$-vertex graph $G$ with $\delta(G)>(1 / 2+o(1)) n$ contains as a subgraph any given (spanning) $H$ with bounded maximum degree and sublinear bandwidth.
\end{abstract}

\section{Contents}

$1 \quad$ Introduction and results $\quad \mathbf{2}$

2 Overview of the proof of Theorem $1.2 \quad 3$

3 Preliminaries $\quad 5$

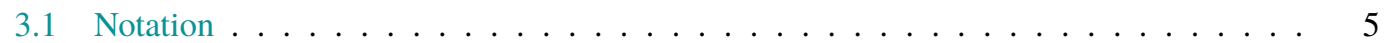

3.1 .1 Named graphs . . . . . . . . . . . . . . . . . . . . . 6

3.2 Properties of locally dense graphs . . . . . . . . . . . . . . 7

4 The regularity and blow-up lemmas and associated tools 9



4.2 Embedding lemmas . . . . . . . . . . . . . . . . 10

5 Findling the power of a Hamilton cycle $\quad 11$

6 Lemmas for $H \quad 15$

6.1 Partitioning a graph of low bandwidth: the basic lemma for $H \ldots \ldots \ldots$

6.2 Covering exceptional vertices: the second lemma for $H \ldots \ldots \ldots$. . . . . . . 18

7 The lemma for $G$ : adjusting cluster sizes $\quad 22$

8 The proof of Theorem $1.2 \quad 28$

9 Concluding remarks $\quad 34$

(c) The Author(s), 2020. Published by Cambridge University Press. This is an Open Access article, distributed under the terms of the Creative Commons Attribution licence (http://creativecommons.org/licenses/by/4.0/), which permits unrestricted re-use, distribution, and reproduction in any medium, provided the original work is properly cited. 


\section{Introduction and results}

One of the fundamental topics in extremal graph theory is the study of minimum degree conditions that force a graph to contain a given spanning substructure. Perhaps the best known result in the area is Dirac's theorem [13], which states that any graph $G$ on $n \geq 3$ vertices with minimum degree $\delta(G) \geq n / 2$ contains a Hamilton cycle. The Pósa-Seymour conjecture (see [15] and [33]) states that any graph $G$ on $n$ vertices with $\delta(G) \geq r n /(r+1)$ contains the $r$ th power of a Hamilton cycle. (The $r$ th power of a Hamilton cycle $C$ is obtained from $C$ by adding an edge between every pair of vertices of distance at most $r$ on $C$.) Komlós, Sárközy, and Szemerédi [28] proved this conjecture for sufficiently large graphs.

A decade ago, Böttcher, Schacht, and Taraz [9] proved a very general minimum degree result, the so-called bandwidth theorem. A graph $H$ on $n$ vertices is said to have bandwidth at most $b$ if there exists a labelling of the vertices of $H$ by the numbers $1, \ldots, n$ such that for every edge $i j \in E(H)$, we have $|i-j| \leq b$. Clearly, every graph $H$ has bandwidth at most $|H|-1$. Further, a Hamilton cycle has bandwidth 2 , and in general the $r$ th power of a Hamilton cycle has bandwidth at most $2 r$. Böttcher, Preussmann, Taraz, and Würfl [7] proved that every planar graph $H$ on $n$ vertices with bounded maximum degree has bandwidth at most $O(n / \log n)$. The bandwidth theorem gives a condition on the minimum degree of a graph $G$ on $n$ vertices that ensures $G$ contains every $r$-chromatic graph on $n$ vertices of bounded degree and of bandwidth $o(n)$.

Theorem 1.1 (The bandwidth theorem, Böttcher, Schacht, and Taraz [9]). Given any $r, \Delta \in \mathbb{N}$ and any $\gamma>0$, there exist constants $\beta>0$ and $n_{0} \in \mathbb{N}$ such that the following holds. Suppose that $H$ is an $r$-chromatic graph on $n \geq n_{0}$ vertices with $\Delta(H) \leq \Delta$ and bandwidth at most $\beta n$. If $G$ is a graph on $n$ vertices with

$$
\delta(G) \geq\left(\frac{r-1}{r}+\gamma\right) n,
$$

\section{then $G$ contains a copy of $H$.}

We remark that Theorem 1.1 had been conjectured by Bollobás and Komlós [26]. Since the bandwidth theorem was proven, a number of variants of the result have been obtained, including for arrangeable graphs [10] and degenerate graphs [30] and in the setting of random and pseudorandom graphs [1, 5, 23], as well as for robustly expanding graphs [24]. Very recently, a bandwidth theorem for approximate decompositions was proven by Condon, Kim, Kühn, and Osthus [12], whilst Glock and Joos [20] proved a $\mu n$-bounded edge colouring extension of Theorem 1.1. A general embedding result of Böttcher, Montgomery, Parczyk, and Person [6] also implies a bandwidth theorem in the setting of randomly perturbed graphs.

For many graphs $H$, the minimum degree condition in Theorem 1.1 is best-possible up to the term $\gamma n$. For example, suppose that $H$ is a $K_{r}$-factor (that is, we seek a collection of vertex-disjoint copies of $K_{r}$ in $G$ that together cover all the vertices in $G$ ). So $\chi(H)=r, \Delta(H)=r-1$, and $H$ has bandwidth $r-1$. Suppose that $G$ is obtained from two disjoint vertex classes $A$ and $B$ of sizes $n / r+1$ and $(r-1) n / r-1$, respectively, so that $G$ contains all edges other than those with both endpoints in $A$. Then it is easy to see that $G$ does not contain a $K_{r}$-factor; however, $\delta(G)=((r-1) / r) n-1$. In fact, note that the famous HajnalSzemerédi theorem [21] asserts that an $n$-vertex graph $G$ contains a $K_{r}$-factor, provided $r \mid n$ and $\delta(G) \geq$ $((r-1) / r) n$. Thus, this extremal example is sharp. (Note, though, that for many $r$-partite graphs $F$, a significantly lower minimum degree condition than that in Theorem 1.1 ensures an $F$-factor; see [29].)

As for many other problems in the area, this extremal example has the characteristic that it contains a large independent set. There has thus been significant interest in seeking variants of classical results in extremal graph theory, where one now forbids the host graph from containing a large independent set. Indeed, nearly 50 years ago, Erdős and Sós [18] initiated the study of the Turán problem under the additional assumption of a small independence number. That is, they considered the number of edges in an $n$-vertex $K_{r}$-free graph with independence number $o(n)$. This topic is now known as Ramsey-Turán theory and has been extensively studied by numerous authors (see, for example, [2, 17, 31, 34]). More 
recently, there has been interest in similar questions but where now one seeks a $K_{r}$-factor in an $n$-vertex graph with independence number $o(n)$ and large minimum degree (see [3, 4, 22]).

A stronger notion of a graph not containing a large independent set is that of being locally dense. More precisely, given $\rho, d>0$, we say that an $n$-vertex graph $G$ is $(\rho, d)$-dense if every $X \subseteq V(G)$ satisfies $e(G[X]) \geq d\left(\begin{array}{c}|X| \\ 2\end{array}\right)-\rho n^{2}$. Note that the property of being locally dense is weaker than being dense and (pseudo)random and stronger than having a sublinear independence number. Locally dense graphs have been considered in a number of previous papers. For example, there have been several papers on a question of Erdôs, Faudree, Rousseau, and Schelp [16]; there they considered a variant of the notion of $(\rho, d)$-dense and asked for the values of $\rho$ and $d$ guaranteeing that a $(\rho, d)$-dense graph contains a triangle. One can view the notion of locally dense as a parameter that ensures a graph is in some sense 'random-like'. Therefore, there has been interest in determining the number of (homomorphic) copies of a fixed graph $H$ in a $(\rho, d)$-dense graph $G$, and in particular whether this count is close to the value obtained if $G$ were a random graph; the study of this topic (for graphs and hypergraphs) was initiated by Kohayakawa, Nagle, Rödl, and Schacht [25].

The aim of this paper is to prove the following locally dense version of the bandwidth theorem.

Theorem 1.2. For all $\Delta \in \mathbb{N}$ and $d, \eta>0$, there exist constants $\rho, \beta, n_{0}>0$ such that for every $n \geq n_{0}$, the following holds. Let $H$ be an n-vertex graph with $\Delta(H) \leq \Delta$ and bandwidth at most $\beta n$. Then any $(\rho, d)$-dense graph $G$ on $n$ vertices with $\delta(G) \geq(1 / 2+\eta) n$ contains a copy of $H$.

In the case when $H$ corresponds to a $K_{r}$-factor, Theorem 1.2 had been proven by Reiher and Schacht (see [4]). Note that in the case when $H$ is connected, the minimum degree in Theorem 1.2 is bestpossible up to the $\eta n$ term. Indeed, if $G$ consists of two vertex-disjoint cliques, each of size $n / 2$, then $G$ trivially does not contain $H$, although $G$ is locally dense and $\delta(G)=n / 2-1$.

A striking feature of Theorem 1.2 is that, unlike Theorem 1.1, the minimum-degree condition does not depend on the chromatic number of $H$. In particular, when $\chi(H)=2$, the minimum-degree condition in Theorem 1.2 is the same as that in Theorem 1.1. Thus, in the case of bipartite $H$, there is no benefit in adding the condition that $G$ is locally dense. However, when $\chi(H)>2$, the minimum degree in Theorem 1.2 is substantially reduced compared to the bandwidth theorem.

It would also be extremely interesting to prove a version of Theorem 1.2 for graphs of sublinear independence number. Note, though, that examples in [4] show that the statement of Theorem 1.2 is far from true if we require that $G$ has a sublinear independence number instead of the locally dense condition. Indeed, the minimum degree necessary for the existence of a $K_{r}$-factor in such a graph is at least $\left(\frac{r-2}{r}+o(1)\right) n$ for every $r \geq 4$. So, these two problems are genuinely different.

The proof of Theorem 1.2 draws on ideas from [8,9], and our approach makes use of the regularityblow-up method. We also employ several new ideas (particularly with regard to dealing with so-called exceptional vertices). In the next section, we give an overview of the proof of Theorem 1.2. In Section 3, we introduce some notation as well as several fundamental properties of locally dense graphs. The regularity and blow-up lemmas are presented in Section 4. A key step in the proof of Theorem 1.2 is to show that the hypothesis of this theorem ensures that $G$ contains the $r$ th power of a Hamilton cycle; we prove this in Section 5. The proof of Theorem 1.2 then breaks into two main parts: the proof of two socalled lemmas for $H$ (presented in Section 6) and the lemma for $G$ (presented in Section 7). In Section 8, we combine all these results to prove Theorem 1.2. We give some concluding remarks in Section 9.

Additional note. Since this paper was first submitted, Ebsen, Maesaka, Reiher, Schacht, and Schülke [14] have built on our work to generalise Theorem 1.2. Indeed, they replace the minimum degree condition on $G$ with an inseparability condition.

\section{Overview of the proof of Theorem 1.2}

The overall strategy follows in the same spirit as the proof of the bandwidth theorem in [9], although the precise details of the proofs of the key steps in the argument turn out to be quite different. In particular, the setting of locally dense graphs both smooths over some aspects of the proof and introduces additional 
difficulties. Often, in problems involving embedding a spanning structure, the most challenging aspect of the proof is dealing with so-called exceptional vertices (that is, trying to cover either the remaining last few vertices in the host graph or those few vertices that do not fit in some general structure in the host graph). In this paper, we take a novel approach to dealing with such vertices. Below we outline the key steps in our proof and highlight some of the main novelties in our approach.

Obtaining structure in $G$. Suppose that $H$ and $G$ are as in the statement of the theorem where $\chi(H)=r$. The first step in the proof is to apply the regularity lemma (Lemma 4.1) to $G$ to obtain the reduced graph $R$ of $G$. The reduced graph $R$ is locally dense (with somewhat different parameters compared to $G)$ and 'inherits' the minimum degree of $G$ (that is, $\delta(R)>(1 / 2+o(1))|R|)$. These properties ensure that $R$ contains an almost spanning subgraph $Z_{\ell}^{2 r}$ that has the following properties:

- $Z_{\ell}^{2 r}$ covers all but at most $2 r$ of the vertices in $R$.

○ $Z_{\ell}^{2 r}$ consists of $\ell$ vertex-disjoint copies $K^{1}, \ldots, K^{\ell}$ of $K_{2 r}$ (in particular, $\left|Z_{\ell}^{2 r}\right|=2 r \ell$ ).

○ For each $1 \leq i \leq \ell$, there are all possible edges between $K^{i}$ and $K^{i+1}$ except that we miss a perfect matching between the two. (Note here that $K^{\ell+1}:=K^{1}$.)

The existence of $Z_{\ell}^{2 r}$ in $R$ can be guaranteed by finding a sufficiently large power of a Hamilton cycle in $R$. This is achieved in Section 5 (see Theorem 5.1). Using this, one can easily deduce that $G$ contains an almost spanning structure $\mathcal{C}$ that looks like the 'blow-up' of $Z_{\ell}^{2 r}$. More precisely, if $V\left(Z_{\ell}^{2 r}\right)=\{1, \ldots, 2 r \ell\}$ and $V_{1}, \ldots, V_{2 r \ell}$ are the corresponding clusters in $G$; then

(i) $V(\mathcal{C})=V_{1} \cup \cdots \cup V_{2 r \ell}$.

(ii) $\mathcal{C}\left[V_{i}, V_{j}\right]$ is $\varepsilon$-regular whenever $i j \in E\left(Z_{\ell}^{2 r}\right)$.

(iii) If $j k$ is an edge in one of the cliques $K^{i}$, then $\mathcal{C}\left[V_{j}, V_{k}\right]$ is superregular.

We refer to $\mathcal{C}$ as a cycle structure.

Suppose that in fact $\mathcal{C}$ is a spanning subgraph of $G$. In this case, ideally, one would now like to take the following approach. Let $x_{1}, \ldots, x_{n}$ denote the bandwidth ordering of $H$. Partition $V(H)$ into $\ell$ classes $C_{1}, \ldots, C_{\ell}$ so that

$\circ c_{i}:=\left|C_{i}\right|=\left|\cup_{j \in V\left(K^{i}\right)} V_{j}\right|$ for all $1 \leq i \leq \ell$.

○ $C_{1}$ contains the vertices $x_{1}, \ldots, x_{c_{1}}, C_{2}$ contains the vertices $x_{c_{1}+1}, \ldots, x_{c_{1}+c_{2}}$, and so forth.

Then embed the vertices from $C_{1}$ into the clusters in $G$ corresponding to the clique $K^{1}$, embed the vertices from $C_{2}$ into the clusters in $G$ corresponding to the clique $K^{2}$, and so on.

At first sight, this seems like a plausible strategy: since the partition of $V(H)$ respected the bandwidth ordering of $H$ (and as $H$ has small bandwidth), most edges in $H$ lie in the induced subgraphs $H\left[C_{i}\right]$; all remaining edges lie in the bipartite graphs $H\left[C_{i}, C_{i+1}\right]$. Suppose one could map each $C_{i}$ onto the clusters corresponding to $K^{i}$, so that each such cluster $V_{j}$ receives precisely $\left|V_{j}\right|$ vertices from $C_{i}$ and, crucially, all edges $x y$ in $H\left[C_{i}\right]$ are such that $x$ and $y$ are mapped to different clusters in $K^{i}$. That is, suppose we have a graph homomorphism $\phi_{i}$ between $H\left[C_{i}\right]$ and $K^{i}$ that maps precisely $\left|V_{j}\right|$ vertices to each $V_{j}$. Further, suppose the $\phi_{i}$ together combine to give a graph homomorphism $f$ from $H$ to $Z_{\ell}^{2 r}$ (so the edges in each $H\left[C_{i}, C_{i+1}\right]$ are mapped to edges in $\left.R\left[V\left(K^{i}\right), V\left(K^{i+1}\right)\right]\right)$. Set $G_{i}:=G\left[\cup_{j \in V\left(K^{i}\right)} V_{j}\right]$. Then (iii) above ensures that we could apply the blow-up lemma to each graph $G_{i}$ so as to embed $H$ [ $\left.C_{i}\right]$ into $G_{i}$. Further, (ii) ensures that we can achieve this embedding so all edges in the graphs $H\left[C_{i}, C_{i+1}\right]$ are also present. That is, we would obtain an embedding of $H$ into $G$.

This naive approach fails, though, as there is no guarantee one can map each $C_{i}$ onto the clusters corresponding to $K^{i}$ so that each such cluster $V_{j}$ receives precisely $\left|V_{j}\right|$ vertices from $C_{i}$. Furthermore, in the above approach, we assumed that $\mathcal{C}$ is a spanning subgraph of $G$; in reality, we have a small exceptional set $V_{0}$ of vertices in $G$ uncovered by $\mathcal{C}$.

The basic lemma for $H$ and the lemma for $G$. Instead of the above, we prove the so-called basic lemma for $H$ (Lemma 6.1). Here we show that one can find a graph homomorphism $f$ from $H$ into $Z_{\ell}^{2 r}$ so that for every cluster $V_{i}$ of $R$, approximately $\left|V_{i}\right|$ vertices are mapped to it. This therefore 'almost' 
gives us the desired graph homomorphism $f$ from $H$ into $Z_{\ell}^{2 r}$. In the proof of Lemma 6.1, we rely on the fact that the $K^{i}$ in $Z_{\ell}^{2 r}$ are copies of $K_{2 r}$; note that in the analogous structure in the proof of the bandwidth theorem [9], the $K^{i}$ are copies of $K_{r}$. To see why our condition is helpful for us, note that whilst in general, an $r$-partite graph $H^{\prime}$ does not have an 'almost balanced' graph homomorphism into $K_{r}$ (since $H^{\prime}$ may have colour classes of wildly different sizes), for $r$-partite graphs $H^{\prime}$ of bounded degree and sublinear bandwidth, one can always find an almost balanced graph homomorphism from $H^{\prime}$ into $K_{2 r}$.

Next, in the lemma for $G$ (Lemma 7.1), we prove that if one does not have an exceptional set $V_{0}$, then we can move vertices around the cycle structure $\mathcal{C}$ in such a way as to ensure that now each cluster $V_{i}$ in $\mathcal{C}$ has size precisely corresponding to the number of vertices mapped to $V_{i}$ by $f$. This is at the expense of weakening condition (iii): after applying Lemma 7.1, we only have that each clique $K^{i}$ splits into two cliques $K_{1}^{i}$ and $K_{2}^{i}$ of size $r$ such that if $j k$ is an edge in one of the cliques $K_{1}^{i}$ or $K_{2}^{i}$, then $\mathrm{C}\left[V_{j}, V_{k}\right]$ is superregular. However, this is still good enough to apply the blow-up lemma to find our desired embedding of $H$ into $G$.

Special lemma for $H$. So far, we have been assuming that there is no exceptional set $V_{0}$; further, in the the proof of the bandwidth theorem [9], Böttcher, Schacht, and Taraz were able to utilise the large minimum degree to incorporate exceptional vertices into (their analogue of the cycle structure) $\mathcal{C}$. We have a significantly smaller minimum degree and so are unable to do this in our setting.

Instead, given the bandwidth ordering $x_{1}, \ldots, x_{n}$ of $H$, we reserve a short initial segment $x_{1}, \ldots, x_{t}$; and let $H^{\prime}$ denote the subgraph of $H$ induced by $x_{1}, \ldots, x_{t}$. Here, $t$ will be significantly bigger than $\beta n$ (recall that $H$ has bandwidth at most $\beta n$ ), but $H^{\prime}$ will still only be a small fraction of $H$. Via the special lemma for $H$ (Lemma 6.2), we will embed $H^{\prime}$ into $G$ in such a way that, crucially, all of $V_{0}$ is covered by $H^{\prime}$ and, equally importantly, we do not cover more than a small proportion of each cluster $V_{i}$ in $\mathrm{C}$.

In the proof of Lemma 6.2, since $V_{0}$ may contain only very few (or even no) edges, we must assign an independent set $I$ in $H^{\prime}$ of size $\left|V_{0}\right|$ to be embedded onto $V_{0}$. We then must connect up $I$ through the rest of $G$ to obtain a copy of $H^{\prime}$. In particular, since $H^{\prime}$ is much smaller than $H$, the distance between two vertices $x, y \in I$ in $H^{\prime}$ may also be small. So it is crucial that $G$ is 'highly connected'. The connecting lemma (Lemma 3.3) ensures that this is the case. (Lemma 3.3 is also applied in the proof of Theorem 5.1.)

Care is also needed to ensure that Lemma 6.2 is compatible with the basic lemma for $H$ (Lemma 6.1). That is, we use Lemma 6.2 to embed $H^{\prime}$ in $G$ and Lemma 6.1 to embed $H \backslash H^{\prime}$ in $G$. Thus, we need to ensure that the copies of $H^{\prime}$ and $H \backslash H^{\prime}$ can be positioned in $G$ in such a way that they 'glue' together to form a copy of $H$.

Note that the reader should view the above overview as an idealisation of the proof. Indeed, when we prove Theorem 1.2 in Section 8, some of the details will be a little different. For example, for technical reasons, it is important that we find a spanning copy of $Z_{\ell}^{r^{*}}$ in $R$ for some $r^{*} \gg r$ rather than $Z_{\ell}^{2 r}$.

\section{Preliminaries}

\subsection{Notation}

Given a set $X$ and $k \leq|X|$, write $\left(\begin{array}{l}X \\ k\end{array}\right)$ for the set of $k$-element subsets of $X$. Given $r \in \mathbb{N}$, write $[[2 r]]^{2}:=[r]^{2} \cup([2 r] \backslash[r])^{2}$. Given a function $f: X \rightarrow Y$ and $A \subseteq X$, we write $\left.f\right|_{A}$ for the restriction of $f$ to $A$ and $f(A):=\{f(a): a \in A\}$.

Given a graph $G$, we write $V(G)$ and $E(G)$ for the vertex and edge sets respectively, and $|G|:=|V(G)|$ and $e(G):=|E(G)|$. The degree of a vertex $x \in V(G)$ is denoted by $d_{G}(x)$ and its neighbourhood by $N_{G}(x)$. The degree of a subset $X \subseteq V(G)$ is $d_{G}(X):=\left|\bigcap_{x \in X} N_{G}(x)\right|$. A subgraph $H \subseteq G$ is $s$ extendable if $d_{G}(V(H)) \geq s$. Given vertices $x_{1}, \ldots, x_{k}$, we write $N_{G}\left(x_{1}, \ldots, x_{k}\right):=\bigcap_{1 \leq i \leq k} N_{G}\left(x_{i}\right)$. If $A \subseteq V(G)$, we write $N_{G}(x, A):=N_{G}(x) \cap A$ and $d_{G}(x, A):=\left|N_{G}(x) \cap A\right|$. We say that $A$ is $k$ independent if every vertex in $A$ is at distance at least $k+1$ in $G$; that is, the shortest path in $G$ between any pair of elements in $A$ has length at least $k+1$. Given $X, Y \subseteq V(G)$ (not necessarily disjoint), define $e_{G}(X, Y)$ to be the number of edges $x y \in E(G)$ with $x \in X$ and $y \in Y$. If $X$ and $Y$ are disjoint, then 
$G[X, Y]$ is the bipartite graph with vertex classes $X$ and $Y$ whose edge set consists of all those edges in $G$ with one endpoint in $X$ and the other in $Y$.

Given two graphs $G$ and $H$, we say that $f: V(H) \rightarrow V(G)$ is a graph homomorphism if $f(x) f(y) \in$ $E(G)$ whenever $x y \in E(H)$. If $f$ is additionally injective, we say that $f$ is an embedding (of $H$ into $G$ ). Then $H \subseteq G$.

Throughout the paper, we will ignore floors and ceilings wherever they do not affect the argument. The constants in the hierarchies used to state our results are chosen from right to left. For example, if we claim that a result holds whenever $0<1 / n \ll a \ll b \ll c \leq 1$ (where $n$ is the order of the graph), then there are non-decreasing functions $f:(0,1] \rightarrow(0,1], g:(0,1] \rightarrow(0,1]$, and $h:(0,1] \rightarrow(0,1]$ such that the result holds for all $0<a, b, c \leq 1$ and all $n \in \mathbb{N}$ with $b \leq f(c), a \leq g(b)$, and $1 / n \leq h(a)$. Note that $a \ll b$ implies that we may assume in the proof that, for example, $a<b$ or $a<b^{2}$.

Given numbers $a, b, c$, we write $a=b \pm c$ to mean $a \in[b-c, b+c]$.

\subsubsection{Named graphs}

Given a graph $H$, the graph $H^{r}$, called the $r$ th power of $H$, is obtained from $H$ by adding an edge between every pair of vertices of distance at most $r$ in $H$. In particular:

○ $P_{k}^{r}=P=v_{1} \ldots v_{k}$ is an $r$-path if $V(P)=\left\{v_{1}, \ldots, v_{k}\right\}$ and $E(P)=\bigcup_{j \in[r]}\left\{v_{i} v_{i+j}: 1 \leq i \leq k-j\right\}$.

$\circ C_{k}^{r}=C=w_{1} \ldots w_{k}$ is an $r$-cycle if $V(C)=\left\{w_{1}, \ldots, w_{k}\right\}$ and $E(C)=\bigcup_{j \in[r]}\left\{w_{i} w_{i+j}: 1 \leq i \leq\right.$ $k\}$, where addition is modulo $k$.

Additionally,

$\circ F$ is an $r$-trail (of length $s$ ) if there exists an ordered sequence of not necessarily distinct vertices $v_{1}, \ldots, v_{s}$ such that $V(F)=\left\{v_{1}, \ldots, v_{s}\right\}$ and $E(F)=\bigcup_{j \in[r]}\left\{v_{i} v_{i+j}: 1 \leq i \leq s-j\right\}$. Observe that $P_{k}^{r}$ is an $r$-trail, and $F \cong P_{s}^{r}$ if and only if $|F|=s$.

- A $K$-tiling is a collection of vertex disjoint copies of $K$. If it contains $t$ copies, we denote it by $t \cdot K$. If $H \subseteq G$ is a $K$-tiling that is also spanning, we say that $H$ is a $K$-factor of $G$.

Define

○ $Z_{\ell}^{r}$ to be the graph with vertex set $[\ell] \times[r]$ in which $(i, j)\left(i^{\prime}, j^{\prime}\right)$ is an edge whenever $(\mathrm{i})\left|i-i^{\prime}\right| \leq 1$ and $j \neq j^{\prime}$ and when (ii) $i=\ell, i^{\prime}=1$, and $j \neq j^{\prime}$.

Thus, $Z_{\ell}^{r}$ is obtained from a cycle on $\ell$ vertices by replacing each vertex with a clique on $r$ vertices and replacing every edge with a complete bipartite graph minus a certain perfect matching. As indicated in Section 2, $Z_{\ell}^{2 r}$ will be used in the proof of Theorem 1.2 as a framework for embedding (most of) $H$ into $G$. Note that Böttcher, Schacht, and Taraz [9] used a very similar structure in their proof of the bandwidth theorem.

Observe that

$$
2 \ell \cdot K_{r} \subseteq C_{2 r \ell}^{r-1} \subseteq Z_{2 \ell}^{r} \subseteq C_{2 r \ell}^{2 r-1} \subseteq Z_{\ell}^{2 r}
$$

and the lexicographic ordering of $V\left(Z_{\ell}^{r}\right)$ (that is, $\left.(1,1)(1,2), \ldots,(1, r),(2,1), \ldots,(\ell, r)\right)$ is an $(r-1)$ cycle ordering of $C_{r \ell}^{r-1}$.

Given $A, B \subseteq V(G)$ and $x_{1}, \ldots, x_{k} \in V(G)$, when we say that, for example, $A B x_{1} \ldots x_{k}$ is an $r$-path (respectively, $r$-trail, $r$-cycle), we mean that any ordering $a_{1}, \ldots, a_{|A|}$ of $A$ and any ordering $b_{1}, \ldots, b_{|B|}$ of $B$ are such that $a_{1} \ldots a_{|A|} b_{1} \ldots b_{|B|} x_{1} \ldots x_{k}$ is an $r$-path (respectively, $r$-trail, $r$-cycle). An $r$-path (respectively, $r$-trail, $r$-cycle), $A x_{1} \ldots x_{k} B$ or $x_{1} \ldots x_{k} A B$ is defined analogously.

Suppose $X$ and $Y$ are disjoint sets of vertices of size $r$. We say that an $r$-path $P$ is between $X$ and $Y$ if $P=X x_{1} \ldots x_{k} Y$ for some vertices $x_{1}, \ldots, x_{k}$. Observe that $P[X], P[Y] \cong K_{r}$. Further, $P$ avoids a set $W \subseteq V(G)$ if $V(P) \cap W=\emptyset$. 


\subsection{Properties of locally dense graphs}

In this section we prove some simple properties of locally dense graphs $G$ : that $G$ induced on a large vertex subset is still locally dense; after removing a small set of vertices, $G$ is still locally dense; and $G$ contains many copies of cliques of a fixed size that additionally have a large common neighbourhood.

A fact that we shall use often throughout the paper is that if $0<\rho<\rho^{\prime}$ and $0<d^{\prime}<d$, then a $(\rho, d)$-dense graph is also $\left(\rho^{\prime}, d^{\prime}\right)$-dense.

Lemma 3.1. Let $r, n \in \mathbb{N}$ and $0<1 / n \ll \rho \ll d, 1 / r$, and $0<d, \alpha<1$. Let $G$ be a $(\rho, d)$-dense graph on $n$ vertices, and let $U \subseteq V(G)$ where $|U|=\alpha n$. Then

(i) $G[U]$ is $\left(\rho / \alpha^{2}, d\right)$-dense.

(ii) $G \backslash U$ is $\left(\rho /(1-\alpha)^{2}, d\right)$-dense.

(iii) $G$ contains at least dn/2 vertices of degree at least $d n / 2$.

(iv) $G$ contains at least $(d / 2)\left(\begin{array}{c}r+1 \\ 2\end{array}\right) n^{r} / r$ ! copies of $K_{r}$, each of which is $d^{r} n / 2^{r}$-extendable.

Proof. The proof of (i) is clear, and (ii) follows immediately from (i). For (iii), let $Y:=\{v \in V(G)$ : $\left.d_{G}(v) \geq d n / 2\right\}$. Then

$$
2 d\left(\begin{array}{l}
n \\
2
\end{array}\right)-2 \rho n^{2} \leq 2 e(G) \leq(n-|Y|) \frac{d n}{2}+|Y| n
$$

and so

$$
|Y| \geq \frac{d n-2 d-4 \rho n}{2-d} \geq \frac{d n}{2}
$$

proving (iii).

It remains to prove (iv). We claim that for each $i \leq r$, there is a set $\mathcal{T}_{i}$ of (ordered) tuples $\mathbf{x}=$ $\left(x_{1}, \ldots, x_{i}\right)$ such that $G\left[\left\{x_{1}, \ldots, x_{i}\right\}\right] \cong K_{i}$ and $d_{G}\left(\left\{x_{1}, \ldots, x_{i}\right\}\right) \geq d^{i} n / 2^{i}$ for all $\mathbf{x} \in \mathcal{T}_{i}$, and $\left|\mathcal{T}_{i}\right| \geq(d / 2)\left(\begin{array}{c}i+1 \\ 2\end{array}\right) n^{i}$. This will immediately imply (iv) as $\mathcal{T}_{r}$ gives rise to at least $(d / 2)\left(\begin{array}{c}r+1 \\ 2\end{array}\right) n^{r} / r$ ! (unlabelled) copies of $K_{r}$, each of which is $d^{r} n / 2^{r}$-extendable.

We will prove this by induction on $i$. Part (iii) implies that $G$ contains a set $\mathcal{T}_{1}$ of $d n / 2$ copies of $K_{1}$ that are all $d n / 2$-extendable. Suppose we have obtained $\mathcal{T}_{i-1}$ with the required properties for some $2 \leq i \leq r$.

Fix $\mathbf{x}=\left(x_{1}, \ldots, x_{i-1}\right) \in \mathcal{T}_{i-1}$. The graph $G_{\mathbf{x}}:=G\left[N_{G}\left(x_{1}, \ldots, x_{i-1}\right)\right]$ induced by its neighbourhood contains at least $d^{i-1} n / 2^{i-1}$ vertices, so (i) implies that it is $\left(2^{2 i-2} \rho / d^{2 i-2}, d\right)$-dense and hence $(\sqrt{\rho}, d)$ dense. Now, using the fact that $1 / n \ll \sqrt{\rho} \ll d, 1 / r$, (iii) implies that $G_{\mathbf{x}}$ contains at least $(d / 2)$. $d^{i-1} n / 2^{i-1}=d^{i} n / 2^{i}$ vertices, each of degree at least $d^{i} n / 2^{i}$. Each such vertex $y$ gives rise to an $r$-tuple $\mathbf{x}(y):=\left(x_{1}, \ldots, x_{i-1}, y\right)$. Certainly $G\left[\left\{x_{1}, \ldots, x_{i-1}, y\right\}\right] \cong K_{i}$; and further, $d_{G}\left(\left\{x_{1}, \ldots, x_{i-1}, y\right\}\right) \geq$ $d^{i} n / 2^{i}$ since $y$ has at least this many neighbours in the common neighbourhood of $\mathbf{x}$. Let $\mathcal{T}_{i}$ be the collection of all these tuples $\mathbf{x}(y)$ formed from each $\mathbf{x} \in \mathcal{T}_{i-1}$. Observe that they have the required properties and are all distinct, so

$$
\left|\mathcal{T}_{i}\right| \geq d^{i} n / 2^{i} \cdot\left|\mathcal{T}_{i-1}\right| \geq(d / 2)^{\left(\begin{array}{c}
i \\
2
\end{array}\right)+i} n^{i}=(d / 2)^{\left(\begin{array}{c}
i+1 \\
2
\end{array}\right)} n^{i}
$$

This completes the proof of the lemma.

We will need a connecting lemma to find a short $r$-path between two 'extendable' copies of $K_{r}$ in a locally dense graph $G$ with $\delta(G)>(1 / 2+o(1)) n$. The heart of the proof is the following lemma, which is the only part of the proof of Theorem 1.2 that requires $\delta(G)>(1 / 2+o(1)) n$ (elsewhere, linear minimum degree suffices). Somewhat similar lemmas have been used elsewhere in other settings: for example, [19, 35].

Lemma 3.2. Let $0<1 / n \ll \rho \ll d, \eta, 1 / r<1$, where $n, r \in \mathbb{N}$. Let $G$ be an $n$-vertex graph, and let $U \subseteq V(G)$ be a subset of size $n^{\prime} \geq \eta n / 2$ such that $G[U]$ is $(\rho, d)$-dense and $d_{G}(x, U) \geq(1 / 2+\eta) n^{\prime}$ 
for all $x \in V(G)$. Let $X, Y, W$ be pairwise disjoint subsets of $V(G)$ such that $|X|=|Y|=\lceil 4 r / \eta\rceil$ and $|W| \leq \eta n^{\prime} / 2$. Then there is $Z \subseteq U$ such that

(i) $G[Z] \cong K_{r}$.

(ii) $Z \cap(X \cup Y \cup W)=\emptyset$.

(iii) There exist $X^{\prime} \subseteq X$ and $Y^{\prime} \subseteq Y$ with $\left|X^{\prime}\right|=\left|Y^{\prime}\right|=r$ such that $N_{G}(Z) \supseteq X^{\prime} \cup Y^{\prime}$.

Proof. Let $C:=\lceil 4 r / \eta\rceil$, and let $U^{\prime}:=U \backslash(X \cup Y \cup W)$. Then

$$
e_{G}\left(U^{\prime}, X \cup Y\right) \geq(|X|+|Y|)\left((1 / 2+\eta) n^{\prime}-|X|-|Y|-|W|\right) \geq 2 C(1 / 2+\eta / 4) n^{\prime} \geq(1+\eta / 2) C n^{\prime} .
$$

Let $U^{\prime \prime}$ be the collection of those vertices in $U^{\prime}$ that each have at least $C+r$ neighbours in $X \cup Y$. Then $(1+\eta / 2) C n^{\prime} \leq(C+r-1)\left(n^{\prime}-\left|U^{\prime \prime}\right|\right)+2 C\left|U^{\prime \prime}\right|$, and so

$$
\left|U^{\prime \prime}\right| \geq \frac{(1+\eta / 2) C n^{\prime}-(C+r-1) n^{\prime}}{C-r+1} \geq \frac{\eta n^{\prime}}{4},
$$

where the final inequality follows from the fact that $C \geq 4 r / \eta$. There are not more than $2^{2 C}$ ways a vertex can attach to $X \cup Y$, so there is $U^{*} \subseteq U^{\prime \prime}$ such that $N_{G}(v, X \cup Y)$ is identical for all $v \in U^{*}$ and $\left|U^{*}\right| \geq \eta n^{\prime} / 2^{2 C+2}$. Note further that, since each such $v$ has at least $C+r$ neighbours in $X \cup Y$, there are $X^{\prime} \subseteq X$ and $Y^{\prime} \subseteq Y$ such that $\left|X^{\prime}\right|=\left|Y^{\prime}\right|=r$ and $X^{\prime} \cup Y^{\prime} \subseteq N_{G}\left(U^{*}\right)$. Lemma 3.1(i) now implies that $G\left[U^{*}\right]$ is $\left(2^{4 C+4} \rho / \eta^{2}, d\right)$-dense and hence $(\sqrt{\rho}, d)$-dense. But then, by Lemma 3.1(iv), there is $Z \subseteq U^{*}$ that spans a $K_{r}$. The desired properties (i)-(iii) are immediate.

As well as being applied in the proof of the connecting lemma below, Lemma 3.2 is also a key tool in the proof of Theorem 5.1 in Section 5, which in turn is a crucial tool for the proof of Theorem 1.2.

Lemma 3.3 (Connecting lemma). Let $0<1 / n \ll \rho \ll d, \eta \leq 1 / r$, where $r \in \mathbb{N}$, and let $G$ be $a$ $(\rho, d)$-dense graph on $n$ vertices with $\delta(G) \geq(1 / 2+\eta) n$. Let $W, X, Y$ be subsets of $V(G)$ such that $|W| \leq \eta n / 4$ and $X, Y$ induce $r$-cliques in $G$ and each one either

- lies in a copy of $K_{\lceil 9 r / \eta\rceil}$ that is disjoint from $W$ or

$\circ$ is $\eta n$-extendable.

Then $G$ contains a copy of $P_{3 r}^{r}=x_{1} \ldots x_{3 r}$ avoiding $W$ such that $X x_{1} \ldots x_{3 r}$ induces a copy of $P_{4 r}^{r}$, and $x_{1} \ldots x_{3 r} Y$ induces a copy of $P_{4 r}^{r}$.

The connecting lemma will ensure that the reduced graph $R$ of a graph $G$ (as in Theorem 1.2) is 'highly connected'. This property will be exploited when embedding a part of $H$ into $G$ so as to cover all of the exceptional set $V_{0}$ (specifically, we make use of Lemma 3.3 in Section 6.2).

Proof. Suppose that $X$ is $\eta n$-extendable. Let $C:=\lceil 9 r / \eta\rceil$ and $c:=\lceil 4 r / \eta\rceil$, and also let $G_{X}:=$ $G\left[N_{G}(X) \backslash W\right]$. Then Lemma 3.1(i) implies that $G_{X}$ is a $\left(16 \rho /\left(9 \eta^{2}\right), d\right)$-dense graph on at least $3 \eta n / 4$ vertices. But $4 /(3 \eta n) \ll 16 \rho /\left(9 \eta^{2}\right) \ll d, 1 / C$, so Lemma 3.1(iv) implies that $G_{X}$ contains a copy of $K_{C}$. Therefore, $X$ lies in a copy of $K_{C+r}$ that does not intersect $W$.

This implies that we may assume both $X, Y$ lie in a copy of $K_{C}$ that does not intersect $W$. Let $X^{*}$ be the vertex set of the $K_{C}$ containing $X$, and define $Y^{*}$ analogously for $Y$. Choose $X^{\prime} \subseteq X^{*}$ of size $c$ that is disjoint from $X$. Since $\left|Y^{*}\right|-|Y|-|X|-\left|X^{\prime}\right|=C-2 r-c \geq c$, we can choose $Y^{\prime} \subseteq Y^{*}$ of size $c$ that is disjoint from $X, Y, X^{\prime}$. Apply Lemma 3.2 with $n, r, \eta, V(G), X^{\prime}, Y^{\prime}, X \cup Y \cup W$ playing the roles of $n, r, \eta, U, X, Y, W$ to obtain $Z \subseteq V(G)$ that induces a copy of $K_{r}$ and is disjoint from $X^{\prime} \cup Y^{\prime} \cup X \cup Y \cup W$; and there exist $X^{\prime \prime} \subseteq X^{\prime}$ and $Y^{\prime \prime} \subseteq Y^{\prime}$ such that $\left|X^{\prime \prime}\right|=\left|Y^{\prime \prime}\right|=r$ and $X^{\prime \prime} \cup Y^{\prime \prime} \subseteq N_{G}(Z)$. Notice that, by construction, each of $X \cup X^{\prime \prime}, X^{\prime \prime} \cup Z, Z \cup Y^{\prime \prime}$, and $Y^{\prime \prime} \cup Y$ induce cliques, and the overlap of each consecutive pair induces a clique of size at least $r$. Further, none of these sets intersect with $W$. Thus $X X^{\prime \prime} Z Y^{\prime \prime} Y$ induces an $r$-path. Thus there is an $r$-path with vertex set $X^{\prime \prime} \cup Z \cup Y^{\prime \prime}$ (of length $3 r$ ) that has the required property. 


\section{The regularity and blow-up lemmas and associated tools}

\subsection{Regularity}

We will apply Szemerédi's regularity lemma in the proof of Theorem 1.2. For this, we need the following definitions. Given a bipartite graph $G$ with vertex classes $A$ and $B$ and parameters $\varepsilon, \delta \in(0,1)$,

$\circ$ let $d_{G}(A, B):=\frac{e_{G}(A, B)}{|A||B|}$ be the density of $G$, and say that $G$ is

$\circ \varepsilon$-regular if, for every $X \subseteq A$ and $Y \subseteq B$ with $|X| \geq \varepsilon|A|$ and $|Y| \geq \varepsilon|B|$, we have that $\left|d_{G}(A, B)-d_{G}(X, Y)\right| \leq \varepsilon$

$\circ(\varepsilon, \delta)$-regular if $G$ is $\varepsilon$-regular and additionally $d_{G}(A, B) \geq \delta$;

$\circ(\varepsilon, \delta)$-superregular if $G$ is $(\varepsilon, \delta)$-regular and additionally $d_{G}(a, B) \geq \delta|B|$ for every $a \in A$ and $d_{G}(b, A) \geq \delta|A|$ for every $b \in B$.

It will be convenient to use the degree form of the regularity lemma; this can be derived from the standard version [37].

Lemma 4.1 (Degree form of the regularity lemma). For all $\varepsilon \in(0,1)$ and $M^{\prime} \in \mathbb{N}$, there exist $M, n_{0} \in \mathbb{N}$ such that the following holds for all graphs $G$ on $n \geq n_{0}$ vertices and $\delta \in(0,1)$. There is a partition $V(G)=V_{0} \cup V_{1} \cup \ldots \cup V_{L}$ and a spanning subgraph $G^{\prime} \subseteq G$ such that

(i) $M^{\prime} \leq L \leq M$.

(ii) $\left|V_{0}\right| \leq \varepsilon n$.

(iii) $\left|V_{1}\right|=\ldots=\left|V_{L}\right|=: m$.

(iv) $d_{G^{\prime}}(x) \geq d_{G}(x)-(\delta+\varepsilon) n$ for all $x \in V(G)$.

(v) For all $i \in[L]$, the graph $G^{\prime}\left[V_{i}\right]$ is empty.

(vi) For all $i \in[L]$, the graph $G^{\prime}\left[V_{i}, V_{j}\right]$ is either empty or $(\varepsilon, \delta)$-regular.

We call $V_{1}, \ldots, V_{L}$ the clusters of $G$ and the vertices in $V_{0}$ the exceptional vertices. The graph $G^{\prime}$ is the pure graph. Note that the $(\varepsilon, \delta)$-regular pairs may have very different densities. The reduced graph $R$ of $G$ with parameters $\varepsilon, \delta$, and $M^{\prime}$ has vertex set $[L]$ and contains $i j$ as an edge precisely when $G^{\prime}\left[V_{i}, V_{j}\right]$ is $(\varepsilon, \delta)$-regular.

The next lemma states that the reduced graph $R$ of a locally dense graph $G$ is still locally dense (with worse parameters) and, further, $R$ inherits the minimum degree of $G$.

Lemma 4.2. Let $0<1 / n \ll 1 / M^{\prime} \ll \varepsilon \ll \delta \ll d \leq 1 ; 1 / M^{\prime} \ll \rho \ll d ; \delta \ll \eta$. Define $\rho^{*}:=$ $\max \{3 \rho, 3 \delta\}$. Let $G$ be a $(\rho, d)$-dense graph of order $n$ with $\delta(G) \geq(1 / 2+\eta) n$. Apply Lemma 4.1 with parameters $\varepsilon, \delta$, and $M^{\prime}$ to obtain a pure graph $G^{\prime}$ and a reduced graph $R$ of $G$ with $V(R)=[L]$. Then $R$ is $\left(\rho^{*}, d\right)$-dense with $\delta(R) \geq(1 / 2+\eta / 2) L$.

Proof. Here, (i)-(vi) will refer to the conclusions of Lemma 4.1. Parts (ii) and (iii) imply that

$$
(1-\varepsilon) n \leq m L \leq n
$$

Let $X \subseteq[L]$, and let $Y:=\bigcup_{i \in X} V_{i} \subseteq V(G)$. So $|Y|=m|X|$. Then

$$
d\left(\begin{array}{c}
|Y| \\
2
\end{array}\right)-\rho n^{2}-|Y|(\delta+\varepsilon) n \leq e(G[Y])-|Y|(\delta+\varepsilon) n \stackrel{(i v)}{\leq} e\left(G^{\prime}[Y]\right) \stackrel{(v)}{\leq} e(R[X]) \cdot m^{2}
$$

and so, dividing by $m^{2}$,

$$
e(R[X]) \stackrel{(4.1)}{\geq} d \cdot \frac{|X|^{2}-\frac{|X|}{m}}{2}-\rho\left(\frac{L}{1-\varepsilon}\right)^{2}-|X|(\delta+\varepsilon) \frac{L}{1-\varepsilon} \geq d\left(\begin{array}{c}
|X| \\
2
\end{array}\right)-\rho^{*} L^{2},
$$

as required. 
Let $i \in[L]$ and $x_{i} \in V_{i}$. Then $d_{G^{\prime}}\left(x_{i}\right) \geq d_{G}\left(x_{i}\right)-(\delta+\varepsilon) n \geq(1 / 2+\eta-\delta-\varepsilon) n$ by (iv). The number of clusters $V_{k}$ of $G$ containing some $y \in N_{G^{\prime}}\left(x_{i}\right)$ is therefore at least

$$
\frac{(1 / 2+\eta-\delta-\varepsilon) n-\left|V_{0}\right|}{m} \geq \frac{(1 / 2+\eta / 2) n}{m} \geq(1 / 2+\eta / 2) L
$$

But then (vi) implies that $i$ is adjacent to each of the vertices corresponding to these clusters in $R$. So $d_{R}(i) \geq(1 / 2+\eta / 2) L$, as required.

Note that in the case when $\rho \ll \delta$ in Lemma 4.2, $R$ only inherits the property being locally dense with a significantly worse parameter playing the role of $\rho$. That is, now $R$ is $(3 \delta, d)$-dense rather than $(\rho, d)$-dense.

The next well-known proposition states that (super)regular pairs are robust in the sense of adding or removing a small number of vertices. This version appears as Proposition 8 in [8].

Proposition 4.3. Let $G$ be a graph with $A, B \subseteq V(G)$ disjoint. Suppose that $G[A, B]$ is $(\varepsilon, \delta)$-regular, and let $A^{\prime}, B^{\prime} \subseteq V(G)$ be disjoint such that $\left|A \triangle A^{\prime}\right| \leq \alpha\left|A^{\prime}\right|$ and $\left|B \triangle B^{\prime}\right| \leq \alpha\left|B^{\prime}\right|$ for some $0 \leq \alpha<1$. Then $G\left[A^{\prime}, B^{\prime}\right]$ is $\left(\varepsilon^{\prime}, \delta^{\prime}\right)$-regular, with

$$
\varepsilon^{\prime}:=\varepsilon+6 \sqrt{\alpha} \text { and } \delta^{\prime}:=\delta-4 \alpha .
$$

If, moreover, $G[A, B]$ is $(\varepsilon, \delta)$-superregular and each vertex $x \in A^{\prime}$ has at least $\delta^{\prime}\left|B^{\prime}\right|$ neighbours in $B^{\prime}$ and each vertex $x \in B^{\prime}$ has at least $\delta^{\prime}\left|A^{\prime}\right|$ neighbours in $A^{\prime}$, then $G\left[A^{\prime}, B^{\prime}\right]$ is $\left(\varepsilon^{\prime}, \delta^{\prime}\right)$-superregular with $\varepsilon^{\prime}$ and $\delta^{\prime}$ as above.

The following lemma is well known in several variations. The version here follows immediately from [36, Lemma 4.6].

Lemma 4.4. Let $L \in \mathbb{N}$, and suppose that $0<1 / m \ll \varepsilon \ll \delta, 1 / \Delta, 1 / L \leq 1$. Let $R$ be a graph with $V(R)=[L]$ and $\Delta(R) \leq \Delta$. Let $G$ be a graph with vertex partition $V_{1}, \ldots, V_{L}$ such that $\left|V_{i}\right|=m$ for all $1 \leq i \leq L$ and in which $G\left[V_{i}, V_{j}\right]$ is $(\varepsilon, \delta)$-regular whenever $i j \in E(R)$. Then for each $i \in V(R)$, $V_{i}$ contains a subset $V_{i}^{\prime}$ of size $(1-\sqrt{\varepsilon}) m$ such that for every edge ij of $R$, the graph $G\left[V_{i}^{\prime}, V_{j}^{\prime}\right]$ is $(4 \sqrt{\varepsilon}, \delta / 2)$-superregular.

\subsection{Embedding lemmas}

The next lemma is similar to a partial embedding lemma from [8, Lemma 10], which in turn is similar to an embedding lemma due to Chvátal, Rödl, Szemerédi, and Trotter [11]. Given a homomorphism from a graph $H$ into the reduced graph $R$ of $G$ such that every pre-image is small, the lemma yields an embedding of some vertices of $H$ into $G$ while finding large candidate sets for the remaining vertices. Further (deviating from [8]), we would like to ensure that certain vertices of $H$ are embedded into given target sets of large size.

Lemma 4.5 (Embedding lemma with target sets). Let $0<1 / n \ll 1 / L \ll \varepsilon \ll c \ll \delta \ll 1 / \Delta$, where $n, L \in \mathbb{N}$. Let $G$ be an $n$-vertex graph, $R$ an $L$-vertex graph, and $H$ a graph on at most $\varepsilon n$ vertices such that

$\circ G$ has partition $\left\{V_{a}: a \in V(R)\right\}$, where $\left|V_{a}\right| \geq m \geq(1-\varepsilon) n / L$ for all $a \in V(R)$ and $G\left[V_{a}, V_{a^{\prime}}\right]$ is $(\varepsilon, \delta)$-regular whenever a $a^{\prime} \in E(R)$.

$\circ \Delta(H) \leq \Delta$, and there is a graph homomorphism $\phi: V(H) \rightarrow V(R)$ such that $\left|\phi^{-1}(a)\right| \leq 2 \varepsilon m$ for all $a \in V(R)$.

- Let $X \cup Y$ be a partition of $V(H)$, and suppose that there is $W \subseteq X$ such that for each $w \in W$, there is a set $S_{w} \subseteq V_{\phi(w)}$ with $\left|S_{w}\right| \geq \mathrm{cm}$. 
Then there is an embedding $f$ of $H[X]$ into $G$ such that

(i) $f(x) \in V_{\phi(x)}$ for all $x \in X$.

(ii) $f(w) \in S_{w}$ for all $w \in W$.

(iii) For all $y \in Y$, there exists $C_{y} \subseteq V_{\phi(y)} \backslash f(X)$ such that $C_{y} \subseteq N_{G}(f(x))$ for all $x \in N_{H}(y) \cap X$, and $\left|C_{y}\right| \geq \mathrm{cm}$.

Since the proof of Lemma 4.5 is essentially identical to that of Lemma 10 from [8], we omit the proof.

We will also use the blow-up lemma of Komlós, Sárközy, and Szemerédi [27], which states that, for the purposes of embedding a spanning $k$-partite graph $H$ of bounded degree, a graph $G$ with a vertex partition into $k$ classes, each pair of which is superregular, in fact behaves like a complete $k$-partite graph. Further, as in Lemma 4.5, one can ensure that a small fraction of the vertices of $H$ are embedded into some given target sets.

Lemma 4.6 (Blow-up lemma [27]). For every $d, \Delta, c>0$ and $k \in \mathbb{N}$, there exist constants $\varepsilon_{0}$ and $\alpha$ such that the following holds. Let $n_{1}, \ldots, n_{k}$ be positive integers, $0<\varepsilon<\varepsilon_{0}$, and $G$ be a $k$-partite graph with vertex classes $V_{1}, \ldots, V_{k}$ where $\left|V_{i}\right|=n_{i}$ for $i \in[k]$. Let $J$ be a graph on vertex set $[k]$ such that $G\left[V_{i}, V_{j}\right]$ is $(\varepsilon, d)$-superregular whenever $i j \in E(J)$. Suppose that $H$ is a $k$-partite graph with vertex classes $W_{1}, \ldots, W_{k}$ of size at most $n_{1}, \ldots, n_{k}$, respectively, with $\Delta(H) \leq \Delta$. Suppose further that there exists a graph homomorphism $\phi: V(H) \rightarrow V(J)$ such that $\left|\phi^{-1}(i)\right| \leq n_{i}$ for every $i \in[k]$. Moreover, suppose that in each class $W_{i}$, there is a set of at most $\alpha n_{i}$ special vertices $y$, each equipped with a set $S_{y} \subseteq V_{i}$ with $\left|S_{y}\right| \geq c n_{i}$. Then there is an embedding of $H$ into $G$ such that every special vertex $y$ is mapped to a vertex in $S_{y}$.

\section{Finding the power of a Hamilton cycle}

The next result states that for every $r \in \mathbb{N}$, every large locally dense $n$-vertex graph $G$ with minimum degree at least $(1 / 2+o(1)) n$ contains the $r$ th power of a Hamilton cycle. This is a very special case of our main result, Theorem 1.2.

Theorem 5.1. For all $r, s \in \mathbb{N}$ and $d, \eta>0$, there exist $\rho, n_{0}>0$ such that every $(\rho, d)$-dense graph $G$ on $n \geq n_{0}$ vertices with $\delta(G) \geq(1 / 2+\eta) n$ contains the rth power of a Hamilton cycle. In fact, for every $n^{\prime} \in \mathbb{N}$ such that $n-s \leq n^{\prime} \leq n, G$ contains the rth power of a cycle covering precisely $n^{\prime}$ vertices.

Note that Theorem 5.1 is an important tool in the proof of Theorem 1.2, in the same way that (an approximate version of) the result in [28] was used in the proof of Theorem 1.1. Indeed, Theorem 5.1 ensures that the reduced graph $R$ of a graph $G$ (as in Theorem 1.2) will contain a spanning $(4 r-1)$ cycle. By (3.1), this implies $R$ contains a spanning copy of $Z_{\ell}^{2 r}$. As outlined in Section 2, this copy of $Z_{\ell}^{2 r}$ will be used as a 'guide' for embedding $H$ into $G$.

We remark that one can give a significantly shorter proof of Theorem 5.1 if one only seeks the $r$ th power of a cycle covering (say) at least $(1-\eta) n$ vertices in $G$. However, for our application to Theorem 1.2, we (rather subtly) require that we have a $(4 r-1)$-cycle in $R$ covering all but a very small number of vertices (much fewer than $\rho|R|$ vertices in $R$ can be left uncovered). So, such a weaker version of Theorem 5.1 is not sufficient.

The proof of Theorem 5.1 is an application of the connecting-absorbing method, a technique first developed by Rödl, Ruciński, and Szemerédi [32]. The first step in the proof is to find a short absorbing $2 r$-path $P_{a b s}$ in $G$ that has the property that $V\left(P_{a b s}\right) \cup Z$ spans an $r$-path in $G$ (with the same startand endpoints as $P_{a b s}$ ) for any very small set of vertices $Z$. We then reserve a small pot of vertices $V^{\prime}$ (known as a reservoir), which will allow us to connect up pairs of paths into longer paths. Next we (via an application of the regularity lemma) find a collection $\mathcal{P}$ of a constant number of vertex-disjoint $2 C$ paths that together cover almost all of the remaining vertices in $G$ (here, $C$ is chosen to be significantly bigger than $r$ ). Using vertices from the reservoir, we are then able to connect all the paths in $\mathcal{P}$ together with $P_{a b s}$ to form a single $r$-cycle covering almost all the vertices in $G$. The remaining uncovered vertices in $G$ are absorbed by $P_{a b s}$ to obtain the $r$ th power of a Hamilton cycle. 
Proof of Theorem 5.1. Note that if $n$ is sufficiently large, then any $n^{\prime}$-vertex induced subgraph $G^{\prime}$ of an $n$-vertex graph $G$ as in the theorem must be $(2 \rho, d)$-dense with $\delta\left(G^{\prime}\right) \geq(1 / 2+\eta / 2) n^{\prime}$. So as the $r$ th power of a Hamilton cycle in $G^{\prime}$ corresponds to an $r$-cycle of length $n^{\prime}$ in $G$, it suffices to prove the first part of the statement of the theorem.

Further, it suffices to prove the theorem under the additional assumption that $d \ll \eta, 1 / r$. Define constants $\rho, \varepsilon, \delta, d_{1}, \eta_{0}, \eta_{1}, \eta_{2}, \eta_{3}>0$, and $M^{\prime} \in \mathbb{N}$, and apply the regularity lemma (Lemma 4.1) with inputs $\varepsilon$ and $M^{\prime}$ to obtain some $M=M\left(\varepsilon, M^{\prime}\right)$ so that we have

$$
0<1 / M \leq 1 / M^{\prime} \ll \varepsilon \ll \delta \ll \rho \ll \eta_{3} \ll \eta_{2} \ll \eta_{1} \ll \eta_{0} \ll d_{1} \ll d \ll \eta, 1 / r .
$$

Let $n$ be sufficiently large, and consider any $n$-vertex graph $G$ that is $(\rho, d)$-dense with $\delta(G) \geq(1 / 2+\eta) n$.

Our initial aim is to construct a small absorbing $2 r$-path $P_{a b s}$. The next claim provides the building blocks for this absorbing path.

Claim 5.2. There exists a collection $\mathcal{K}$ of at most $\eta_{0} n / 8 r$ vertex-disjoint copies of $K_{2 r}$ in $G$ such that:

(i) Each $K \in \mathcal{K}$ is $d_{1} n$-extendable in $G$.

(ii) Given any vertex $x \in V(G)$, there are at least $2 \eta_{2} n$ copies $K$ of $K_{2 r}$ in $\mathcal{K}$ so that $V(K) \subseteq N_{G}(x)$.

Proof (of claim). Let $\mathcal{C}$ denote the set of all copies of $K_{2 r}$ that are $d_{1} n$-extendable in $G$. So, certainly, $|\mathfrak{C}| \leq n^{2 r}$. Consider any $x \in V(G)$. Since $d_{G}(x) \geq n / 2$, Lemma 3.1(i) implies that $G[N(x)]$ is $(4 \rho, d)$ dense. Thus, Lemma 3.1(iv) implies that there are at least $(d / 2)\left(\begin{array}{c}2 r+1 \\ 2\end{array}\right)(n / 2)^{2 r} /(2 r)$ ! copies $K$ of $K_{2 r}$ in $\mathcal{C}$ so that $V(K) \subseteq N_{G}(x)$. (Here we use the property that $d^{2 r} / 2^{2 r} \geq d_{1}$ by (5.1).) Let $L_{x}$ denote the set of these copies of $K_{2 r}$.

Let $\mathcal{C}_{p}$ be obtained from $\mathcal{C}$ by selecting each $K \in \mathcal{C}$ independently with probability

$$
p:=\frac{\eta_{1}}{n^{2 r-1}} .
$$

Hence,

$$
\mathbb{E}\left(\left|\mathcal{C}_{p}\right|\right) \leq \eta_{1} n \text { and } \mathbb{E}\left(\left|\mathcal{C}_{p} \cap L_{x}\right|\right) \geq(d / 2)\left(\begin{array}{c}
(2 r+1 \\
2
\end{array}\right) \frac{(n / 2)^{2 r}}{(2 r) !} \times \frac{\eta_{1}}{n^{2 r-1}} \stackrel{(5.1)}{\geq} 2 d_{1} \eta_{1} n
$$

for each $x \in V(G)$. Thus, a Chernoff bound implies that, with high probability,

$$
\left|\mathcal{C}_{p}\right| \leq 2 \eta_{1} n \text { and }\left|\mathcal{C}_{p} \cap L_{x}\right| \geq d_{1} \eta_{1} n
$$

for all $x \in V(G)$. Let $Y$ denote the number of pairs of copies of $K_{2 r}$ from $\mathcal{C}_{p}$ that share at least one vertex. Then

$$
\mathbb{E}(Y) \leq p^{2}\left(\begin{array}{c}
n \\
2 r
\end{array}\right) 2 r\left(\begin{array}{c}
n \\
2 r-1
\end{array}\right) \leq \eta_{1}^{2} n .
$$

By Markov's inequality, the probability that $|Y| \leq 2 \eta_{1}^{2} n$ is at least $1 / 2$. Therefore, there is a choice of $\mathcal{C}_{p}$ such that this condition holds together with (5.2). Fix such a choice of $\mathcal{C}_{p}$; then for each intersecting pair of cliques in $\mathcal{C}_{p}$, remove one of them to obtain a new collection $\mathcal{K}$. Note that the definition of $\mathcal{C}_{p}$ and (5.2) implies that $\mathcal{K}$ is a collection of at most $\eta_{0} n / 8 r$ vertex-disjoint copies of $K_{2 r}$ in $G$. Further, since $d_{1} \eta_{1} n-2 \eta_{1}^{2} n \geq d_{1} \eta_{1} n / 2 \geq 2 \eta_{2} n$, we see that (ii) is satisfied, as desired.

With Claim 5.2 at hand, it is straightforward to obtain our desired absorbing $2 r$-path $P_{a b s}$.

Claim 5.3. $G$ contains a $2 r$-path $P_{a b s}$ on at most $\eta_{0} n$ vertices such that the following conditions hold.

(i) Both the sets of the first and last $2 r$ vertices on $P_{a b s}$ induce $K_{2 r} s$ in $G$ that are $d_{1} n$-extendable. (Denote these sets by $S$ and $E$, respectively.)

(ii) Given any set $Z \subseteq V(G) \backslash V\left(P_{a b s}\right)$ of size at most $\eta_{2} n$, there is an r-path $P$ in $G$ with vertex set $V\left(P_{a b s}\right) \cup Z$ whose first $2 r$ vertices are the elements of $S$ (ordered as in $P_{a b s}$ ) and last $2 r$ vertices are the elements of $E$ (ordered as in $P_{a b s}$ ). 
Proof (of claim). Let $\mathcal{K}$ be as in Claim 5.2, and enumerate its elements by $K^{1}, \ldots, K^{t}$ (so $t \leq \eta_{0} n / 8 r$ ). Apply Lemma 3.3 to $G$ with $d_{1}, 2 r, V\left(K^{1}\right), V\left(K^{2}\right), V(\mathcal{K})$ playing the roles of $\eta, r, X, Y, W$. (Note we can indeed apply this lemma by Claim 5.2(i) and as $|V(\mathcal{K})| \leq d_{1} n / 4$.) We thus obtain a copy $P_{1}=x_{1}^{1} \ldots x_{6 r}^{1}$ of $P_{6 r}^{2 r}$ in $G$, avoiding $V(\mathcal{K})$ such that $V\left(K^{1}\right) x_{1}^{1} \ldots x_{6 r}^{1}$ and $x_{1}^{1} \ldots x_{6 r}^{1} V\left(K^{2}\right)$ both induce copies of $P_{8 r}^{2 r}$. Repeating this process iteratively, we obtain a collection $P_{1}, \ldots, P_{t-1}$ of vertex-disjoint copies of $P_{6 r}^{2 r}$ in $G$ so that $V\left(K^{i}\right) x_{1}^{i} \ldots x_{6 r}^{i} V\left(K^{i+1}\right)$ induces a copy of $P_{8 r}^{2 r}$ in $G$ for each $1 \leq i \leq t-1$. (Here we have written $P_{i}=x_{1}^{i} \ldots x_{6 r}^{i}$.) Note that to ensure the $P_{i}$ s are vertex-disjoint, at every step we update $W$; so at step $i, W$ contains $V(\mathcal{K})$ and the vertices from $P_{1}, \ldots, P_{i-1}$ (so $|W| \leq d_{1} n / 4$ ).

Let $P_{a b s}$ denote the $2 r$-path obtained by the following concatenation:

$$
P_{a b s}:=V\left(K^{1}\right) P_{1} V\left(K^{2}\right) P_{2} V\left(K^{3}\right) \ldots V\left(K^{t-1}\right) P_{t-1} V\left(K^{t}\right) .
$$

Notice that $P_{a b s}$ contains $(t-1) 8 r+2 r \leq 8 r t \leq \eta_{0} n$ vertices. Further, (i) follows since both $K^{1}$ and $K^{t}$ are $d_{1} n$-extendable in $G$ by definition of $\mathcal{K}$. Consider any set $Z=\left\{z_{1}, \ldots, z_{\ell}\right\} \subseteq V(G) \backslash V\left(P_{a b s}\right)$ of size at most $\eta_{2} n$. For each $1 \leq i \leq \ell$, by Claim 5.2(ii), there are at least $\eta_{2} n$ choices for $j_{i}$ such that:

$\circ 2 \leq j_{i} \leq t-1$.

○ $V\left(K^{j_{i}}\right) \subseteq N_{G}\left(z_{i}\right)$.

In particular, writing $V\left(K^{j_{i}}\right)=\left\{y_{1}, \ldots, y_{2 r}\right\}$, notice that

$$
P_{j_{i}-1} y_{1} \ldots y_{r} z_{i} y_{r+1} \ldots y_{2 r} P_{j_{i}}
$$

is an $r$-path in $G$.

Since we have at least $\eta_{2} n$ choices, we may define $j_{1}, j_{2}, \ldots, j_{\ell}$ to be distinct. We can then insert each $z_{i}$ into $P_{a b s}$, as indicated by (5.3), to obtain the desired $r$-path $P$ on $V\left(P_{a b s}\right) \cup Z$.

Let $S$ be as in Claim 5.3. Then $\left|N_{G}(S) \backslash V\left(P_{a b s}\right)\right| \geq d_{1} n / 2$. Lemma 3.1(i) implies that $G_{S}:=$ $G\left[N_{G}(S) \backslash V\left(P_{a b s}\right)\right]$ is $\left(4 \rho / d_{1}^{2}, d\right)$-dense and therefore $\left(\rho^{1 / 2}, d\right)$-dense. Set

$$
C:=\left\lceil 4 r / \eta_{3}\right\rceil
$$

Note that $\rho^{1 / 2} \ll d, 1 / C$. Thus, Lemma 3.1(iv) implies that $G_{S}$ contains a copy $K_{2 C+1}^{S}$ of $K_{2 C+1}$. Similarly, we find a copy $K_{2 C+1}^{E}$ of $K_{2 C+1}$ in $G$ that is disjoint from $K_{2 C+1}^{S}$ and $P_{a b s}$ so that $V\left(K_{2 C+1}^{E}\right) \subseteq$ $N_{G}(E)$. We will view both $K_{2 C+1}^{S}$ and $K_{2 C+1}^{E}$ as $2 C$-paths of length $2 C+1$.

Set $G_{0}:=G \backslash\left(V\left(P_{a b s}\right) \cup V\left(K_{2 C+1}^{S}\right) \cup V\left(K_{2 C+1}^{E}\right)\right)$. Certainly, $\left|G_{0}\right| \geq\left(1-2 \eta_{0}\right) n$ and

$$
d_{G}\left(x, V\left(G_{0}\right)\right) \geq(1 / 2+3 \eta / 4) n \text { for all } x \in V(G) .
$$

By selecting vertices randomly (and applying a Chernoff bound), one can obtain a set $V^{\prime} \subseteq V\left(G_{0}\right)$ of $n^{\prime}:=\eta_{3} n$ vertices such that

$$
d_{G}\left(x, V^{\prime}\right) \geq(1 / 2+\eta / 2) n^{\prime} \text { for all } x \in V(G) .
$$

Set $G_{1}:=G\left[V^{\prime}\right]$ and $G_{2}:=G_{0} \backslash V^{\prime}$. Lemma 3.1(i) implies that $G_{1}$ is $\left(\rho / \eta_{3}^{2}, d\right)$-dense and thus $\left(\rho^{1 / 2}, d\right)$-dense. Similarly, $G_{2}$ is $(2 \rho, d)$-dense.

Apply Lemma 4.1 to $G_{2}$ with parameters $\varepsilon, \delta$, and $M^{\prime}$ to obtain a partition $V_{0}, V_{1}, \ldots, V_{\ell}$ of $V\left(G_{2}\right)$, pure graph $G_{2}^{\prime}$, and the reduced graph $R$ of $G_{2}$. Here, $V_{0}$ is the exceptional set on at most $\varepsilon n$ vertices, and $M^{\prime} \leq \ell \leq M$. Set $m:=\left|V_{1}\right|=\cdots=\left|V_{\ell}\right|$. Then Lemma 4.2 implies that $R$ is $(6 \rho, d)$-dense. In particular, Lemma 3.1(i) implies that $R^{\prime}$ is $\left(6 \rho / \eta_{3}^{2}, d\right)$-dense for any $R^{\prime} \subseteq R$ on $\eta_{3} \ell$ vertices.

Note that $1 / \ell \ll 6 \rho / \eta_{3}^{2} \ll d, 1 / C$. Thus, Lemma 3.1(iv) implies that every $R^{\prime} \subseteq R$ on $\eta_{3} \ell$ vertices contains a copy of $K_{2 C+1}$. In particular, $R$ contains a $K_{2 C+1}$-tiling $\mathcal{T}$ covering all but at most $\eta_{3} \ell$ vertices. 
Consider any copy $K$ of $K_{2 C+1}$ in $\mathcal{T}$. The vertices of $K$ correspond to clusters $V_{i_{1}}, \ldots, V_{i_{2 C+1}}$ in $G_{2}$; let $G_{K}$ denote the subgraph of $G_{2}^{\prime}$ induced by the vertices in these clusters combined. Every tuple $\left(V_{i_{j}}, V_{i_{k}}\right)$ of such clusters forms an $\varepsilon$-regular pair of density at least $\delta$ in $G_{K}$. Moreover, Lemma 4.4 implies that for each such cluster $V_{i_{j}}$, there is a subset $V_{i_{j}}^{\prime} \subseteq V_{i_{j}}$ of size $\left(1-\varepsilon^{1 / 2}\right) m$ so that $\left(V_{i_{j}}^{\prime}, V_{i_{k}}^{\prime}\right)$ forms an $\left(4 \varepsilon^{1 / 2}, \delta / 2\right)$-superregular pair in $G_{K}$ (for each $\left.1 \leq j \neq k \leq 2 C+1\right)$. The blow-up lemma (Lemma 4.6) now implies that $G_{K}$ contains a $2 C$-path covering all but at most $(2 C+1) \varepsilon^{1 / 2} m$ vertices in $G_{K}$.

Overall, this implies that $G_{2}$ contains a collection $\mathcal{P}$ of at most $\ell /(2 C+1) \leq M$ vertex-disjoint $2 C$-paths that together cover all but at most

$$
\left((2 C+1) \varepsilon^{1 / 2} m \times \frac{\ell}{2 C+1}\right)+\left(\eta_{3} \ell \times m\right)+\left|V_{0}\right| \leq \varepsilon^{1 / 2} n+\eta_{3} n+\varepsilon n \stackrel{(5.1)}{\leq} 2 \eta_{3} n
$$

vertices in $G_{2}$.

We will now use vertices in $G_{1}$ to connect all of the $2 C$-paths in $\mathcal{P} \cup\left\{K_{2 C+1}^{S}, K_{2 C+1}^{E}\right\}$ to obtain an $r$-path in $G$ whose first $2 C+1$ vertices are the vertices of $K_{2 C+1}^{E}$ and whose last $2 C+1$ vertices are the vertices of $K_{2 C+1}^{S}$. Note that we will have to reorder some of the vertices in the $2 C$-paths in $\mathcal{P}$, which is one reason we 'drop' from $2 C$-paths to an $r$-cycle. Label the $2 C$-paths in $\mathcal{P} \cup\left\{K_{2 C+1}^{S}, K_{2 C+1}^{E}\right\}$ by $P_{1}, \ldots, P_{t}$, where $P_{1}:=K_{2 C+1}^{E}$ and $P_{t}:=K_{2 C+1}^{S}$. In particular, note $M^{\prime} / 4 C \leq t \leq M+2$.

For each $P_{i}$, let $S_{i}$ denote the copy of $K_{C}$ induced by the first $C$ vertices on $P_{i}$; let $E_{i}$ denote the copy of $K_{C}$ induced by the last $C$ vertices on $P_{i}$; and let $P_{i}^{\prime}$ denote the $2 C$-path obtained from $P_{i}$ by deleting all vertices from $S_{i}$ and $E_{i}$. (Note that $P_{i}^{\prime}$ is certainly non-empty.)

Claim 5.4. Let $W \subseteq V\left(G_{1}\right)$ be arbitrary so that $|W| \leq \varepsilon n^{\prime}$. Given any $1 \leq i \leq t-1$, there is an $r$-path $P$ in $G$ so that:

(i) $V(P) \cap V\left(G_{2}\right)=E_{i} \cup S_{i+1}$.

(ii) $\left|V(P) \cap V\left(G_{1}\right)\right|=r$.

(iii) The first $C$ vertices on $P$ are precisely the vertices from $E_{i}$.

(iv) The last $C$ vertices on $P$ are precisely the vertices from $S_{i+1}$.

(v) $P$ is disjoint from $W$.

Proof (of claim). Apply Lemma 3.2 with $G, V^{\prime}, n^{\prime}, \eta_{3}, \sqrt{\rho}, d, E_{i}, S_{i+1}, W, r$ playing the roles of $G, U, n^{\prime}, \eta, \rho, d, X, Y, W, r$ to obtain a copy $K$ of $K_{r}$ in $G_{1}=G\left[V^{\prime}\right]$ such that $V(K) \cap W=\emptyset$ (recall that $E_{i} \cup S_{i+1}$ is disjoint from $\left.V^{\prime}\right)$ and there exist $E_{i}^{\prime} \subseteq E_{i}$ and $S_{i+1}^{\prime} \subseteq S_{i+1}$ such that $\left|E_{i}^{\prime}\right|=\left|S_{i+1}^{\prime}\right|=r$ and $E_{i}^{\prime} \cup S_{i+1}^{\prime} \subseteq N_{G}(K)$.

Altogether, this implies that $G_{1}$ contains the desired $r$-path $P$. Indeed, we construct $P$ so that the first $C-r$ vertices on $P$ are those vertices in $E_{i} \backslash E_{i}^{\prime}$ (in an arbitrary order); the next $r$ vertices are the elements from $E_{i}^{\prime}$; after that, we take the vertices from $K$ and then from $S_{i+1}^{\prime}$; the final $C-r$ vertices on $P$ are from $S_{i+1} \backslash S_{i+1}^{\prime}$.

With Claim 5.4 to hand, it is now easy to complete the proof of the theorem. Suppose that for some $j<t-1$, we have defined vertex-disjoint $r$-paths $P_{1}^{*}, \ldots, P_{j}^{*}$ such that, for each $i \leq j, P=P_{i}^{*}$ satisfies (i)-(iv) in Claim 5.4. Then define $W$ to be all those vertices in an $r$-path $P_{1}^{*}, \ldots, P_{j}^{*}$ that lie in $G_{1}$. So $|W|=j r \leq(M+2) r \leq \varepsilon n^{\prime}$. Claim 5.4 then implies there is an $r$-path $P_{j+1}^{*}$ in $G$ that satisfies the conclusion of Claim 5.4 (where $j+1$ plays the role of $i$ and $P_{j+1}^{*}$ the role of $P$ ).

Thus, we obtain vertex-disjoint $r$-paths $P_{1}^{*}, \ldots, P_{t}^{*}$ such that, for each $i \leq t, P=P_{i}^{*}$ satisfies (i)-(iv) in Claim 5.4. Consider the concatenation

$$
P^{*}:=S_{1} P_{1}^{\prime} P_{1}^{*} P_{2}^{\prime} P_{2}^{*} \ldots P_{t-1}^{\prime} P_{t-1}^{*} P_{t}^{\prime} E_{t} .
$$

This induces an $r$-path in $G$ (with many additional edges). Further, note that by the definition of $P_{1}$ (and thus $S_{1}$ ), the first $C$ vertices on $P^{*}$ lie in $K_{2 C+1}^{E}$ and so are adjacent in $G$ to every vertex in $E$. 
Similarly, the last $C$ vertices on $P^{*}$ lie in $K_{2 C+1}^{S}$ and so are adjacent in $G$ to every vertex in $S$. Thus, if we concatenate $P^{*}$ together with $P_{a b s}$, we obtain an $r$-cycle $C^{*}$ in $G$ (with many additional edges).

Note that, by (5.6), $C^{*}$ covers every vertex in $G$ except for at most $2 \eta_{3} n$ vertices in $G_{2}$ and at most $n^{\prime}=\eta_{3} n$ vertices in $G_{1}$. Since $3 \eta_{3} n<\eta_{2} n$, we may use the absorbing property (Claim 5.3(ii)) of $P_{a b s}$ to obtain the $r$ th power of a Hamilton cycle in $G$, as required.

\section{Lemmas for $H$}

Our rough aim is to find 'compatible' partitions of the vertex sets of $G$ and $H$ that allow us to apply the embedding lemmas (Lemmas 4.5 and 4.6) to complete the embedding of $H$ into $G$. In this section, we state and prove the so-called lemmas for $H$, whose input is some information about the structure of $G$ and whose output is a suitable partition of $H$.

\subsection{Partitioning a graph of low bandwidth: the basic lemma for $H$}

At some stage of the proof, $G$ will return some 'ideal' part sizes $\left\{m_{i, j}:(i, j) \in[\ell] \times[2 r]\right\}$, where $\chi(H) \leq r$. We would then like to find a suitable partition of $H$, the parts of which are close to these ideal sizes (equivalently, a mapping $f$ from $V(H)$ into $[\ell] \times[2 r]$ whose pre-images have controlled size). This is the purpose of the next lemma. It guarantees that $f$ is a graph homomorphism into $Z_{\ell}^{2 r}$ and produces a small set $B$ such that $f$ restricted to $V(H) \backslash B$ is a graph homomorphism into a $K_{2 r}$-factor (this is $(\mathscr{B} 3)$ ). Further, $(\mathscr{B} 4)$ says that for the first few vertices of $H$ (with respect to the bandwidth ordering of $H$ ), we have control of their images.

Before stating and proving Lemma 6.1, we would like to compare it to Lemma 8 in [9], the lemma for $H$ in the bandwidth theorem. There, the assumptions on $H$ are the same (in fact, slightly weaker), and the graph $Z_{\ell}^{2 r}$ mentioned above is replaced by a given graph $R$ of large minimum degree that contains a spanning subgraph $S$ (very similar to $Z_{\ell}^{r}$ ), which in turn contains a $K_{r}$-factor. Most edges are (and must be) mapped to the $K_{r}$-factor, which is much sparser than the $K_{2 r}$-factor we have at our disposal. This means the proof of Lemma 8 in [9] is much harder to prove than our Lemma 6.1. Despite this, our lemma does not follow from the statement of Lemma 8 in [9], so we prove it here.

Lemma 6.1 (Basic lemma for $H$ ). Let $n, r, \ell, \Delta \geq 1$ be integers, and let $\beta>0$ be such that $0<1 / n \ll$ $1 / r, 1 / \ell, 1 / \Delta, \beta$. Let $H$ be a graph on $n$ vertices with $\Delta(H) \leq \Delta$, and assume that $H$ has a labelling $x_{1}, \ldots, x_{n}$ of bandwidth at most $\beta n$ and $\chi(H) \leq r$. Furthermore, suppose $\left\{m_{i, j}:(i, j) \in[\ell] \times[2 r]\right\}$ is such that $\sum_{(i, j) \in[\ell] \times[2 r]} m_{i, j}=n ; m_{i, j} \geq 10 \beta n$ for all $(i, j) \in[\ell] \times[2 r] ;$ and $\left|m_{i, j}-m_{i, j^{\prime}}\right| \leq 1$ whenever $i \in[\ell]$ and $j, j^{\prime} \in[2 r]$. Let $\chi: V(H) \rightarrow[r]$ be a proper colouring of $H$. Then there exist a mapping $f: V(H) \rightarrow[\ell] \times[2 r]$ and a set of special vertices $B \subseteq V(H)$ with the following properties:

$(\mathscr{B} 1) \quad B \cap\left\{x_{1}, \ldots, x_{\beta n}\right\}=\emptyset$ and $|B| \leq 2 \ell \beta n$.

$(\mathscr{B} 2)|| f^{-1}(i, j)\left|-m_{i, j}\right| \leq 10 \beta n$ for every $(i, j) \in[\ell] \times[2 r]$.

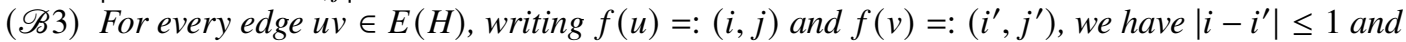
$j \neq j^{\prime}$. If, additionally, $u, v \notin B$, then $i=i^{\prime}$.

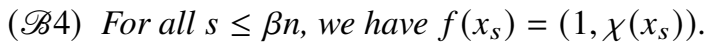

In particular, $f$ yields a homomorphism from $H$ to $Z_{\ell}^{2 r}$.

Note that the graph $Z_{\ell}^{2 r}$ that appears in Lemma 6.1 will be found in the reduced graph $R$ of $G$ : since $G$ is locally dense, $R$ is also locally dense (see Lemma 4.2); and thus, by Theorem 5.1, we can find a spanning $(4 r-1)$-cycle in $R$, which contains $Z_{\ell}^{2 r}$ (see (3.1)).

Recall that each vertex in $R$ corresponds to a unique cluster in $G$. In the proof of Theorem 1.2, the homomorphism $f$ from $H$ to $Z_{\ell}^{2 r} \subseteq R$ will be a guide as to which cluster in $G$ we should embed a vertex $x$ into for most vertices $x \in V(H)$. That is, roughly speaking, if $f(x)=(i, j) \in V(R)$, we embed $x$ into the cluster in $G$ corresponding to $(i, j)$. Note, though, that $f$ does not 'guide' us as to which vertices from 
$H$ we should embed into the exceptional set $V_{0}$ of $G$. So in the proof of Theorem 1.2, we in fact apply Lemma 6.1 to an almost spanning subgraph of $H$, rather than $H$ itself; the remaining part of $H$ is then embedded into $G$ via an additional lemma for $H$ (Lemma 6.2 in Section 6.2). In particular, Lemma 6.2 governs which vertices from $H$ are embedded into $V_{0}$. Property $(\mathscr{B} 4)$ of the homomorphism $f$ is used to ensure that we can 'fit' the two Lemmas for $H$ together to complete the embedding of $H$ into $G$.

The idea of the proof of Lemma 6.1 is to first obtain a proper $2 r$-colouring $\chi^{\prime}$ of $H$ such that in any initial segment $x_{1}, \ldots, x_{t}$ of the bandwidth ordering of $H$, every colour is used roughly the same number of times in $\chi^{\prime}$. This then allows us to define $f$ in a sequential way. That is, for some $t_{1}$, we map each $x_{j}$ in $\left\{x_{1}, \ldots, x_{t_{1}}\right\}$ to $\left(1, \chi^{\prime}\left(x_{j}\right)\right)$; then, for some $t_{2}$, we map each $x_{j}$ in $\left\{x_{t_{1}+1}, \ldots, x_{t_{2}}\right\}$ to $\left(2, \chi^{\prime}\left(x_{j}\right)\right)$, and so on.

Proof of Lemma 6.1. Let $N:=\lceil 1 /(2 \beta)\rceil$, and partition the ordered vertices $x_{1}, \ldots, x_{n}$ into consecutive intervals $A_{1}, A_{2}, \ldots, A_{2 N}$, each of length $\beta n$ (except possibly $A_{2 N}$, which could be smaller). We view each interval as being ordered with the inherited bandwidth ordering.

We will first define a (proper) $2 r$-colouring $\chi^{\prime}: V(H) \rightarrow[2 r]$ by iteratively defining colourings $\chi_{i}^{\prime}$ for $i \in[N]$ with the following properties:

$\mathscr{P}_{1}(i) \quad \chi_{i}^{\prime}: \bigcup_{2 \leq t \leq 2 i} A_{t} \rightarrow[2 r]$ is a proper colouring of $H\left[\bigcup_{2 \leq t \leq 2 i} A_{t}\right]$.

$\mathscr{P}_{2}(i)$ For all odd $2 \leq t \leq 2 i$, we have $\chi_{i}^{\prime}\left(A_{t}\right) \subseteq[r]$; and for all even $2 \leq t \leq 2 i$, we have $\chi_{i}^{\prime}\left(A_{t}\right) \subseteq[2 r] \backslash[r]$.

$\mathscr{P}_{3}(i)$ Writing $b_{i}^{j}(s):=\left|\left\{x \in \bigcup_{2 \leq t \leq 2 s} A_{t}: \chi_{i}^{\prime}(x)=j\right\}\right|$ for all $j \in[2 r]$ and $s \in[i]$, we have $\left|b_{i}^{j}(s)-b_{i}^{j^{\prime}}(s)\right| \leq \beta n$ for all $\left(j, j^{\prime}\right) \in[[2 r]]^{2}$ and $s \in[i]$.

For $\mathscr{P}_{3}(i)$, recall that $[[2 r]]^{2}:=[r]^{2} \cup([2 r] \backslash[r])^{2}$. Define $\chi_{1}^{\prime}: A_{2} \rightarrow[2 r]$ by setting $\chi_{1}^{\prime}(x)=\chi(x)+r$. Clearly this satisfies $\mathscr{P}_{1}(1)-\mathscr{P}_{3}(1)$, in particular as $\left|A_{2}\right| \leq \beta n$. Suppose we have defined $\chi_{i}^{\prime}$ for some $i<N$ satisfying $\mathscr{P}_{1}(i)-\mathscr{P}_{3}(i)$. By permuting the sets of colours $[r]$ and $[2 r] \backslash[r]$, we can obtain a new proper $2 r$-colouring $c_{1}$ of $H\left[\bigcup_{2 \leq t \leq 2 i} A_{t}\right]$ satisfying $\mathscr{P}_{1}(i)-\mathscr{P}_{3}(i)$ and with the additional property that

$$
\left|c_{1}^{-1}(1)\right| \geq \ldots \geq\left|c_{1}^{-1}(r)\right| \text { and }\left|c_{1}^{-1}(r+1)\right| \geq \ldots \geq\left|c_{1}^{-1}(2 r)\right| .
$$

Define $k: A_{2 i+1} \cup A_{2 i+2} \rightarrow[2 r]$ by setting

$$
k(x)= \begin{cases}\chi(x) & \text { if } x \in A_{2 i+1} \\ \chi(x)+r & \text { if } x \in A_{2 i+2} .\end{cases}
$$

Clearly, $k$ is a proper colouring of $H\left[A_{2 i+1} \cup A_{2 i+2}\right]$ since $\chi$ is. By permuting the sets of colours $[r]$ and $[2 r] \backslash[r]$, we can obtain a new proper colouring $c_{2}$ of $H\left[A_{2 i+1} \cup A_{2 i+2}\right]$ from $k$ such that

$$
\left|c_{2}^{-1}(1)\right| \leq \ldots \leq\left|c_{2}^{-1}(r)\right| \text { and }\left|c_{2}^{-1}(r+1)\right| \leq \ldots \leq\left|c_{2}^{-1}(2 r)\right|
$$

(note that the ordering is reversed compared to (6.1)). Finally, define $\chi_{i+1}^{\prime}$ by setting

$$
\chi_{i+1}^{\prime}(x)= \begin{cases}c_{1}(x) & \text { if } x \in \cup_{2 \leq t \leq 2 i} A_{t} \\ c_{2}(x) & \text { if } x \in A_{2 i+1} \cup A_{2 i}\end{cases}
$$

The fact that $\mathscr{P}_{2}(i+1)$ holds is clear from $\mathscr{P}_{2}(i)$ and the definitions of $c_{1}, k, c_{2}$, and $\chi_{i+1}^{\prime}$.

To see that $\mathscr{P}_{1}(i+1)$ holds, let $x, y \in \bigcup_{2 \leq t \leq 2 i+2} A_{t}$, where $x y \in E(H)$. We need to show that $\chi_{i+1}^{\prime}(x) \neq \chi_{i+1}^{\prime}(y)$. Let $2 \leq t, t^{\prime} \leq 2 i+2$ be such that $x \in A_{t}$ and $y \in A_{t^{\prime}}$. Then $\left|t-t^{\prime}\right| \leq 1$ since the intervals $A_{j}$ respect the bandwidth ordering and each one (except perhaps $A_{2 N}$ ) has size $\beta n$. If $\left|t-t^{\prime}\right|=1$, then $\mathscr{P}_{2}(i+1)$ implies that one of $\chi_{i+1}^{\prime}(x), \chi_{i+1}^{\prime}(y)$ lies in $[r]$ and the other in $[2 r] \backslash[r]$, as required. So we may assume that $t=t^{\prime}$. If $2 \leq t \leq 2 i$, then $\left(\chi_{i+1}^{\prime}(x), \chi_{i+1}^{\prime}(y)\right)=\left(c_{1}(x), c_{1}(y)\right)$. But $c_{1}$ is a proper colouring since it was obtained from $\chi_{i}^{\prime}$ by permuting colours, and $\chi_{i}^{\prime}$ is a proper colouring by $\mathscr{P}_{1}(i)$. 
Suppose that $t \in\{2 i+1,2 i+2\}$. Then, similarly, $\left(\chi_{i+1}^{\prime}(x), \chi_{i+1}^{\prime}(y)\right)=\left(c_{2}(x), c_{2}(y)\right)$, and $c_{2}$ is a proper colouring since it was obtained from the proper colouring $k$ by permuting colours. Thus $\mathscr{P}_{1}(i+1)$ holds.

For $\mathscr{P}_{3}(i+1)$, define for $j \in[2 r]$ and $s \in[i+1]$

$$
b_{i+1}^{j}(s):=\left|\left\{x \in \bigcup_{2 \leq t \leq 2 s} A_{t}: \chi_{i+1}^{\prime}(x)=j\right\}\right|
$$

and let $b_{i+1}^{j}:=b_{i+1}^{j}(i+1)=\left|\left(\chi_{i+1}^{\prime}\right)^{-1}(j)\right|$. Then (6.3) implies that $b_{i+1}^{j}=\left|c_{1}^{-1}(j)\right|+\left|c_{2}^{-1}(j)\right|$ for all $j \in[2 r]$. Now let $\left(j, j^{\prime}\right) \in[[2 r]]^{2}$. Clearly, $\left|b_{i+1}^{j}(s)-b_{i+1}^{j^{\prime}}(s)\right| \leq \beta n$ for all $s \in[i]$ since this is true for $\chi_{i}^{\prime}$ and hence $c_{1}$. So it remains to show that $\left|b_{i+1}^{j}-b_{i+1}^{j^{\prime}}\right| \leq \beta n$. Equations (6.1) and (6.2) imply that the quantities $\left|c_{1}^{-1}(j)\right|-\left|c_{1}^{-1}\left(j^{\prime}\right)\right|$ and $\left|c_{2}^{-1}(j)\right|-\left|c_{2}^{-1}\left(j^{\prime}\right)\right|$ are never both positive and never both negative, since $j$ and $j^{\prime}$ are in different orders. This implies that

$$
\begin{aligned}
\left|b_{i+1}^{j}-b_{i+1}^{j^{\prime}}\right| & =|| c_{1}^{-1}(j)|-| c_{1}^{-1}\left(j^{\prime}\right)|+| c_{2}^{-1}(j)|-| c_{2}^{-1}\left(j^{\prime}\right)|| \\
& \leq \max \left\{|| c_{1}^{-1}(j)|-| c_{1}^{-1}\left(j^{\prime}\right)||,|| c_{2}^{-1}(j)|-| c_{2}^{-1}\left(j^{\prime}\right)||\right\} .
\end{aligned}
$$

Note that ||$c_{2}^{-1}(j)|-| c_{2}^{-1}\left(j^{\prime}\right)|| \leq \beta n$. Further, $c_{1}$ was obtained from $\chi_{i}^{\prime}$ by permuting colours in $[r]$ and in $[2 r] \backslash[r]$, so there is some $\left(q, q^{\prime}\right) \in[[2 r]]^{2}$ for which $c_{1}^{-1}(j)=\left(\chi_{i}^{\prime}\right)^{-1}(q)$ and $c_{1}^{-1}\left(j^{\prime}\right)=\left(\chi_{i}^{\prime}\right)^{-1}\left(q^{\prime}\right)$. Thus ||$c_{1}^{-1}(j)|-| c_{1}^{-1}\left(j^{\prime}\right)||=\left|b_{i}^{q}(i)-b_{i}^{q^{\prime}}(i)\right|$, which is at most $\beta n$ by $\mathscr{P}_{3}(i)$. Thus $\mathscr{P}_{3}(i+1)$ holds.

Therefore, we can obtain a colouring $\chi_{N}^{\prime}: V(H) \backslash A_{1} \rightarrow[2 r]$ satisfying $\mathscr{P}_{1}(N)-\mathscr{P}_{3}(N)$. Finally, define $\chi^{\prime}: V(H) \rightarrow[2 r]$ by setting

$$
\chi^{\prime}(x)= \begin{cases}\chi_{N}^{\prime}(x) & \text { if } x \in V(H) \backslash A_{1} \\ \chi(x) & \text { if } x \in A_{1}\end{cases}
$$

The following properties hold:

(i) $\chi^{\prime}: V(H) \rightarrow[2 r]$ is a proper colouring.

(ii) For all odd $t \in[2 N]$, we have $\chi^{\prime}\left(A_{t}\right) \subseteq[r]$; and for all even $t \in[2 N]$, we have $\chi^{\prime}\left(A_{t}\right) \subseteq[2 r] \backslash[r]$.

(iii) Writing $d^{j}(s):=\left|\left\{x \in \bigcup_{t \in[s]} A_{t}: \chi^{\prime}(x)=j\right\}\right|$ for all $j \in[2 r]$ and $s \in[2 N]$, we have $\left|d^{j}(s)-d^{j^{\prime}}(s)\right| \leq 2 \beta n$ for all $\left(j, j^{\prime}\right) \in[[2 r]]^{2}$ and $s \in[2 N]$.

Let $M_{0}=n_{0}:=0$. For all $i \in[\ell]$, let $M_{i}:=\sum_{j \in[2 r]} m_{i, j}$; and $n_{i}:=\sum_{t \in[i]} M_{t}$. (Note that $n_{\ell}=n$.) Let $B_{i}:=\left\{x_{n_{i-1}+1}, \ldots, x_{n_{i}}\right\}$. So $B_{1}, \ldots, B_{\ell}$ is a partition of $V(H)$ that respects the bandwidth ordering, and each interval inherits the bandwidth ordering. Let

$$
B:=\bigcup_{2 \leq i \leq \ell}\left\{x_{n_{i-1}+1}, \ldots, x_{n_{i-1}+\beta n}\right\} \cup \bigcup_{1 \leq i \leq \ell-1}\left\{x_{n_{i}-\beta n+1}, \ldots, x_{n_{i}}\right\},
$$

and define $f: V(H) \rightarrow[\ell] \times[2 r]$ by setting

$$
f(x):=\left(i, \chi^{\prime}(x)\right) \text { if } x \in B_{i} .
$$

We claim that $f$ is the required mapping. Note $|B|=2(\ell-1) \beta n$, and if $t \leq n_{1}-\beta n$, then $x_{t} \notin B$. But

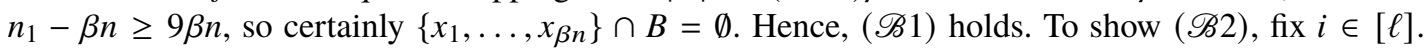
Choose the smallest $p^{-} \in[2 N]$ such that the first element of $A_{p-}$ lies in $B_{i}$ and the largest $p^{+} \in[2 N]$ such that the last element of $A_{p^{+}}$lies in $B_{i}$. So $B_{i}$ is the union of $\cup_{p^{-} \leq t \leq p^{+}} A_{t}$ together with a proper subset of $A_{p^{-}-1}$ and a proper subset of $A_{p^{+}+1}$. Thus,

$$
\left|f^{-1}(i, j)\right|=\left|\left\{x \in B_{i}: \chi^{\prime}(x)=j\right\}\right|=d^{j}\left(p^{+}\right)-d^{j}\left(p^{-}-1\right) \pm\left(\left|A_{p^{-}-1}\right|+\left|A_{p^{+}+1}\right|\right)
$$


for all $j \in[2 r]$. Let $\left(j, j^{\prime}\right) \in[[2 r]]^{2}$. Then the sizes of $f^{-1}(i, j)$ and $f^{-1}\left(i, j^{\prime}\right)$ do not differ much:

$$
\begin{aligned}
\left|f^{-1}(i, j)-f^{-1}\left(i, j^{\prime}\right)\right| & \stackrel{(6.6)}{\leq}\left|d^{j}\left(p^{+}\right)-d^{j^{\prime}}\left(p^{+}\right)\right|+\left|d^{j}\left(p^{-}-1\right)-d^{j^{\prime}}\left(p^{-}-1\right)\right|+4 \beta n \\
& \stackrel{(i i i))}{\leq} 8 \beta n .
\end{aligned}
$$

For any fixed $i \in[\ell]$,

$$
\begin{aligned}
& S_{1}:=\sum_{j \in[r]}\left|f^{-1}(i, j)\right| \stackrel{(i i),(6.5)}{=} \sum_{t \text { odd }}\left|B_{i} \cap A_{t}\right| \text { and } \\
& S_{2}:=\sum_{j \in[2 r] \backslash[r]}\left|f^{-1}(i, j)\right|=\sum_{t \text { even }}\left|B_{i} \cap A_{t}\right| .
\end{aligned}
$$

Therefore, $S_{1}+S_{2}=\left|B_{i}\right|=M_{i}$ and $\left|S_{1}-S_{2}\right| \leq \beta n$. So $S_{1}, S_{2}=M_{i} / 2 \pm \beta n$. By definition of $m_{i, j}$ and $M_{i}$, we have that $\left|m_{i, j}-M_{i} /(2 r)\right| \leq 1$ for all $j \in[2 r]$. Now let $j \in[r]$. We have

$$
\begin{aligned}
|| f^{-1}(i, j)\left|-m_{i, j}\right| & \leq|| f^{-1}(i, j)\left|-M_{i} /(2 r)\right|+1 \leq \frac{1}{r}|r| f^{-1}(i, j)\left|-S_{1}\right|+2 \beta n \\
& \leq \frac{1}{r} \sum_{j^{\prime} \in[r]}|| f^{-1}(i, j)|-| f^{-1}\left(i, j^{\prime}\right)||+2 \beta n \stackrel{(6.7)}{\leq} 10 \beta n,
\end{aligned}
$$

as required. The case when $j \in[2 r] \backslash[r]$ is almost identical. Thus ( $\mathscr{B} 2)$ holds.

Now let $u v \in E(H)$, and write $f(u)=:(i, j)$ and $f(v)=:\left(i^{\prime}, j^{\prime}\right)$ for $i, i^{\prime} \in[\ell]$ and $j, j^{\prime} \in[2 r]$. Since $\left|B_{t}\right|>\beta n$ for all $t \in[\ell]$ and $u \in B_{i}$ and $v \in B_{i^{\prime}}$, we have that $\left|i-i^{\prime}\right| \leq 1$ by consideration of the bandwidth ordering. We also have $j=\chi^{\prime}(u)$ and $j^{\prime}=\chi^{\prime}(v)$, and $\chi^{\prime}$ is a proper colouring of $H$, so $j \neq j^{\prime}$. Suppose additionally that $u, v \notin B$. If $i \neq i^{\prime}$, then $u$ and $v$ are separated by at least $2 \beta n$ in the bandwidth ordering, so $u v \notin E(H)$, a contradiction.

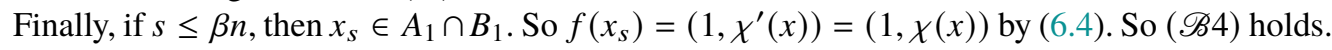

\subsection{Covering exceptional vertices: the second lemma for $H$}

The second lemma for $H$ will be used to find an embedding of a short initial segment of $H$ (in bandwidth ordering) into $G$ such that the exceptional set $V_{0}$, obtained after applying the regularity lemma, lies in the image of this embedding. In fact the pre-image of $V_{0}$ will be a 2 -independent set, which exists because $H$ has small maximum degree and bandwidth. As well as embedding this initial segment, we would like to find target sets for its neighbours so that eventually we can extend this embedding to the whole of $H$.

Lemma 6.2 (Special lemma for $H$ ). Let $n, r, L \geq 1$ be integers, and let $0<1 / n \ll \beta \ll 1 / L \ll \varepsilon \ll$ $\rho \ll \eta \ll d, 1 / r, 1 / \Delta$. Let $G$ be an $n$-vertex graph, $R$ be an L-vertex graph, and $\left\{b_{1}, \ldots, b_{r}\right\} \subseteq V(R)$ be such that

(G1) $G$ has vertex partition $\left\{V_{0}\right\} \cup\left\{V_{a}: a \in V(R)\right\}$ where $\left|V_{0}\right| \leq \varepsilon n$ and $\left|V_{a}\right|=:$ for all $a \in V(R)$.

(G2) Each $v \in V_{0}$ is equipped with a subset $N_{v} \subseteq V(R)$ with $\left|N_{v}\right| \geq \eta L$.

(G3) $R$ is $(\rho, d)$-dense, and $\delta(R) \geq(1 / 2+\eta) L$.

(G4) $R\left[\left\{b_{1}, \ldots, b_{r}\right\}\right] \cong K_{r}$, and $\left\{b_{1}, \ldots, b_{r}\right\}$ lies in a copy of $K_{18 r / \eta^{2}}$ in $R$.

Then there exists an integer $s \leq \varepsilon^{1 / 4} n$ such that the following holds. Let $H$ be a graph on $s+\beta n$ vertices with $\Delta(H) \leq \Delta$, and assume that $H$ has a labelling $x_{1}, \ldots, x_{s+\beta n}$ of bandwidth at most $\beta n$ and $\chi(H) \leq r$. Let $X:=\left\{x_{1}, \ldots, x_{s}\right\}$ and $Y:=\left\{x_{s+1}, \ldots, x_{s+\beta n}\right\}$. Let $\chi: V(H) \rightarrow[r]$ be a proper colouring of $H$. Then there exists a mapping $f: V(H) \rightarrow V(R) \cup V_{0}$ with the following properties:

(D1) Setting $I:=f^{-1}\left(V_{0}\right)$, we have that $I$ is a subset of $X$ that is 2-independent in $H$, and each vertex in $V_{0}$ is mapped onto from a unique vertex in $H$ (so $\left.|I|=\left|V_{0}\right|\right)$. 
(D2) For all $v \in V_{0}$, setting $W_{v}:=N_{H}\left(f^{-1}(v)\right)$, we have $W_{v} \subseteq X$ and $f\left(W_{v}\right) \subseteq N_{v}$.

(D3) $\left|f^{-1}(a)\right| \leq \varepsilon^{1 / 4} m$ for every $a \in V(R)$.

(D4) For every edge uv $\in E(H)$ such that $f(u), f(v) \notin V_{0}$, we have $f(u) f(v) \in E(R)$.

(D5) For all $y \in Y$, we have $f(y)=b_{\chi}(y)$.

To prove Lemma 6.2, we will need an auxiliary result, Lemma 6.3, which produces a 'framework' $F$ in the reduced graph that we will later use to find $f$. This framework $F$ is a $2 r$-trail such that for every $v \in V_{0}$, there is a copy $T$ of $K_{2 r}$ in $F$ such that $V(T) \subseteq N_{v}$.

Lemma 6.3. Let $0<1 / n \ll 1 / L \ll \varepsilon \ll \rho \ll \eta \ll d, 1 / r \leq 1$. Let $G$ be an $n$-vertex graph, $R$ be an $L$-vertex graph, and $\left\{b_{1}, \ldots, b_{r}\right\} \subseteq V(R)$ be such that

(G1) $G$ has vertex partition $\left\{V_{0}\right\} \cup\left\{V_{a}: a \in V(R)\right\}$ where $\left|V_{0}\right| \leq$ en and $\left|V_{a}\right|=:$ for all $a \in V(R)$.

(G2) Each $v \in V_{0}$ is equipped with a subset $N_{v} \subseteq V(R)$ with $\left|N_{v}\right| \geq \eta L$.

(G3) $R$ is $(\rho, d)$-dense, and $\delta(R) \geq(1 / 2+\eta) L$.

(G4) $R\left[\left\{b_{1}, \ldots, b_{r}\right\}\right] \cong K_{r}$, and $\left\{b_{1}, \ldots, b_{r}\right\}$ lies in a copy of $K_{18 r / \eta^{2}}$ in $R$.

Then there exist an integer $K \leq L^{2 r}$ and a subgraph $F \subseteq R$ such that

$(\mathscr{F} 1) F$ is a $2 r$-trail with ordering $a_{1}, \ldots, a_{t}$ where $t=(8 K+1) r$.

$(\mathscr{F} 2)$ There is a partition $V_{0}=V_{0}^{1} \cup \ldots \cup V_{0}^{K}$ such that $N_{v} \supseteq\left\{a_{8(i-1) r+1}, \ldots, a_{8(i-1) r+2 r}\right\}$ for all $v \in V_{0}^{i}$ and $\left|V_{0}^{i}\right| \leq \sqrt{\varepsilon} m / L^{2 r-1}$ for all $i \in[K]$.

(Fु) $\left(a_{t-r+1}, \ldots, a_{t}\right)=\left(b_{1}, \ldots, b_{r}\right)$.

$(\mathscr{F} 4)$ Every $a \in V(R)$ appears at most $L^{2 r-1} / \varepsilon^{1 / 12}$ times in the sequence $a_{1}, \ldots, a_{t}$.

Proof. Assume without loss of generality that $V(R)=[L]$. We first prove the following claim.

Claim 6.4. There is a $K \leq L^{2 r}$ and a set $\mathcal{T}=\left\{T_{1}, \ldots, T_{K}\right\}$ of $\left((d / 2)^{2 r} \eta L\right)$-extendable copies of $K_{2 r}$ in $R$ such that there is a partition $V_{0}=V_{0}^{1} \cup \ldots \cup V_{0}^{K}$ with the property that, for all $k \in[K]$, we have $\left|V_{0}^{k}\right| \leq \sqrt{\varepsilon} m / L^{2 r-1}$ and $T_{k} \subseteq R\left[N_{v}\right]$ for all $v \in V_{0}^{k}$.

Proof (of claim). By Lemma 3.1(i), we see that $R_{v}:=R\left[N_{v}\right]$ is $\left(\rho L^{2} /\left|N_{v}\right|^{2}, d\right)$-dense and hence $(\sqrt{\rho}, d)$-dense, where we used $(\mathscr{G} 2)$ and the fact that $\rho / \eta^{2}<\sqrt{\rho}$. Lemma 3.1(iv) implies that $R_{v}$ contains at least $\left.(d / 2){ }_{2}^{(2 r+1}\right) \eta^{2 r} L^{2 r} /(2 r)$ ! copies of $K_{2 r}$, each of which is $\left((d / 2)^{2 r} \eta L\right)$-extendable in $R_{v}$ (and thus $R$ ).

Let $T_{1}, \ldots, T_{K}$ be the set of $\left((d / 2)^{2 r} \eta L\right)$-extendable copies of $K_{2 r}$ in $R$. So

$$
K \leq\left(\begin{array}{c}
L \\
2 r
\end{array}\right) \leq L^{2 r}
$$

Then there is a partition $V_{0}^{1} \cup \ldots \cup V_{0}^{K}$ of $V_{0}$ into subsets (some of which may be empty) such that for all $k \in[K]$ and $v \in V_{0}^{k}$, we have that $R_{v} \supseteq T_{k}$ and

$$
\left|V_{0}^{k}\right| \leq \frac{\left|V_{0}\right|}{(d / 2)^{\left(\begin{array}{c}
2 r+1 \\
2
\end{array}\right)} \eta^{2 r} L^{2 r} /(2 r) !} \stackrel{(\mathscr{G} 1)}{\leq} \frac{\varepsilon m}{\left.(d / 2)^{(2 r+1}{ }_{2}\right) \eta^{2 r}(1-\varepsilon) L^{2 r-1} /(2 r) !} \leq \frac{\sqrt{\varepsilon} m}{L^{2 r-1}},
$$

as desired.

Let $\mathcal{T}:=\left\{T_{i}: i \in[K]\right\}$ be obtained from the claim. To complete the proof, we will use the connecting lemma (Lemma 3.3) to join the $K_{2 r} \mathrm{~s}$ in $\mathcal{T}$ into a $2 r$-trail. In so doing, we have to be careful not to visit any $a \in[L]$ too many times so as to ensure that $(\mathscr{F} 4)$ holds.

Suppose, for some $0 \leq i<K-1$ and all $j \in[i]$, we have obtained a copy $P_{j}=x_{j}^{1} \ldots x_{j}^{6 r}$ of $P_{6 r}^{2 r} \subseteq R$ such that

$\mathscr{P}_{1}(i) V\left(T_{j}\right) x_{j}^{1} \ldots x_{j}^{6 r}$ induces a copy $P_{j}^{\prime}$ of $P_{8 r}^{2 r}$. 
$\mathscr{P}_{2}(i) x_{j}^{1} \ldots x_{j}^{6 r} V\left(T_{j+1}\right)$ induces a copy $P_{j}^{\prime \prime}$ of $P_{8 r}^{2 r}$.

$\mathscr{P}_{3}(i)$ Each $a \in[L]$ lies in at most $\varepsilon^{-1 / 12} L^{2 r-1} / 2$ of the $2 r$-paths $P_{1}, \ldots, P_{i}$.

We would like to find $P_{i+1}$ such that $\mathscr{P}_{1}(i+1)-\mathscr{P}_{3}(i+1)$ hold. We will say that $a \in[L]$ is bad if it appears in at least $\varepsilon^{-1 / 12} L^{2 r-1} / 3$ of $P_{1}, \ldots, P_{i}$. Let $D$ be the set of bad $a$. Since each $P_{j}$ contains $6 r$ vertices, we have

$$
|D| \leq \frac{6 i r}{\varepsilon^{-1 / 12} L^{2 r-1} / 3} \leq \frac{18 \varepsilon^{1 / 12} K r}{L^{2 r-1}} \stackrel{(6.8)}{\leq} 18 \varepsilon^{1 / 12} r L .
$$

Recall from Claim 6.4 that $T_{i+1}$ and $T_{i+2}$ are both $\left((d / 2)^{2 r} \eta L\right)$-extendable copies of $K_{2 r}$ in $R$. Since $\eta \ll d, 1 / r$, they are $\eta^{2} L$-extendable copies. Apply Lemma 3.3 with $R, V\left(T_{i+1}\right), V\left(T_{i+2}\right), D, 2 r, \eta^{2}$ playing the roles of $G, X, Y, W, r, \eta$ to obtain a copy $P_{i+1}$ of $P_{6 r}^{2 r}=x_{i+1}^{1} \ldots x_{i+1}^{6 r}$ that avoids $D$ and such that $V\left(T_{i+1}\right) x_{i+1}^{1} \ldots x_{i+1}^{6 r}$ induces a copy $P_{i+1}^{\prime}$ of $P_{8 r}^{2 r}$, and $x_{i+1}^{1} \ldots x_{i+1}^{6 r} V\left(T_{i+2}\right)$ induces a copy $P_{i+1}^{\prime \prime}$ of $P_{8 r}^{2 r}$. So $\mathscr{P}_{1}(i+1)$ and $\mathscr{P}_{2}(i+1)$ hold. Now let $a \in[L]$. If $a \notin V\left(P_{i+1}\right)$, then $a$ lies in at most $\varepsilon^{-1 / 12} L^{2 r-1} / 2$ of $P_{1}, \ldots, P_{i+1}$ by $\mathscr{P}_{3}(i)$. Otherwise, since $P_{i+1}$ avoids $D, a$ lies in at most $\varepsilon^{-1 / 12} L^{2 r-1} / 3+1<$ $\varepsilon^{-1 / 12} L^{2 r-1} / 2$ of $P_{1}, \ldots, P_{i+1}$. So $\mathscr{P}_{3}(i+1)$ holds. Therefore, we can find $P_{1}, \ldots, P_{K-1}$ satisfying $\mathscr{P}_{1}(K-1)-\mathscr{P}_{3}(K-1)$.

Next we want to find a $2 r$-path between $T_{K}$ and $\left\{b_{1}, \ldots, b_{r}\right\}$. Let $\left\{b_{1}^{\prime}, \ldots, b_{r}^{\prime}\right\}$ be such that $\left\{b_{1}, \ldots, b_{r}, b_{1}^{\prime}, \ldots, b_{r}^{\prime}\right\}$ lies in a copy of $K_{18 r / \eta^{2}}$ in $R$ (such vertices exist by ( $\left.\mathscr{G} 4\right)$ ). Apply Lemma 3.3 with $R, V\left(T_{K}\right),\left\{b_{1}, \ldots, b_{r}, b_{1}^{\prime}, \ldots, b_{r}^{\prime}\right\}, \emptyset, 2 r, \eta^{2}$ playing the roles of $G, X, Y, W, r, \eta$ to obtain a copy $P_{K}$ of $P_{6 r}^{2 r}=x_{K}^{1} \ldots x_{K}^{6 r}$ such that $V\left(T_{K}\right) x_{K}^{1} \ldots x_{K}^{6 r}$ induces a copy $P_{K}^{\prime}$ of $P_{8 r}^{2 r}$, and furthermore $x_{K}^{1} \ldots x_{K}^{6 r} b_{1} \ldots b_{r} b_{1}^{\prime} \ldots b_{r}^{\prime}$ induces a copy of $P_{8 r}^{2 r}$; thus $x_{K}^{1} \ldots x_{K}^{6 r} b_{1} \ldots b_{r}$ induces a copy $P_{K}^{\prime \prime}$ of $P_{7 r}^{2 r}$. (Note that the vertices $b_{1}^{\prime}, \ldots, b_{r}^{\prime}$ were introduced only so that we could apply Lemma 3.3.) Clearly, $\mathscr{P}_{3}(K-1)$ implies that each $a \in[K]$ lies in at most $L^{2 r-1} \varepsilon^{-1 / 12} / 2+1$ of $P_{1}, \ldots, P_{K}$.

Writing $V\left(T_{i}\right)=\left\{y_{i}^{1}, \ldots, y_{i}^{2 r}\right\}$ for all $i \in[K], R$ contains a $2 r$-trail $F^{\prime}:=\bigcup_{i \in[K]}\left(P_{i}^{\prime} \cup P_{i}^{\prime \prime}\right)$ of length $(8 K+1) r=t$, with ordering given by

$$
\left(a_{1}, \ldots, a_{t}\right):=\left(y_{1}^{1}, \ldots y_{1}^{2 r}, x_{1}^{1}, \ldots x_{1}^{6 r}, y_{2}^{1}, \ldots y_{2}^{2 r}, \ldots, y_{K}^{1}, \ldots, y_{K}^{2 r}, x_{K}^{1}, \ldots, x_{K}^{6 r}, b_{1}, \ldots, b_{r}\right) .
$$

By construction, $(\mathscr{F} 1)$ and $(\mathscr{F} 3)$ hold.

We have that $V\left(T_{i}\right)=\left\{a_{8(i-1) r+1}, \ldots, a_{8(i-1) r+2 r}\right\}$ for all $i \in[K]$, which together with Claim 6.4 implies that $(\mathscr{F} 2)$ holds. Now let $a \in[L]$. Then $\mathscr{P}_{3}(K-1)$ implies that $a$ plays the role of some $x_{i}^{j}$ with $(i, j) \in[K] \times[6 r]$ at most $\varepsilon^{-1 / 12} L^{2 r-1} / 2+1$ times. Since each $T_{i}$ with $i \in[K]$ is a distinct copy of $K_{2 r}$ in $R$, we see that $a$ plays the role of some $y_{i}^{j}$ with $(i, j) \in[K] \times[2 r]$ at most $\left(\begin{array}{c}L-1 \\ 2 r-1\end{array}\right) \leq L^{2 r-1}$ times. Clearly, $a$ plays the role of at most one of $b_{1}, \ldots, b_{r}$. Thus the number of times $a$ appears in the sequence $a_{1}, \ldots, a_{t}$ is at most $\varepsilon^{-1 / 12} L^{2 r-1} / 2+L^{2 r-1}+2 \leq \varepsilon^{-1 / 12} L^{2 r-1}$. So (F्F 4$)$ holds.

Armed with Lemma 6.3, we can now prove Lemma 6.2. The proof proceeds by splitting $V(H)$ into segments and assigning each one to a copy of $K_{r}$ in $R$, according to the framework $F$. For example, the first segment of $V(H)$ will be assigned to $\left\{a_{1}, \ldots, a_{r}\right\}$; and, more specifically, those vertices coloured $i$ by $\chi$ will be mapped to $a_{i}$. In those special segments assigned to vertex sets of $K_{r} \mathrm{~s}$ that lie in $N_{v}$ for $v \in V_{0}^{i}$, we choose $\left|V_{0}^{i}\right|$ special vertices to be the pre-images of vertices in $V_{0}^{i}$. The property (F्F 4 ) of $F$ will ensure that not too many vertices are mapped to the same cluster of $R$.

Proof of Lemma 6.2. Let $G$ and $R$ be as in the statement of the lemma. Without loss of generality, we will assume that $V(R)=[L]$. Apply Lemma 6.3 to obtain $K \leq L^{2 r}$ and $F \subseteq R$ such that

$(\mathscr{F} 1) \quad F$ is a $2 r$-trail with ordering $a_{1}, \ldots, a_{t}$ where $t=(8 K+1) r$.

$(\mathscr{F} 2)$ There is a partition $V_{0}=V_{0}^{1} \cup \ldots \cup V_{0}^{K}$ such that $N_{v} \supseteq\left\{a_{8(i-1) r+1}, \ldots, a_{8(i-1) r+2 r}\right\}$ for all $v \in V_{0}^{i}$ and $\left|V_{0}^{i}\right| \leq \sqrt{\varepsilon} m / L^{2 r-1}$ for all $i \in[K]$.

(Fु) $\left(a_{t-r+1}, \ldots, a_{t}\right)=\left(b_{1}, \ldots, b_{r}\right)$. 
$(\mathscr{F} 4)$ Every $a \in[L]$ appears at most $L^{2 r-1} / \varepsilon^{1 / 12}$ times in the sequence $a_{1}, \ldots, a_{t}$.

Let

$$
s:=8 K \varepsilon^{1 / 3} m / L^{2 r-1} \leq 8 L \varepsilon^{1 / 3} m \stackrel{(\mathscr{S} 1)}{\leq} 8 \varepsilon^{1 / 3} n \leq \varepsilon^{1 / 4} n
$$

For all $i \in[K]$, let

$$
u_{i}:=\left|V_{0}^{i}\right| \stackrel{(\mathscr{F} 2)}{\leq} \sqrt{\varepsilon} m / L^{2 r-1} \text { and } \quad b:=\varepsilon^{1 / 3} m / L^{2 r-1}>100 \beta m L \stackrel{(\mathscr{G} 1)}{>} 99 \beta n .
$$

Let $H, X, Y$ be as in the statement of the lemma. Define a partition of $X \cup Y=\left\{x_{1}, \ldots, x_{s+\beta n}\right\}$ into $8 K+1$ intervals

$$
B_{1}^{1}, B_{1}^{2}, \ldots, B_{1}^{8}, B_{2}^{1}, B_{2}^{2}, \ldots, B_{2}^{8}, B_{3}^{1}, \ldots, B_{K}^{1}, B_{K}^{2}, \ldots, B_{K}^{8}, B_{K+1}^{1}
$$

where $\left|B_{i}^{j}\right|=b$ for all $(i, j) \in[K] \times[8] ;\left|B_{K+1}^{1}\right|=\beta n$; and the first $b$ vertices $x_{1}, \ldots, x_{b}$ in $X \cup Y$ form $B_{1}^{1}$, the next $b$ vertices in $X \cup Y$ form $B_{1}^{2}$, and so on. In particular, $B_{K+1}^{1}=Y$, and each interval comes equipped with the ordering inherited from the bandwidth ordering of $H$. The first claim identifies a set $I \subseteq X$ that will be the pre-image of $V_{0}$ in our desired mapping. Recall that given a graph $J$ and $A \subseteq V(J)$, we say that $A$ is 2-independent if every pair of vertices in $A$ is at distance at least 3 in $J$. In other words, $A$ is an independent set and, additionally, the neighbourhoods of different vertices in $A$ are disjoint.

Claim 6.5. For each $i \in[K]$, there exists a 2-independent set $I_{i} \subseteq B_{i}^{1}$ (with respect to $H$ ) of size $u_{i}$ such that $W(i):=\bigcup_{y \in I_{i}} N_{H}(y) \subseteq B_{i}^{1}$. Further, $I:=\bigcup_{i \in[K]} I_{i}$ is a 2-independent set in $H$.

Proof (of claim). Obtain $A_{i}$ from $B_{i}^{1}$ by removing the first $2 \beta n$ and last $2 \beta n$ elements (which is possible by (6.9)). Suppose we have obtained a 2-independent set $I^{j} \subseteq A_{i}$ of size $0 \leq j<u_{i}$. Then for any $y \in A_{i}$, the set $I^{j} \cup\{y\}$ is a 2-independent set in $H$ of size $j+1$ if $y \notin I^{j} \cup N_{H}\left(y^{\prime}\right) \cup N_{H}\left(N_{H}\left(y^{\prime}\right)\right)$ for any $y^{\prime} \in I^{j}$. The number of excluded $y$ is at most

$$
\begin{aligned}
\left|I^{j}\right|+\sum_{x \in I^{j}} d_{H}(x)+\sum_{x \in I^{j}} \sum_{z \in N_{H}(x)} d_{H}(z) & \leq\left|I^{j}\right|\left(1+\Delta+\Delta^{2}\right) \leq 2 \Delta^{2} u_{i} \\
& \stackrel{(6.9)}{\leq} 2 \Delta^{2} \sqrt{\varepsilon} m / L^{2 r-1} \stackrel{(6.9)}{<} b-4 \beta n=\left|A_{i}\right| .
\end{aligned}
$$

Therefore, we can find a 2-independent set $I_{i}:=I^{u_{i}}$ of size $u_{i}$ in $A_{i}$. This, together with the bandwidth property and the definition of $A_{i}$, implies that $W(i) \subseteq \bigcup_{y \in I_{i}}\left(N_{H}(y) \cup N_{H}\left(N_{H}(y)\right)\right) \subseteq B_{i}^{1}$. Thus there is no edge between $N_{H}\left(I_{i}\right)$ and $N_{H}\left(I_{i^{\prime}}\right)$ for $i \neq i^{\prime}$. So $I=\bigcup_{i \in[K]} I_{i}$ is a 2-independent set in $H$, proving the claim.

Let $\chi: V(H) \rightarrow[r]$ be the given proper colouring of $H$. A second claim finds a suitable homomorphism $\phi: V(H) \rightarrow V(F)$ on which $f$ will be based.

Claim 6.6. For each $(i, j) \in[K] \times[8] \cup\{(K+1,1)\}$, let

$$
\phi(x):=a_{(8(i-1)+(j-1)) r+\chi(x)} \quad \text { if } x \in B_{i}^{j} .
$$

Then $\phi: V(H) \rightarrow V(F)$ is a graph homomorphism such that $\left|\phi^{-1}(a)\right| \leq \varepsilon^{1 / 4} m$ for all $a \in[L]$.

Proof (of claim). Note first that if $a_{k}$ is in the image of $\phi$ for some $k \in \mathbb{N}$, then, recalling (F्F $)$, we have that $k \in[t]$, so $V(F) \supseteq \phi(V(H))$. Let us check that $\phi$ is a homomorphism. Let $x y \in E(H)$. Let $(i, j),\left(i^{\prime}, j^{\prime}\right) \in[K] \times[8] \cup\{(K+1,1)\}$ be such that $x \in B_{i}^{j}$ and $y \in B_{i^{\prime}}^{j^{\prime}}$. Since $H$ has bandwidth 
at most $\beta n$ and $\left|B_{i}^{j}\right|,\left|B_{i^{\prime}}^{j^{\prime}}\right|>\beta n$, we must have $\left(i^{\prime}, j^{\prime}\right) \in\{(i, j-1),(i, j),(i, j+1)\}$, where we let $(i, 9):=(i+1,1)$ and $(i, 0):=(i-1,8)$. So, writing

$$
T_{i}:=\left\{a_{(8(i-1)+(j-1)) r+p}: p \in[r]\right\} \quad \text { and } \quad T_{i^{\prime}}:=\left\{a_{\left(8\left(i^{\prime}-1\right) r+\left(j^{\prime}-1\right)\right)+p}: p \in[r]\right\}
$$

we either have $T_{i}=T_{i^{\prime}}$, or $T_{i}$ and $T_{i^{\prime}}$ are consecutive intervals in $a_{1}, \ldots, a_{t}$, each of length $r$. In both cases, we have $\phi(x) \neq \phi(y)$ (in the first case, this follows from the fact that $\chi(x) \neq \chi(y)$ ). But $(\mathscr{F} 1)$ now implies that $F\left[T_{i} \cup T_{i^{\prime}}\right]$ is a clique, so since $\phi(x) \in T_{i}$ and $\phi(y) \in T_{i^{\prime}}$ are distinct, $\phi(x) \phi(y) \in E(F)$, as required.

For the final assertion, each $a \in V(F)$ appears at most $L^{2 r-1} / \varepsilon^{1 / 12}$ times in the sequence $a_{1}, \ldots, a_{t}$ by $(\mathscr{F} 4)$. So, writing $\theta: V(H) \rightarrow[t]$ where $\phi(x)=a_{\theta(x)}$, we have

$$
\left|\phi^{-1}(a)\right| \leq \frac{L^{2 r-1}}{\varepsilon^{1 / 12}} \cdot \max _{k \in[t]}\left|\theta^{-1}(k)\right| \leq \frac{L^{2 r-1} b}{\varepsilon^{1 / 12}} \stackrel{(6.9)}{=} \varepsilon^{1 / 4} m,
$$

as desired.

Now let $H^{\prime}:=H \backslash I$, where $I:=\bigcup_{k \in[K]} I_{k}$ and $W:=\bigcup_{k \in[K]} W(k)$, where $W(k)$ is defined in Claim 6.5. Note also that $W \subseteq V\left(H^{\prime}\right)$ since, by Claim 6.5, $I$ is an independent set.

Let $g: I \rightarrow V_{0}$ be a bijection such that $g\left(I_{i}\right)=V_{0}^{i}$ for all $i \in[K]$ (which is clearly possible by Claim 6.5 and (6.9)). Since $I_{i}$ is a 2 -independent set in $H$, the set of neighbourhoods $N_{H}(y)$ is pairwise disjoint over all $y \in I_{i}$. So for each $w \in W(i)$, there is a unique $y \in I_{i}$ for which $w \in N_{H}(y)$. Claim 6.6 implies that $\left|\phi^{-1}(a)\right| \leq \varepsilon^{1 / 4} m$ for all $a \in[L]$.

We claim that $f: V(H) \rightarrow[L] \cup V_{0}$ given by

$$
f(x)= \begin{cases}\phi(x) & \text { if } x \in V(H) \backslash I \\ g(x) & \text { if } x \in I\end{cases}
$$

is the required mapping. Note that $f(V(H) \backslash I) \subseteq[L]$ and $f(I) \subseteq V_{0}$. For $(\mathscr{D} 1)$, note that, by Claim 6.5 and (6.10), $f(I)=g(I)=V_{0},|I|=\left|V_{0}\right|$ and $I$ is a 2-independent subset of $X$. For (D2), let $v \in V_{0}$ and $W_{v}:=N_{H}\left(f^{-1}(v)\right)$. Let $k \in[K]$ be such that $v \in V_{0}^{k}$. Then $f^{-1}(v)=g^{-1}(v) \in I_{k}$. So $W_{v} \subseteq W(i) \subseteq$ $B_{i}^{1} \subseteq X$. Let $x \in W_{v} \subseteq B_{i}^{1}$. By Claim 6.6 and $(\mathscr{F} 2)$, we have that $f(x)=\phi(x)=a_{8(i-1)+\chi(x)} \in N_{v}$. This completes the proof of $(\mathscr{D} 2)$.

For $(\mathscr{D} 3)$, let $a \in V(R)$. Then $f^{-1}(a) \subseteq \phi^{-1}(a)$ has size at most $\varepsilon^{1 / 4} m$ by Claim 6.6. For (D4), let $u v \in E(H)$ be such that $f(u), f(v) \notin V_{0}$. So $u, v \in V(H) \backslash I$ and $f(u)=\phi(u)$ and $f(v)=\phi(v)$. By Claim 6.6, $\phi: V(H) \rightarrow V(F)$ is a homomorphism, so $f(u) f(v) \in E(F) \subseteq E(R)$. Finally, for (D5), we have that $Y \cap I=\emptyset$ by Claim 6.5 , so for any $y \in Y$, we have $f(y)=\phi(y)=a_{t-r+\chi(y)}=b_{\chi}(y)$ by $(\mathscr{F} 3)$.

\section{The lemma for $G$ : adjusting cluster sizes}

Recall the definition of $Z_{\ell}^{r}$ from Section 3.1.1 and in particular that it contains a $K_{r}$-factor. Our goal in this section is to prove Lemma 7.1. Roughly speaking, it supposes that the reduced graph $R$ of $G$ contains a spanning copy of $Z_{\ell}^{2 r}$, its clusters $V_{1}, \ldots, V_{L}$ are equally sized, and pairs of clusters corresponding to the $K_{2 r}$-factor $\ell \cdot K_{2 r}$ in $Z_{\ell}^{2 r}$ are superregular. Then we can adjust $V_{1}, \ldots, V_{L}$ slightly by reallocating a small number of vertices so that they have given sizes, at the expense of now having superregular pairs corresponding to a $K_{r}$-factor $2 \ell \cdot K_{r}$.

To formalise the structural properties we need from $G$, we make the following definition (very similar to Definition 8.1 in [35]). 
Definition 1 ( $r$-Cycle structure). Given integers $n, \ell, r$, a graph $G$ on $n$ vertices, and constants $\varepsilon, \delta>0$, we say that $G$ has an $(R, \ell, r, \mathcal{V}, \varepsilon, \delta)$-cycle structure $\mathcal{C}$ if the following hold:



$(\mathscr{C} 2) R$ has vertex set $[\ell] \times[r]$ and $R \supseteq Z_{\ell}^{r}$ and $G\left[V_{i, j}, V_{i^{\prime}, j^{\prime}}\right]$ is $(\varepsilon, \delta)$-regular whenever $(i, j)\left(i^{\prime}, j^{\prime}\right) \in$ $E(R)$.

(C)3) $G\left[V_{i, j}, V_{i, j^{\prime}}\right]$ is $(\varepsilon, \delta)$-superregular whenever $i \in[\ell]$ and $1 \leq j<j^{\prime} \leq r$.

We say that $\mathcal{V}$ induces $\mathcal{C}$. If $V_{0}=\emptyset$, we say that $\mathcal{C}$ is spanning.

The next definition concerns a convenient relabelling of the vertex set of a graph, which we will use for the reduced graph $R$.

Definition 2 (Bijection $\phi_{\ell}^{2 r}$ ). Given integers $r, \ell$, define $\phi_{\ell}^{2 r}:[\ell] \times[2 r] \rightarrow[2 \ell] \times[r]$ by setting

$$
\phi_{\ell}^{2 r}(i, j)=\left((2 i-1)+\left\lfloor\frac{j}{r}\right\rfloor, j-\left(\left\lceil\frac{j}{r}\right\rceil-1\right) r\right), \quad \text { for all }(i, j) \in[\ell] \times[2 r] .
$$

It is easy to check that $\phi_{\ell}^{2 r}$ is a bijection and

$$
\begin{aligned}
& \phi_{\ell}^{2 r}(1,1) \ldots \phi_{\ell}^{2 r}(1, r) \phi_{\ell}^{2 r}(1, r+1) \ldots \phi_{\ell}^{2 r}(1,2 r) \ldots \phi_{\ell}^{2 r}(\ell, r+1) \ldots \phi_{\ell}^{2 r}(\ell, 2 r) \\
& =(1,1) \ldots \quad(1, r) \quad(2,1) \ldots \quad(2, r) \ldots \quad(2 \ell, 1) \ldots \quad(2 \ell, r) .
\end{aligned}
$$

This implies that for all $a \in[2 \ell]$ and distinct $b, b^{\prime} \in[r]$, there are $i \in[\ell]$ and $\left(j, j^{\prime}\right) \in[[2 r]]^{2}$ such that $\left(\phi_{\ell}^{2 r}(i, j), \phi_{\ell}^{2 r}\left(i, j^{\prime}\right)\right)=\left((a, b),\left(a, b^{\prime}\right)\right)$.

Given a graph $R$ and a bijection $\phi: V(R) \rightarrow V$ to some set $V$, we write $\phi(R)$ for the graph with vertex set $\{\phi(x): x \in V(R)\}$ and edge set $\{\phi(x) \phi(y): x y \in E(R)\}$. So $\phi(R) \cong R$.

In the language of Definition 1, the main result of this section states that, given a graph with a (spanning) $2 r$-cycle structure, we can obtain from it an $r$-cycle structure that is almost balanced, but the exact deviation from perfect balancedness can be controlled.

Lemma 7.1 (Lemma for $G$ ). Let $n, \ell, m, r \in \mathbb{N}$ and $0<1 / n \ll \xi \ll 1 / \ell \ll \varepsilon \ll \delta<1 / r$. Suppose that $G$ is a graph on $n$ vertices with a spanning $(R, \ell, 2 r, \mathcal{V}, \varepsilon, \delta)$-cycle structure, where $\mathcal{V}=\left\{V_{i, j}:(i, j) \in\right.$ $[\ell] \times[2 r]\}$ and $\left|V_{i, j}\right|=m$ for all $(i, j) \in[\ell] \times[2 r]$. Let $\left\{\tau_{a, b} \in \mathbb{Z}:(a, b) \in[2 \ell] \times[r]\right\}$ be such that $0 \leq \tau_{a, b} \leq \varepsilon m$ for all $(a, b) \in[2 \ell] \times[r]$. Then there exist positive integers $\left\{m_{a, b}:(a, b) \in[2 \ell] \times[r]\right\}$ such that

$(\mathscr{L} 1) \sum_{(a, b) \in[2 \ell] \times[r]}\left(m_{a, b}+\tau_{a, b}\right)=n$ and $m_{a, b} \geq(1-\sqrt{\varepsilon}) m$ and $\left|m_{a, b}-m_{a, b^{\prime}}\right| \leq 1$ for all $a \in[2 \ell]$ and $b, b^{\prime} \in[r]$.

(L2) Given any $\left\{n_{a, b} \in \mathbb{N}:(a, b) \in[2 \ell] \times[r]\right\}$ with $\sum_{(a, b) \in[2 \ell] \times[r]}\left(n_{a, b}+\tau_{a, b}\right)=n$ and $\mid m_{a, b}-$ $n_{a, b} \mid \leq \xi n$, there is a partition $\mathcal{X}=\left\{X_{a, b}:(a, b) \in[2 \ell] \times[r]\right\}$ of $V(G)$ with $\left|X_{a, b}\right|=n_{a, b}+\tau_{a, b}$ and $\left|X_{a, b} \Delta V_{\left(\phi_{\ell}^{2 r}\right)^{-1}(a, b)}\right| \leq \sqrt{\varepsilon} m$ for all $(a, b) \in[2 \ell] \times[r]$ such that $G$ has a spanning $\left(\phi_{\ell}^{2 r}(R), 2 \ell, r, X, \varepsilon^{1 / 3}, \delta / 2\right)$-cycle structure.

Proof. Note that

$$
2 r \ell m=n .
$$

For each $(i, j) \in[\ell] \times[2 r]$, choose $A_{i, j} \subseteq V_{i, j}$ satisfying

$$
\left|A_{i, j}\right|=\tau_{\phi_{\ell}^{2 r}(i, j)}
$$

and let

$$
Y_{i, j}:=V_{i, j} \backslash A_{i, j}, \quad \text { so } \quad(1-\varepsilon) m \leq\left|Y_{i, j}\right| \leq m .
$$


Let $y:=\left\{Y_{0}\right\} \cup\left\{Y_{i, j}:(i, j) \in[\ell] \times[2 r]\right\}$, where

$$
Y_{0}:=V(G) \backslash \bigcup_{(i, j) \in[\ell] \times[2 r]} Y_{i, j}=\bigcup_{(i, j) \in[\ell] \times[2 r]} A_{i, j} .
$$

Given a vertex $v \in V(G)$ and $(i, j) \in[\ell] \times[2 r]$, we will say that $v \rightarrow Y_{i, j}$ is valid if

$\circ j \in[r]$ and $d_{G}\left(v, Y_{i, j^{\prime}}\right) \geq(\delta-2 \varepsilon) m$ for all $j^{\prime} \in[r] \backslash\{j\}$; or

$\circ j \in[2 r] \backslash[r]$ and $d_{G}\left(v, Y_{i, j^{\prime}}\right) \geq(\delta-2 \varepsilon) m$ for all $j^{\prime} \in([2 r] \backslash[r]) \backslash\{j\}$.

The first claim furnishes us with many pairs $\left(v, Y_{i^{\prime}, j^{\prime}}\right)$ such that $v \in Y_{i, j}$ and $v \rightarrow Y_{i^{\prime}, j^{\prime}}$ is valid.

Claim 7.2. Let $i \in[\ell]$, and suppose that $1 \leq j \leq r<t \leq 2 r$ or $1 \leq t \leq r<j \leq 2 r$. Then every vertex $v \in Y_{i, j}$ is such that $v \rightarrow Y_{i, j}, Y_{i, t}$ is valid, and at least $(1-\sqrt{\varepsilon}) m$ are such that $v \rightarrow Y_{i+1, j}, Y_{i+1, t}$ are also valid. (Here, for example, $Y_{\ell+1, j}:=Y_{1, j}$.)

Proof (of claim). Let $t, j$ be as in the statement. Since, by $(\mathscr{C} 3), G\left[V_{i, j}, V_{i, j^{\prime}}\right]$ is $(\varepsilon, \delta)$-superregular for all $j^{\prime} \in[2 r] \backslash\{j\}$, we have that every vertex $v \in V_{i, j}$ has at least $\delta\left|V_{i, j^{\prime}}\right|$ neighbours in $V_{i, j^{\prime}}$. Thus every vertex $v \in Y_{i, j} \subseteq V_{i, j}$ has at least $\delta m-\varepsilon m \geq(\delta-2 \varepsilon) m$ neighbours in $Y_{i, j^{\prime}}$. In particular, $v \rightarrow V_{i, j}, V_{i, t}$ is valid.

From the definition of regularity, one can see the following. If $G[A, B]$ is an $(\varepsilon, \delta)$-regular graph, then there are fewer than $\varepsilon|A|$ vertices with fewer than $(\delta-\varepsilon)|B|$ neighbours in $B$. Thus, if $S_{i, j}$ is a subset of $N_{i, j}:=\left\{\left(i^{\prime}, j^{\prime}\right) \in V(R): G\left[V_{i, j}, V_{i^{\prime}, j^{\prime}}\right]\right.$ is $(\varepsilon, \delta)$-regular $\}$, we see that there are at least $\left(1-\varepsilon\left|S_{i, j}\right|\right)\left|V_{i, j}\right|$ vertices in $V_{i, j}$ with at least $(\delta-\varepsilon) m$ neighbours in $V_{i^{\prime}, j^{\prime}}$ for all $\left(i^{\prime}, j^{\prime}\right) \in S_{i, j}$, and hence at least $(\delta-2 \varepsilon) m$ neighbours in $Y_{i^{\prime}, j^{\prime}}$.

Recall that, since $Z_{\ell}^{2 r} \subseteq R$ by $(\mathscr{C} 2)$, we have that $N_{i, j} \supseteq\left\{\left(i, j^{\prime}\right),\left(i+1, j^{\prime}\right): j^{\prime} \in[2 r] \backslash\{j\}\right\}$. Thus the second assertion of the claim follows by taking $S_{i, j}:=\left\{\left(i+1, j^{\prime}\right): j^{\prime} \in[2 r] \backslash\{j, t\}\right\}$ and using the fact that $\left(1-\left|S_{i, j}\right| \varepsilon\right)\left|V_{i, j}\right|-\left|A_{i, j}\right| \geq(1-(2 r-2) \varepsilon) m-\varepsilon m \geq(1-\sqrt{\varepsilon}) m$.

Next we prove the following claim, which will give us a 'balanced' partition.

Claim 7.3. $V(G)$ has a partition $\left\{Y_{0}\right\} \cup\left\{U_{i, j}:(i, j) \in[\ell] \times[2 r]\right\}$ such that the following hold for all $i \in[\ell]$ :

(U1) ||$U_{i, j}|-| U_{i, j^{\prime}}|| \leq 1$ for all $\left(j, j^{\prime}\right) \in[[2 r]]^{2}$.

(U2) $\left|Y_{i, j} \triangle U_{i, j}\right| \leq$ rem for all $j \in[2 r]$.

(U3) If $j \in[r]$, then $U_{i, j} \backslash Y_{i, j} \subseteq \bigcup_{k \in[2 r] \backslash[r]} Y_{i, k}$; and if $k \in[2 r] \backslash[r]$, then $U_{i, k} \subseteq Y_{i, k}$.

Proof (of claim). Fix an $i \in[\ell]$, and, to simplify notation, let $A_{j}:=Y_{i, j}, a_{j}:=\left|A_{j}\right|, B_{j}:=Y_{i, r+j}$, and $b_{j}:=\left|B_{j}\right|$ for all $j \in[r]$. Suppose, without loss of generality, that $a_{1} \geq \ldots \geq a_{r}$ and $b_{1} \geq \ldots \geq b_{r}$. Let

$$
S:=\max \left\{\sum_{j \in[r]}\left(a_{1}-a_{j}\right), \sum_{j \in[r]}\left(b_{j}-b_{r}\right)\right\} \stackrel{(7.4)}{\leq} r \varepsilon m .
$$

Now let $A_{j}(0):=A_{j}$ and $B_{j}(0):=B_{j}$, and $a_{j}(0):=\left|A_{j}(0)\right|$ and $b_{j}(0):=\left|B_{j}(0)\right|$ for all $j \in[r]$. Do the following for each $0 \leq s<S$. Fix $t^{-}, t^{+} \in[r]$ such that $a_{t^{-}}(s) \leq a_{j}(s)$ and $b_{t^{+}}(s) \geq b_{j}(s)$ for all $j \in[r]$. Choose $x \in B_{t^{+}} \cap B_{t^{+}}(s)$, and let

$$
\begin{aligned}
& A_{j}(s+1):= \begin{cases}A_{j}(s) \cup\{x\} & \text { if } j=t^{-} \\
A_{j}(s) & \text { if } j \in[r] \backslash\left\{t^{-}\right\} ;\end{cases} \\
& B_{j}(s+1):= \begin{cases}B_{j}(s) \backslash\{x\} & \text { if } j=t^{+} \\
B_{j}(s) & \text { if } j \in[r] \backslash\left\{t^{+}\right\} .\end{cases}
\end{aligned}
$$


Let $a_{j}(s+1):=\left|A_{j}(s+1)\right|$ and $b_{j}(s+1):=\left|B_{j}(s+1)\right|$ for all $j \in[r]$. The following properties are clear:

(i) For all $0 \leq s<S$ and $j \in[r]$, we have $A_{j}(s) \supseteq A_{j}$ and $A_{j}(s) \backslash A_{j} \subseteq \bigcup_{k \in[r]} B_{k}$, and $B_{j}(s) \subseteq B_{j}$. Furthermore, for all $j \in[r]$, we have $\sum_{j \in[r]}\left|A_{j}(s) \backslash A_{j}\right|=\sum_{k \in[r]}\left|B_{k} \backslash B_{k}(s)\right|=s$.

(ii) Letting $s_{1}:=\sum_{j \in[r]}\left(a_{1}-a_{j}\right)$, we have that $a_{1}\left(s_{1}\right)=\ldots=a_{r}\left(s_{1}\right)=a_{1}$; and for each $s>s_{1}$, we have $\left|a_{j}(s)-a_{j^{\prime}}(s)\right| \leq 1$.

(iii) Letting $s_{2}:=\sum_{j \in[r]}\left(b_{j}-b_{r}\right)$, we have that $b_{1}\left(s_{2}\right)=\ldots=b_{r}\left(s_{2}\right)=b_{r}$; and for each $s>s_{2}$, we have $\left|b_{j}(s)-b_{j^{\prime}}(s)\right| \leq 1$.

Now let $U_{i, j}:=A_{j}(S)$ if $j \in[r]$ and $U_{i, j}:=B_{j-r}(S)$ if $j \in[2 r] \backslash[r]$. For $(\mathcal{U} 1)$, the fact that $S=\max \left\{s_{1}, s_{2}\right\}$ together with (ii) and (iii) implies that $\left|a_{j}(S)-a_{j^{\prime}}(S)\right| \leq 1$ and $\left|b_{j}(S)-b_{j^{\prime}}(S)\right| \leq 1$ for all $j, j^{\prime} \in[r]$. So ( $\left.\mathcal{U} 1\right)$ holds. For (U2), we have by (i) that

$$
\begin{gathered}
\left|U_{i, j} \Delta Y_{i, j}\right|=\left|U_{i, j} \backslash Y_{i, j}\right|=\left|A_{j}(S) \backslash A_{j}\right| \leq S \stackrel{(7.5)}{\leq} r \varepsilon m \quad \text { if } j \in[r], \quad \text { and } \\
\left|U_{i, j} \triangle Y_{i, j}\right|=\left|Y_{i, j} \backslash U_{i, j}\right|=\left|B_{j-r} \backslash B_{j-r}(S)\right| \leq S \stackrel{(7.5)}{\leq} r \varepsilon m \quad \text { if } j \in[2 r] \backslash[r] .
\end{gathered}
$$

Finally, (U3) follows immediately from (i).

The next claim shows that we can modify $\left\{U_{i, j}\right\}$ further to obtain a new partition with clusters of given sizes (each of which does not differ much from $\left|U_{i, j}\right|$ ).

Claim 7.4. Let $\left\{Y_{0}\right\} \cup\left\{U_{i, j}:(i, j) \in[\ell] \times[2 r]\right\}$ be any partition of $V(G)$ satisfying $(\mathcal{U} 1)-(\mathcal{U} 3)$. Let $\left\{n_{i, j}^{\prime}:(i, j) \in[\ell] \times[2 r]\right\}$ be such that $\sum_{(i, j) \in[\ell] \times[2 r]} n_{i, j}^{\prime}=\sum_{(i, j) \in[\ell] \times[2 r]}\left|U_{i, j}\right|$ and ||$U_{i, j}\left|-n_{i, j}^{\prime}\right| \leq \xi n$ for all $(i, j) \in[\ell] \times[2 r]$. Then $V(G)$ has a partition $\left\{Y_{0}\right\} \cup\left\{W_{i, j}:(i, j) \in[\ell] \times[2 r]\right\}$ such that the following hold for all $(i, j) \in[\ell] \times[2 r]$ :

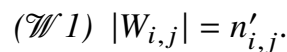

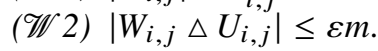

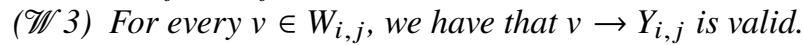

Proof (of claim). Let

$$
K:=2 r \ell \xi n \stackrel{(7.2)}{=} 4 r^{2} \ell^{2} \xi m \leq \frac{\varepsilon m}{2} .
$$

Suppose that, for some $0 \leq k<K / 2$, we have found for each $(i, j) \in[\ell] \times[2 r]$ subsets $U_{i, j}^{k} \subseteq V(G)$ such that the following hold:

$\mathscr{A}_{1}(k)\left\{Y_{0}\right\} \cup\left\{U_{i, j}^{k}:(i, j) \in[\ell] \times[2 r]\right\}$ is a partition of $V(G)$.

$\mathscr{A}_{2}(k)$ For all $v \in U_{i, j}^{k}$, we have that $v \rightarrow Y_{i, j}$ is valid.

$\mathscr{A}_{3}(k)$ For all $(i, j) \in[\ell] \times[2 r]$, we have $\left|U_{i, j}^{k} \Delta U_{i, j}\right| \leq 2 k$.

$\mathscr{A}_{4}(k) \sum_{(i, j) \in[\ell] \times[2 r]}|| U_{i, j}^{k}\left|-n_{i, j}^{\prime}\right| \leq 2(r \ell \xi n-k)$.

We claim that we can set $U_{i, j}^{0}:=U_{i, j}$ for all $(i, j) \in[\ell] \times[2 r]$. Indeed, $\mathscr{A}_{1}(0)$ holds by Claim 7.3. For $\mathscr{A}_{2}(0)$, let $(i, j) \in[\ell] \times[2 r]$, and let $v \in U_{i, j}$. If $v \in Y_{i, j}$, then $v \rightarrow Y_{i, j}$ is valid by Claim 7.2. Otherwise, $v \in U_{i, j} \backslash Y_{i, j}$. Note that by (U33), this implies $j \in[r]$ and, further, $v \in \bigcup_{k \in[2 r] \backslash[r]} Y_{i, k}$. So $v \rightarrow Y_{i, j}$ is valid by Claim 7.2. Property $\mathscr{A}_{3}(0)$ vacuously holds, and $\mathscr{A}_{4}(0)$ holds since ||$U_{i, j}\left|-n_{i, j}^{\prime}\right| \leq \xi n$ for all $(i, j) \in[\ell] \times[2 r]$.

If $\left|U_{i, j}^{k}\right|=n_{i, j}^{\prime}$ for all $(i, j) \in[\ell] \times[2 r]$, then we stop. Otherwise, we will obtain sets $U_{i, j}^{k+1}$ from $U_{i, j}^{k}$. There must exist $\left(i^{-}, j^{-}\right),\left(i^{+}, j^{+}\right) \in[\ell] \times[2 r]$ for which $\left|U_{i^{-}, j^{-}}^{k}\right| \leq n_{i^{-}, j^{-}}^{\prime}-1$ and $\left|U_{i^{+}, j^{+}}^{k}\right| \geq n_{i^{+}, j^{+}}^{\prime}+1$.

We will say that $\left(i_{1}, j_{1}\right) \rightarrow\left(i_{2}, j_{2}\right) \rightarrow \ldots \rightarrow\left(i_{s}, j_{s}\right)$ is a good chain (of length $s$ ) if for all $p \in[s-1]$, there exist at least $(1-\sqrt{\varepsilon}) m$ vertices $v \in Y_{i_{p}, j_{p}}$ such that $v \rightarrow Y_{i_{p+1}, j_{p+1}}$ is valid. Claim 7.2 implies that the following are good chains of length 3 (where here and for the remainder of the proof of Claim 7.4, 
addition is modulo $\ell$ ):

$$
\begin{aligned}
& \left(i^{+}, j^{+}\right) \rightarrow\left(i^{+}, j^{-}+r\right) \rightarrow\left(i^{+}+1, j^{-}\right) \quad \text { if } j^{+}, j^{-} \in[r] \\
& \left(i^{+}, j^{+}\right) \rightarrow\left(i^{+}, j^{-}-r\right) \rightarrow\left(i^{+}+1, j^{-}\right) \quad \text { if } j^{+}, j^{-} \in[2 r] \backslash[r] \\
& \left(i^{+}, j^{+}\right) \rightarrow\left(i^{+}, j^{-}\right) \rightarrow\left(i^{+}+1, j^{-}\right) \quad \text { otherwise, }
\end{aligned}
$$

and further, in all cases and for all $t \geq 0$, the chain $\left(i^{+}+t, j^{-}\right) \rightarrow\left(i^{+}+t+1, j^{-}\right)$of length 2 is good. Together, this implies that in all cases, there is a good chain

$$
\left(i^{+}, j^{+}\right)=:\left(i_{1}, j_{1}\right) \rightarrow \ldots \rightarrow\left(i_{S}, j_{S}\right):=\left(i^{-}, j^{-}\right)
$$

of some length $S$, where we choose the shortest such chain. As a crude estimate, we have, say, $S \leq 2 \ell$, and $\left(i_{s}, j_{s}\right) \neq\left(i_{s^{\prime}}, j_{s^{\prime}}\right)$ for any distinct $s, s^{\prime} \in[S]$ (or we could find a shorter chain).

We will exchange vertices between successive clusters according to this chain. For each $s \in[S]$, there are by definition at least $(1-\sqrt{\varepsilon}) m$ vertices $v \in Y_{i_{s}, j_{s}}$ such that $v \rightarrow Y_{i_{s+1}, j_{s+1}}$ is valid. The number of these vertices that additionally lie in $U_{i_{s}, j_{s}}^{k}$ is by $(\mathcal{U} 2), \mathscr{A}_{3}(0)$ and (7.6) at least $(1-\sqrt{\varepsilon}) m-2 k-r \varepsilon m>m / 2$. So we can find $x_{s} \in U_{i_{s}, j_{s}}^{k}$ such that $x_{s} \rightarrow Y_{i_{s+1}, j_{s+1}}$ is valid. For each $(i, j) \in[\ell] \times[2 r]$, set

$$
U_{i, j}^{k+1}= \begin{cases}U_{i, j}^{k} \backslash\left\{x_{1}\right\} & \text { if }(i, j)=\left(i_{1}, j_{1}\right) \\ U_{i, j}^{k} \cup\left\{x_{s-1}\right\} \backslash\left\{x_{s}\right\} & \text { if }(i, j)=\left(i_{s}, j_{s}\right) \text { for some } 2 \leq s<S \\ U_{i, j}^{k} \cup\left\{x_{S-1}\right\} & \text { if }(i, j)=\left(i_{S}, j_{S}\right) \\ U_{i, j}^{k} & \text { otherwise. }\end{cases}
$$

Property $\mathscr{A}_{1}(k+1)$ holds by $\mathscr{A}_{1}(k)$, the definition of $U_{i, j}^{k+1}$, and the fact that each pair in the chain is distinct. Property $\mathscr{A}_{2}(k)$ and the choice of $x_{s}$ imply that $\mathscr{A}_{2}(k+1)$ holds. We have

$$
\left|U_{i, j}^{k+1} \Delta Y_{i, j}\right| \leq\left|U_{i, j}^{k+1} \Delta U_{i, j}^{k}\right|+\left|U_{i, j}^{k} \Delta Y_{i, j}\right| \stackrel{\mathscr{A}_{3}(k)}{\leq} 2(k+1),
$$

proving $\mathscr{A}_{3}(k+1)$ (note here we are again using the fact that each pair in our chain is distinct). Finally, observe that ||$U_{i^{ \pm}, j^{ \pm}}^{k+1}\left|-n_{i^{ \pm}, j^{ \pm}}^{\prime}\right|=|| U_{i^{ \pm}, j^{ \pm}}^{k}\left|-n_{i^{ \pm}, j^{ \pm}}^{\prime}\right|-1$ and $\left|U_{i, j}^{k+1}\right|=\left|U_{i, j}^{k}\right|$ for all other pairs $(i, j)$. Therefore,

$$
\sum_{(i, j) \in[\ell] \times[2 r]}|| U_{i, j}^{k+1}\left|-n_{i, j}^{\prime}\right|=\sum_{(i, j) \in[\ell] \times[2 r]}|| U_{i, j}^{k}\left|-n_{i, j}^{\prime}\right|-2 \stackrel{\mathscr{A}_{4}(k)}{\leq} 2(r \ell \xi n-(k+1)),
$$

proving $\mathscr{A}_{4}(k+1)$. So, for each $0 \leq k \leq K / 2$, either the procedure has terminated or we are able to proceed to step $k+1$. Therefore, there is some $p \leq K / 2$ such that $\sum_{(i, j) \in[\ell] \times[2 r]}|| U_{i, j}^{p}\left|-n_{i, j}^{\prime}\right|=0$. Note that, by $\mathscr{A}_{3}(p)$, we have

$$
\left|U_{i, j}^{p} \Delta U_{i, j}\right| \leq 2 p \leq K \stackrel{(7.6)}{\leq} \varepsilon m
$$

for all $(i, j) \in[\ell] \times[2 r]$. Thus setting $W_{i, j}:=U_{i, j}^{p}$ yields the required partition.

Apply Claim 7.3 to obtain $\left\{U_{i, j}:(i, j) \in[\ell] \times[2 r]\right\}$ satisfying $(\mathscr{U} 1)-(\mathscr{U} 3)$.

Let $\phi:=\phi_{\ell}^{2 r}$ as in Definition 2. Let

$$
U_{a, b}^{\prime}:=U_{\phi^{-1}(a, b)} \quad \text { and } \quad m_{a, b}:=\left|U_{a, b}^{\prime}\right| \quad \text { for all }(a, b) \in[2 \ell] \times[r] .
$$


We claim that $\left\{m_{a, b}\right\}$ satisfies ( $\left.\mathscr{L} 1\right)$. Indeed, $(\mathscr{U} 1)$ implies that $\left|m_{a, b}-m_{a, b^{\prime}}\right| \leq 1$ for all $a \in[2 \ell]$ and $b, b^{\prime} \in[r]$, and further, writing $\phi^{-1}(a, b)=:(i, j)$,

$$
m_{a, b}=\left|U_{i, j}\right| \stackrel{(\mathscr{U} 2)}{\geq}\left|Y_{i, j}\right|-r \varepsilon m \stackrel{(7.3)}{\geq}\left|V_{i, j}\right|-(r+1) \varepsilon m=(1-(r+1) \varepsilon) m \geq(1-\sqrt{\varepsilon}) m .
$$

Finally,

$$
\sum_{(a, b) \in[2 \ell] \times[r]} m_{a, b}=\sum_{(i, j) \in[\ell] \times[2 r]}\left|U_{i, j}\right|=n-\left|Y_{0}\right|=n-\sum_{(a, b) \in[2 \ell] \times[r]} \tau_{a, b},
$$

so $(\mathscr{L} 1)$ holds.

Now let $\left\{n_{a, b} \in \mathbb{N}:(a, b) \in[2 \ell] \times[r]\right\}$ satisfy $\sum_{(a, b) \in[2 \ell] \times[r]}\left(n_{a, b}+\tau_{a, b}\right)=n$ and $\left|m_{a, b}-n_{a, b}\right| \leq$ $\xi n$. Let

$$
n_{i, j}^{\prime}:=n_{\phi(i, j)} \quad \text { for all }(i, j) \in[\ell] \times[2 r]
$$

Apply Claim 7.4 with input partition $\left\{Y_{0}\right\} \cup\left\{U_{i, j}:(i, j) \in[\ell] \times[2 r]\right\}$ and input sizes $\left\{n_{i, j}^{\prime}:(i, j) \in\right.$ $[\ell] \times[2 r]\}$ to obtain a partition $\left\{Y_{0}\right\} \cup\left\{W_{i, j}:(i, j) \in[\ell] \times[2 r]\right\}$ satisfying $(\mathscr{W} 1)-(\mathscr{W} 3)$. Let

$$
X_{a, b}:=W_{\phi^{-1}(a, b)} \cup A_{\phi^{-1}(a, b)} \quad \text { for all }(a, b) \in[2 \ell] \times[r] .
$$

We claim that $\mathcal{X}:=\left\{X_{a, b}:(a, b) \in[2 \ell] \times[r]\right\}$ is the required partition for $(\mathscr{L} 2)$. For all $(a, b) \in$ $[2 \ell] \times[r]$, we have

$$
\left|X_{a, b}\right| \stackrel{(7.8)}{=}\left|W_{\phi^{-1}(a, b)}\right|+\left|A_{\phi^{-1}(a, b)}\right| \stackrel{(7.3),(\mathscr{W} 1)}{=} n_{\phi^{-1}(a, b)}^{\prime}+\tau_{a, b} \stackrel{(7.7)}{=} n_{a, b}+\tau_{a, b},
$$

as required. Also, writing $(i, j):=\phi^{-1}(a, b) \in[\ell] \times[2 r]$ and recalling that $A_{i, j} \subseteq V_{i, j}$, we have

$$
\begin{gathered}
\left|X_{a, b} \Delta V_{\phi^{-1}(a, b)}\right| \stackrel{(7.8)}{\stackrel{(U 2),(\mathscr{W} 2)}{=}}\left|W_{i, j} \Delta V_{i, j}\right| \leq\left|W_{i, j} \Delta U_{i, j}\right|+\left|U_{i, j} \Delta V_{i, j}\right| \\
\stackrel{\leq}{\leq} 2 r \varepsilon m \leq 3 r \varepsilon\left|X_{a, b}\right| \leq \sqrt{\varepsilon} m .
\end{gathered}
$$

Lastly, we need to check that $X$ induces a $\left(\phi(R), 2 \ell, r, X, \varepsilon^{1 / 3}, \delta / 2\right)$-cycle structure. That is, we need to check that $(\mathscr{C} 1)-(\mathscr{C} 3)$ hold. Property $(\mathscr{W} 1)$ implies that $X=\left\{X_{a, b}:(a, b) \in[2 \ell] \times[r]\right\}=$ $\left\{W_{i, j} \cup A_{i, j}:(i, j) \in[\ell] \times[2 r]\right\}$ is a partition of $V(G)$. So $(\mathscr{C} 1)$ holds. Now, by (3.1) and Definition 2, we see that $\phi(R)$ has vertex set $[2 \ell] \times[r]$, and, since $Z_{\ell}^{2 r} \subseteq R$, we have $Z_{2 \ell}^{r} \subseteq \phi(R)$ (with the correct labelling). Let $(a, b),\left(a^{\prime}, b^{\prime}\right) \in E(\phi(R))$, and write $(i, j):=\phi^{-1}(a, b)$ and $\left(i^{\prime}, j^{\prime}\right)=\phi^{-1}\left(a^{\prime}, b^{\prime}\right)$. Then

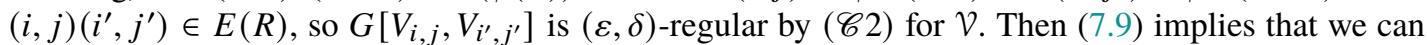
apply Proposition 4.3 with $\alpha:=3 r \varepsilon$ and $\varepsilon^{\prime}:=\varepsilon^{1 / 3} \geq \varepsilon+6 \sqrt{\alpha}$ to see that $G\left[X_{a, b}, X_{a^{\prime}, b^{\prime}}\right]$ is $\left(\varepsilon^{1 / 3}, \delta / 2\right)$ regular. So $(\mathscr{C} 2)$ holds.

For (C्C3), fix $a \in[2 \ell]$, and let $b, b^{\prime} \in[r]$ be distinct. Let $(i, j):=\phi^{-1}(a, b)$. Definition 2 implies that there exists $j^{\prime}$ such that $\left(j, j^{\prime}\right) \in[[2 r]]^{2}$ and $\phi^{-1}\left(a, b^{\prime}\right)=\left(i, j^{\prime}\right)$. Let $x \in X_{a, b} \backslash V_{\phi^{-1}(a, b)}=W_{i, j} \backslash Y_{i, j}$.

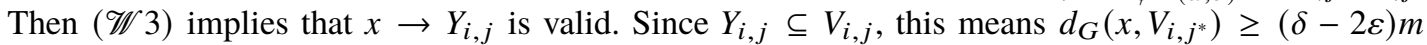
for all $j^{*}$ such that $\left(j, j^{*}\right) \in[[2 r]]^{2}$. So $d_{G}\left(x, V_{\phi^{-1}\left(a, b^{\prime}\right)}\right) \geq(\delta-2 \varepsilon) m$, and hence (7.9) implies $d_{G}\left(x, X_{a, b^{\prime}}\right) \geq(\delta-2 \varepsilon) m-2 r \varepsilon m \geq \delta\left|X_{a, b^{\prime}}\right| / 2$. Similarly, every $y \in X_{a, b^{\prime}} \backslash V_{\phi^{-1}\left(a, b^{\prime}\right)}$ satisfies $d_{G}\left(y, X_{a, b}\right) \geq \delta\left|X_{a, b}\right| / 2$. Moreover, (C्C3) for $\mathcal{V}$ and (7.9) implies that $d_{G}\left(x, X_{a, b^{\prime}}\right) \geq \delta\left|X_{a, b^{\prime}}\right| / 2$ for every $x \in V_{\phi^{-1}(a, b)}$ and $d_{G}\left(y, X_{a, b}\right) \geq \delta\left|X_{a, b}\right| / 2$ for every $y \in V_{\phi^{-1}\left(a, b^{\prime}\right)}$. So Proposition 4.3 applied


This completes the proof of $(\mathscr{L} 2)$ and hence of the lemma. 


\section{The proof of Theorem 1.2}

First, note that it suffices to prove the theorem under the additional assumption that $\eta \ll d, 1 / \Delta$. Let $n_{0}, \beta, \rho, \varepsilon, c, \delta, \rho^{\prime}, L^{\prime}>0$ satisfy

$$
0<1 / n_{0} \ll \beta \ll 1 / L^{\prime} \ll \rho \ll \varepsilon \ll c \ll \delta \ll \rho^{\prime} \ll \eta \ll d, 1 / \Delta .
$$

Let $G$ be a $(\rho, d)$-dense graph on $n \geq n_{0}$ vertices with $\delta(G) \geq(1 / 2+\eta) n$. Let $H$ be a graph on $n$ vertices with $\Delta(H) \leq \Delta$ and bandwidth at most $\beta n$. Write $r:=\chi(H)$; so as $\eta \ll 1 / \Delta$, certainly $\eta \ll 1 / r$.

Apply the regularity lemma (Lemma 4.1 ) with parameters $\varepsilon,(4 r+1) L^{\prime}$ to obtain $L^{*} \in \mathbb{N}$. We may assume that $\beta \ll 1 / L^{*}$.

Claim 8.1. There exists $L^{\prime} \leq \ell \leq L^{*}$, a partition $\mathcal{V}=\left\{V_{0}\right\} \cup\left\{V_{i, j}:(i, j) \in[\ell] \times[4 r]\right\}$ of $V(G)$ with $\left|V_{i, j}\right|=: m$ for all $(i, j) \in[\ell] \times[4 r]$, a graph $R$ on vertex set $[\ell] \times[4 r]$ and a spanning subgraph $G^{\prime}$ of $G$, such that

(i) $R$ is $\left(\rho^{\prime}, d\right)$-dense.

(ii) $\delta(R) \geq(1 / 2+\eta / 3)|R|$.

(iii) $G^{\prime}$ has an $\left(R, \ell, 4 r, \mathcal{V}, 7 \varepsilon^{1 / 4}, \delta / 2\right)$-cycle structure $\mathcal{C}$ and $\left|V_{0}\right| \leq 2 \varepsilon^{1 / 2} n$.

(iv) $R[\{(1,1), \ldots,(1,4 r)\}] \cong K_{4 r}$ and $\{(1,1), \ldots,(1,4 r)\}$ lies in a copy of $K_{324 r / \eta^{2}}$ in $R$.

Proof (of claim). Apply Lemma 4.1 to $G$ with parameters $\varepsilon, \delta,(4 r+1) L^{\prime}$ to obtain clusters $V_{1}, \ldots, V_{L}$ of size $m^{\prime}$, an exceptional set $V_{0}^{\prime}$, a pure graph $G^{\prime}$, and a reduced graph $R^{\prime}$. So

$$
L m^{\prime} \leq n \leq L m^{\prime}+\varepsilon n
$$

and $\left|R^{\prime}\right|=L$, where

$$
(4 r+1) L^{\prime} \leq L \leq L^{*}
$$

and $\left|V_{0}^{\prime}\right| \leq \varepsilon n$,

$$
\delta\left(G^{\prime}\right) \geq(1 / 2+\eta) n-(\delta+\varepsilon) n \geq(1 / 2+\eta / 2) n
$$

and $G^{\prime}\left[V_{i}, V_{j}\right]$ is $(\varepsilon, \delta)$-regular whenever $i j \in E\left(R^{\prime}\right)$. Lemma 4.2 implies that $R^{\prime}$ is $(3 \delta, d)$-dense and $\delta\left(R^{\prime}\right) \geq(1 / 2+\eta / 2) L$.

Let $r^{*}:=324 r / \eta^{2}$. Apply Theorem 5.1 with $R^{\prime}, L, r^{*}-1,4 r, 3 \delta, d, \eta / 2$ playing the roles of $G, n, r, s, \rho, d, \eta$ to obtain an $\left(r^{*}-1\right)$-cycle $C \cong C_{4 r \ell}^{r^{*}-1} \subseteq R^{\prime}$ of order $4 r \ell$ where

$$
(1-\varepsilon) L \leq L-4 r \leq 4 r \ell \leq L .
$$

Relabel those clusters of $R^{\prime}$ corresponding to vertices of $C$ so that they are now $\left\{V_{i, j}^{\prime}:(i, j) \in[\ell] \times[4 r]\right\}$, and

$$
(1,1)(1,2) \ldots(1,4 r)(2,1) \ldots(2,4 r) \ldots(\ell, 1) \ldots(\ell, 4 r)=C_{4 r \ell}^{r^{*}-1} \supseteq Z_{\ell}^{4 r} .
$$

Let $R:=R^{\prime}[V(C)]$. So $V(R)=[\ell] \times[4 r]$. Observe that $\{(1,1), \ldots,(1,4 r)\}$ lies in a copy of $K_{r^{*}}$ in $R$. For all $i \in[\ell]$, let

$$
T(i):=R\left[\bigcup_{j \in[4 r]}(i, j)\right] \stackrel{(8.6)}{\cong} K_{4 r}
$$

Apply Lemma 4.4 with $G^{\prime}\left[\bigcup_{j \in[4 r]} V_{i, j}^{\prime}\right], T(i), 4 r-1,4 r, V_{i, 1}^{\prime}, \ldots, V_{i, 4 r}^{\prime}, m^{\prime}, \varepsilon, \delta$ playing the roles of $G, R, \Delta, L, V_{1}, \ldots, V_{L}, m, \varepsilon, d$ to obtain for each $j \in[4 r]$ a subset $V_{i, j} \subseteq V_{i, j}^{\prime}$ of size

$$
\left|V_{i, j}\right|=m:=(1-\sqrt{\varepsilon}) m^{\prime}
$$


such that for every distinct $j, j^{\prime} \in[4 r]$, the graph $G^{\prime}\left[V_{i, j}, V_{i, j^{\prime}}\right]$ is $(4 \sqrt{\varepsilon}, \delta / 2)$-superregular. Let $V_{0}:=V(G) \backslash \bigcup_{(i, j) \in[\ell] \times[4 r]} V_{i, j}$ and

$$
\mathcal{V}:=\left\{V_{0}\right\} \cup\left\{V_{i, j}:(i, j) \in[\ell] \times[4 r]\right\} .
$$

We have

$$
\begin{aligned}
n & \geq 4 r \ell m \stackrel{(8.7)}{=} 4 r \ell(1-\sqrt{\varepsilon}) m^{\prime} \stackrel{(8.5)}{\geq}(1-\sqrt{\varepsilon})(1-\varepsilon) L m^{\prime} \stackrel{(8.2)}{\geq}(1-\sqrt{\varepsilon})(1-\varepsilon)^{2} n \\
& \geq\left(1-2 \varepsilon^{1 / 2}\right) n .
\end{aligned}
$$

Since we will often compare $m$ and $\beta n$ in calculations, let us note here that

$$
\beta n \stackrel{(8.2)}{\leq} \frac{\beta L m^{\prime}}{1-\varepsilon} \stackrel{(8.7)}{=} \frac{\beta L m}{(1-\sqrt{\varepsilon})(1-\varepsilon)} \stackrel{(8.1),(8.3)}{\leq} 2 \beta L^{*} \cdot m \leq \frac{\varepsilon^{2} m}{L^{*}} .
$$

We will now show that $\ell, R$, and $\mathcal{V}$ satisfy Claim 8.1(i)-(iv). We have that

$$
4 r L^{\prime} \stackrel{(8.1)}{\leq}(1-\varepsilon)(4 r+1) L^{\prime} \stackrel{(8.3)}{\leq}(1-\varepsilon) L \stackrel{(8.5)}{\leq} 4 r \ell \leq L \stackrel{(8.3)}{\leq} L^{*} .
$$

So $L^{\prime} \leq \ell \leq L^{*}$, as required. Note that (i) follows from Lemma 3.1(i) since $\rho^{\prime} \gg \delta$. Further, $\delta(R) \geq \delta\left(R^{\prime}\right)-4 r \geq(1 / 2+\eta / 3) L$, so (ii) holds.

For (iii), we need to show that $\mathcal{V}$ (see (8.8)) induces the required cycle structure $\mathcal{C}$. That is, we need to check that $(\mathscr{C} 1)-(\mathscr{C} 3)$ hold with the desired parameters. The sets $V_{i, j}$ are pairwise-disjoint since the same is true for $V_{i, j}^{\prime}$, so by the definition of $V_{0}$, we have that $\mathcal{V}$ is a partition of $V\left(G^{\prime}\right)$. Moreover,

$$
\left|V_{0}\right|=n-4 r \ell m \stackrel{(8.9)}{\leq} 2 \varepsilon^{1 / 2} n<7 \varepsilon^{1 / 4} n,
$$

so $(\mathscr{C} 1)$ holds. Certainly $V(R)=[\ell] \times[4 r]$ and, by (8.6), $R \supseteq Z_{\ell}^{4 r}$. Let $(i, j)\left(i^{\prime}, j^{\prime}\right) \in E(R)$. Then $(i, j)\left(i^{\prime}, j^{\prime}\right)$ has a corresponding edge in $R^{\prime} \supseteq R$, so $G^{\prime}\left[V_{i, j}^{\prime}, V_{i^{\prime}, j^{\prime}}^{\prime}\right]$ is $(\varepsilon, \delta)$-regular. Note that $\varepsilon+6 \sqrt{\varepsilon^{1 / 2}} \leq 7 \varepsilon^{1 / 4}$ and $\delta-4 \varepsilon^{1 / 2} \geq \delta / 2$. Thus Lemma 4.3 applied with $V_{i, j}^{\prime}, V_{i, j}, V_{i^{\prime}, j^{\prime}}^{\prime}, V_{i^{\prime}, j^{\prime}}, \varepsilon^{1 / 2}$ playing the roles of $A, A^{\prime}, B, B^{\prime}, \alpha$ implies that $G^{\prime}\left[V_{i, j}, V_{i^{\prime}, j^{\prime}}\right]$ is $\left(7 \varepsilon^{1 / 4}, \delta / 2\right)$-regular, so (C्C2) holds. We have already seen, for every $i \in[\ell]$ and distinct $j, j^{\prime} \in[4 r]$, that $G^{\prime}\left[V_{i, j}, V_{i, j^{\prime}}\right]$ is $(4 \sqrt{\varepsilon}, \delta / 2)$ superregular. Thus it is $\left(7 \varepsilon^{1 / 4}, \delta / 2\right)$-superregular. So $(\mathscr{C} 3)$ holds. Thus (iii) holds. We saw when we defined $R$ that (iv) holds. This completes the proof of the claim.

Recall the definition of the bijection $\phi_{\ell}^{4 r}:[\ell] \times[4 r] \rightarrow[2 \ell] \times[2 r]$ given by

$$
\phi_{\ell}^{4 r}(i, j)=\left((2 i-1)+\left\lfloor\frac{j}{2 r}\right\rfloor, j-\left(\left\lceil\frac{j}{2 r}\right\rceil-1\right) 2 r\right), \quad \text { for all }(i, j) \in[\ell] \times[4 r] .
$$

Recall also that $\phi_{\ell}^{4 r}(R)$ is the graph with vertex set $\phi_{\ell}^{4 r}(V(R))=[2 \ell] \times[2 r]$ and edge set $E\left(\phi_{\ell}^{4 r}(R)\right)=$ $\left\{\phi_{\ell}^{4 r}(x) \phi_{\ell}^{4 r}(y): x y \in E(R)\right\}$. For ease of notation, we will write

$$
\begin{aligned}
\phi & :=\phi_{\ell}^{4 r}, \quad \text { so } \quad \phi(1, b)=(1, b) \text { for all } b \in[2 r], \quad \text { and } \\
R^{*} & :=\phi(R), \quad \text { so } R^{*} \cong R, \quad V\left(R^{*}\right)=[2 \ell] \times[2 r],
\end{aligned}
$$

and $V\left(G^{\prime}\right)$ has partition $\mathcal{V}=\left\{V_{0}\right\} \cup\left\{V_{\phi^{-1}(a, b)}:(a, b) \in[2 \ell] \times[2 r]\right\}$.

Claim 8.2. There exists a partition $X=\left\{V_{0}\right\} \cup\left\{X_{a, b}:(a, b) \in[2 \ell] \times[2 r]\right\}$ of $V\left(G^{\prime}\right)$ and a surjective mapping $\psi: V(H) \rightarrow([2 \ell] \times[2 r]) \cup V_{0}$ such that the following hold: 
(i) $\left|\psi^{-1}(a, b)\right|=\left|X_{a, b}\right| \geq\left(1-\varepsilon^{1 / 19}\right) m$ for all $(a, b) \in[2 \ell] \times[2 r]$.

(ii) $G^{\prime}$ has an $\left(R^{*}, 2 \ell, 2 r, X, \varepsilon^{1 / 27}, \delta / 4\right)$-cycle structure $\mathrm{C}^{\prime}$.

(iii) $I:=\psi^{-1}\left(V_{0}\right)$ is an independent set in $H$ of size $\left|V_{0}\right|$; and for all $w \in W:=\bigcup_{x \in I} N_{H}(x)$, there is a unique $u \in I$ such that $u w \in E(H)$ and $d_{G^{\prime}}\left(\psi(u), X_{\psi(w)}\right) \geq \mathrm{cm} / 2$;

(iv) $\left.\psi\right|_{V(H) \backslash I}: V(H \backslash I) \rightarrow V\left(R^{*}\right)$ is a graph homomorphism.

(v) There exists $X^{\prime} \subseteq V(H) \backslash I$ with $W \subseteq X^{\prime}$ and $\left|\psi^{-1}(a, b) \cap X^{\prime}\right| \leq \varepsilon^{1 / 10} m$ for all $(a, b) \in[2 \ell] \times[2 r]$ such that, whenever $u v \in E(H)$ and $u, v \notin X^{\prime} \cup I$, writing $\psi(u)=:(a, b)$ and $\psi(v)=:\left(a^{\prime}, b^{\prime}\right)$, we have $a=a^{\prime}$ and $b \neq b^{\prime}$. Moreover, writing

$$
N:=\left(\bigcup_{x \in X^{\prime}} N_{H}(x)\right) \backslash\left(X^{\prime} \cup I\right)
$$

we have $|N| \leq \varepsilon m$.

Proof (of claim). For all $v \in V\left(G^{\prime}\right)$, write

$$
N_{R^{*}}^{c}(v):=\left\{(a, b) \in[2 \ell] \times[2 r]: d_{G^{\prime}}\left(v, V_{\phi^{-1}}(a, b)\right) \geq c m\right\}
$$

and $d_{R^{*}}^{c}(v):=\left|N_{R^{*}}^{c}(v)\right|$. Then

$$
(1 / 2+\eta) n-(\delta+\varepsilon) n \stackrel{(8.4)}{\leq} d_{G^{\prime}}(v) \leq d_{R^{*}}^{c}(v) m+\left(4 r \ell-d_{R^{*}}^{c}(v)\right) c m+\left|V_{0}\right| .
$$

Claim 8.1(iii) implies that

$$
4 r \ell m \leq n \leq 4 r \ell m+\left|V_{0}\right| \leq 4 r \ell m+2 \varepsilon^{1 / 2} n
$$

Thus

$$
d_{R^{*}}^{c}(v) \geq \frac{(1 / 2+\eta-\delta-\varepsilon) n-4 r \ell c m-\left|V_{0}\right|}{(1-c) m} \stackrel{(8.1)}{\geq} \frac{1 / 2+\eta / 2}{1-c} \cdot 4 r \ell \geq \frac{\left|R^{*}\right|}{2} .
$$

We would like to apply Lemma 6.2 (special lemma for $H$ ) to obtain an integer $s$, with $G^{\prime}, R^{*}, 4 r \ell$, $2 r, \eta / 3,2 \varepsilon^{1 / 2}, \rho^{\prime}, d, n, m, N_{R^{*}}^{c}(v),(1, i)$ playing the roles of $G, R, L, r, \eta, \varepsilon, \rho, d, n, m, N_{v}, b_{i}$. For this, we need to check that $(\mathscr{G} 1)-(\mathscr{G} 4)$ hold. For $(\mathscr{G} 1)$, we know that $G^{\prime}$ has vertex partition $\left\{V_{0}\right\} \cup\left\{V_{i, j}\right.$ : $(i, j) \in[\ell] \times[4 r]\}=\left\{V_{0}\right\} \cup\left\{V_{\phi^{-1}(a, b)}:(a, b) \in[2 \ell] \times[2 r]\right\}$, and $\left|V_{0}\right| \leq \varepsilon^{1 / 2} n$ and $\left|V_{p}\right|=m$ for all $p \in V\left(R^{*}\right)$. That ( $\mathscr{G}$ ) holds follows from (8.13). Property ( $\left.\mathscr{G} 3\right)$ follows from Claim 8.1(i) and (ii) and the fact that $R^{*} \cong R$. Finally, $(\mathscr{G} 4)$ follows from (iv), noting that $324 r / \eta^{2}=18 \cdot(2 r) \cdot 1 /(\eta / 3)^{2}$, and the fact that $\phi(1, b)=(1, b)$ for all $b \in[2 r]$ from (8.11). Therefore, we can apply Lemma 6.2 with the above parameters to obtain an integer

$$
s \leq\left(2 \varepsilon^{1 / 2}\right)^{1 / 4} n \leq \varepsilon^{1 / 9} n .
$$

Let $\chi: V(H) \rightarrow[r]$ be a proper colouring of $H$, let $x_{1}, \ldots, x_{n}$ be an ordering of $V(H)$ with bandwidth at most $\beta n$, and let

$$
\begin{aligned}
X & :=\left\{x_{1}, \ldots, x_{s}\right\}, \quad Y:=\left\{x_{s+1}, \ldots, x_{s+\beta n}\right\} \subseteq Z:=\left\{x_{s+1}, \ldots, x_{n}\right\} \\
H^{\prime} & :=H[X \cup Y] \text { and } H^{\prime \prime}:=H[Z] .
\end{aligned}
$$

Apply Lemma 6.2 (special lemma for $H$ ) with the above parameters and with $s, \beta, \Delta, H^{\prime}, X, Y, \chi$ playing the roles of $s, \beta, \Delta, H, X, Y, \chi$ to obtain a mapping

$$
f: X \cup Y \rightarrow([2 \ell] \times[2 r]) \cup V_{0}
$$

with the following properties: 
(D1) Setting $I:=f^{-1}\left(V_{0}\right)$, we have that $I$ is a subset of $X$ that is 2-independent in $H^{\prime}$, and each vertex in $V_{0}$ is mapped onto from a unique vertex in $H$ (so $|I|=\left|V_{0}\right|$ ).

(D2) For all $v \in V_{0}$, setting $W_{v}:=N_{H}\left(f^{-1}(v)\right)$, we have $W_{v} \subseteq X$ and $f\left(W_{v}\right) \subseteq N_{R^{*}}^{c}(v)$.

(D3) $\left|f^{-1}(a, b)\right| \leq \varepsilon^{1 / 9} m$ for every $(a, b) \in[2 \ell] \times[2 r]$.

(D4) For every edge $u v \in E(H)$ such that $f(u), f(v) \notin V_{0}$, we have $f(u) f(v) \in E\left(R^{*}\right)$.

(D5) For all $y \in Y$, we have $f(y)=(1, \chi(y))$.

Let

$$
\tau_{a, b}:=\left|\left(\left.f\right|_{X \backslash I}\right)^{-1}(a, b)\right|=\left|\left(\left.f\right|_{X}\right)^{-1}(a, b)\right| \text { for all }(a, b) \in[2 \ell] \times[2 r] .
$$

Then $0 \leq \tau_{a, b} \leq \varepsilon^{1 / 9} m$ for all $(a, b) \in[2 \ell] \times[2 r]$ by $(\mathscr{D} 3)$.

Apply Lemma 7.1 (the lemma for $G$ ) with $n-\left|V_{0}\right|, \ell, m, 2 r, 11 \beta, \varepsilon^{1 / 9}, \delta / 2, G^{\prime} \backslash V_{0}, R, \mathcal{V} \backslash\left\{V_{0}\right\}$ playing the roles of $n, \ell, m, r, \xi, \varepsilon, \delta, G, R, \mathcal{V}$ to obtain positive integers $\left\{m_{a, b}:(a, b) \in[2 \ell] \times[2 r]\right\}$ such that

$(\mathscr{L} 1) \sum_{(a, b) \in[2 \ell] \times[2 r]}\left(m_{a, b}+\tau_{a, b}\right)=n-\left|V_{0}\right|$ and $m_{a, b} \geq\left(1-\varepsilon^{1 / 18}\right) m$ and $\left|m_{a, b}-m_{a, b^{\prime}}\right| \leq 1$ for all $a \in[2 \ell]$ and $b, b^{\prime} \in[2 r]$.

$(\mathscr{L} 2)$ Given any $\left\{n_{a, b}:(a, b) \in[2 \ell] \times[2 r]\right\}$ with $\sum_{(a, b) \in[2 \ell] \times[2 r]}\left(n_{a, b}+\tau_{a, b}\right)=n-\left|V_{0}\right|$ and $\left|m_{a, b}-n_{a, b}\right| \leq 11 \beta\left(n-\left|V_{0}\right|\right)$, there is a partition $X=\left\{V_{0}\right\} \cup\left\{X_{a, b}:(a, b) \in[2 \ell] \times[2 r]\right\}$ of $V\left(G^{\prime}\right)$ with $\left|X_{a, b}\right|=n_{a, b}+\tau_{a, b}$ and $\left|X_{a, b} \Delta V_{\phi^{-1}(a, b)}\right| \leq \varepsilon^{1 / 18} m$ for all $(a, b) \in[2 \ell] \times[2 r]$ such that $G^{\prime}$ has an $\left(R^{*}, 2 \ell, 2 r, X, \varepsilon^{1 / 27}, \delta / 4\right)$-cycle structure.

Note that Lemma 7.1 yields a partition of $G^{\prime} \backslash V_{0}$ into clusters, and the partition of $V\left(G^{\prime}\right)$ specified in $(\mathscr{L} 2)$ is simply this partition together with $V_{0}$.

The next step is to apply Lemma 6.1 (basic lemma for $H$ ) to $H^{\prime \prime}=H[Z]$ (which overlaps with $H^{\prime}$ in $Y)$. Note that the number of vertices in $H^{\prime \prime}$ is $n-s \geq\left(1-\varepsilon^{1 / 9}\right) n$. Further,

$$
\sum_{(a, b) \in[2 \ell] \times[2 r]} m_{a, b} \stackrel{(\mathscr{L} 1)}{=} n-\left|V_{0}\right|-\sum_{(a, b) \in[2 \ell] \times[2 r]} \tau_{a, b} \stackrel{(8.17)}{=} n-\left|V_{0}\right|-|X \backslash I| \stackrel{(\mathscr{D} 1)}{=} n-|X| \stackrel{(8.15)}{=}|Z|
$$

and $m_{a, b} \geq\left(1-\varepsilon^{1 / 18}\right) m \geq 10 \beta(n-s)$ by (8.1). Thus we can apply Lemma 6.1 with $n-$ $s, r, 2 \ell, \Delta, \beta, H^{\prime \prime},\left(x_{s+1}, \ldots, x_{n}\right), \chi,\left\{m_{a, b}:(a, b) \in[2 \ell] \times[2 r]\right\}$ playing the roles of $n, r, \ell, \Delta, \beta, H$, $\left(x_{1}, \ldots, x_{n}\right), \chi,\left\{m_{a, b}:(a, b) \in[2 \ell] \times[2 r]\right\}$ to obtain a mapping

$$
k: Z \rightarrow[2 \ell] \times[2 r]
$$

and $B \subseteq Z$ with the following properties:

$(\mathscr{B} 1) \quad B \subseteq Z \backslash Y$ and $|B| \leq 2 \ell \beta n$.

$(\mathscr{B} 2)|| k^{-1}(a, b)\left|-m_{a, b}\right| \leq 10 \beta n$ for every $(a, b) \in[2 \ell] \times[2 r]$.

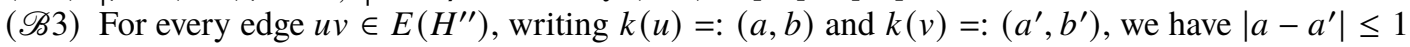
and $b \neq b^{\prime}$. If additionally $u, v \notin B$, then $a=a^{\prime}$.

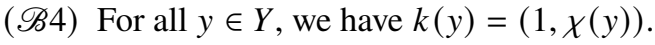

Let

$$
n_{a, b}:=\left|k^{-1}(a, b)\right| \text { for all }(a, b) \in[2 \ell] \times[2 r] .
$$

Then

$$
\sum_{(a, b) \in[2 \ell] \times[2 r]} n_{a, b} \stackrel{(8.19),(8.20)}{=}|Z| \stackrel{(8.18)}{=} \sum_{(a, b) \in[2 \ell] \times[2 r]} m_{a, b} \stackrel{(\mathscr{L} 2)}{=} n-\left|V_{0}\right|-\sum_{(a, b) \in[2 \ell] \times[2 r]} \tau_{a, b},
$$

and for all $(a, b) \in[2 \ell] \times[2 r]$,

$$
\left|n_{a, b}-m_{a, b}\right| \stackrel{(8.20)}{=}|| k^{-1}(a, b)\left|-m_{a, b}\right| \stackrel{(\mathscr{B} 2)}{\leq} 10 \beta n \leq 11 \beta\left(n-\left|V_{0}\right|\right) .
$$


Thus ( $\mathscr{L} 2)$ implies that there is a partition $X=\left\{V_{0}\right\} \cup\left\{X_{a, b}:(a, b) \in[2 \ell] \times[2 r]\right\}$ of $V\left(G^{\prime}\right)$ with, for all $(a, b) \in[2 \ell] \times[2 r]$,

$$
\left|X_{a, b}\right|=\left|k^{-1}(a, b)\right|+\left|\left(\left.f\right|_{X \backslash I}\right)^{-1}(a, b)\right| \quad \text { and } \quad\left|X_{a, b} \Delta V_{\phi^{-1}(a, b)}\right| \leq \varepsilon^{1 / 18} m
$$

such that $G^{\prime}$ has an $\left(R^{*}, 2 \ell, 2 r, X, \varepsilon^{1 / 27}, \delta / 4\right)$-cycle structure.

Define a mapping $\psi: V(H) \rightarrow([2 \ell] \times[2 r]) \cup V_{0}$ by setting

$$
\psi(x)= \begin{cases}f(x) & \text { if } x \in X \\ k(x) & \text { if } x \in Z\end{cases}
$$

Finally, let $X^{\prime}:=(X \backslash I) \cup B$.

We need to check that $X, \psi$, and $X^{\prime}$ satisfy Claim 8.2(i)-(v). For (i), we have

$$
\begin{aligned}
\left|\psi^{-1}(a, b)\right| & \stackrel{(8.22)}{=}\left|k^{-1}(a, b)\right|+\left|\left(\left.f\right|_{X \backslash I}\right)^{-1}(a, b)\right| \stackrel{(8.21)}{=}\left|X_{a, b}\right| \stackrel{(\mathscr{B} 2)}{\geq} m_{a, b}-10 \beta n \\
& \stackrel{(\mathscr{L} 1)}{\geq}\left(1-\varepsilon^{1 / 18}\right) m-10 \beta n \stackrel{(8.10)}{\geq}\left(1-\varepsilon^{1 / 19}\right) m .
\end{aligned}
$$

Further, we have already seen that (ii) holds.

Note that $I=f^{-1}\left(V_{0}\right)=\psi^{-1}\left(V_{0}\right)$ has size $\left|V_{0}\right|$ and is a 2-independent subset of $X$ in $H^{\prime}$ by ( $\left.\mathscr{D} 1\right)$. Let $w \in W:=\bigcup_{x \in I} N_{H}(x)$. Since $I$ is 2-independent, there is a unique $u \in I \subseteq X$ such that $u w \in E(H)$. So $w \in W_{f(u)}=W_{\psi(u)} \subseteq X$ in the notation of $(\mathscr{D} 2)$. So $\psi(w)=f(w) \in N_{R^{*}}^{c}(f(u))=N_{R^{*}}^{c}(\psi(u))$. Thus

$$
d_{G^{\prime}}\left(\psi(u), X_{\psi(w)}\right) \stackrel{(8.21)}{\geq} d_{G^{\prime}}\left(\psi(u), V_{\phi^{-1}(\psi(w))}\right)-\varepsilon^{1 / 18} m \stackrel{(8.12)}{\geq} \mathrm{cm} / 2,
$$

so (iii) holds.

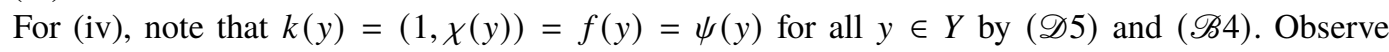
that $\psi^{\prime}:=\left.\psi\right|_{V(H) \backslash I}$ is a map into $V\left(R^{*}\right)=[2 \ell] \times[2 r]$. Let $x y \in E(H)$ where $x, y \notin I$. Suppose first that $x, y \in X \cup Y$. Then $\psi(x)=f(x)$ and $\psi(y)=f(y)$. Then $f(x), f(y) \notin V_{0}$, so (D4) implies that $f(x) f(y) \in E\left(R^{*}\right)$. Suppose now that $x, y \in Z$. Write $\psi(x)=k(x)=(a, b)$ and $\psi(y)=k(y)=\left(a^{\prime}, b^{\prime}\right)$, where $(a, b),\left(a^{\prime}, b^{\prime}\right) \in[2 \ell] \times[2 r]$. Then $(\mathscr{B} 3)$ implies that $\left|a-a^{\prime}\right| \leq 1$ and $b \neq b^{\prime}$. Thus $\psi(x) \psi(y) \in$ $E\left(Z_{2 \ell}^{2 r}\right) \subseteq E\left(R^{*}\right)$, as required. The only other possibility is that one of $x, y$ is in $X$ and the other is in $Z \backslash Y$. But then the distance between them in the bandwidth ordering of $H$ is more than $|Y|=\beta n$, a contradiction to $x y \in E(H)$. Thus $\psi^{\prime}: V(H \backslash I) \rightarrow V\left(R^{*}\right)$ is a graph homomorphism. So (iv) holds.

For (v), note that $B \subseteq Z$, so $X^{\prime} \cap I=\emptyset$; and $W=\bigcup_{v \in V_{0}} W_{v} \subseteq X$; and $W \cap I=\emptyset$ since $I$ is 2-independent in $H^{\prime}$. So $W \subseteq X^{\prime}$. Now let $(a, b) \in[2 \ell] \times[2 r]$. We have

$$
\begin{gathered}
\left|\psi^{-1}(a, b) \cap X^{\prime}\right| \leq\left|\psi^{-1}(a, b) \cap X\right|+|B| \leq\left|f^{-1}(a, b)\right|+|B| \\
\stackrel{(\mathscr{D} 3),(\mathscr{B} 1)}{\leq} \varepsilon^{1 / 9} m+2 \ell \beta n \stackrel{(8.10)}{\leq} \varepsilon^{1 / 10} m .
\end{gathered}
$$

Now let $u v \in E(H)$, where $u, v \notin X^{\prime} \cup I$. So $u, v \in Z \backslash B$. Write $\psi(u)=(a, b)$ and $\psi(v)=\left(a^{\prime}, b^{\prime}\right)$.

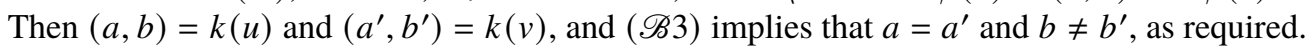

Finally, define $N$ as in (v). If $y \in N$, then either $y \in \bigcup_{x \in X} N_{H}(x) \backslash X \subseteq Y$ or $y \in \bigcup_{x \in B} N_{H}$ (x) (or both). So (8.15) and the fact that $\Delta(H) \leq \Delta$ imply that

$$
|N| \leq|Y|+\Delta|B| \leq(2 \Delta \ell+1) \beta n \stackrel{(8.1),(8.10)}{\leq} \varepsilon m .
$$

This completes the proof of (v) and hence of the claim.

In the final part of the proof, we will use the cycle structure $\mathcal{C}^{\prime}$, mapping $\psi$ and special set $X^{\prime}$ obtained in Claim 8.2 to find an embedding $g$ of $H$ into $G^{\prime} \subseteq G$. We will do this in three stages: (1) define an embedding $g_{1}$ of $I$ into $V_{0}$, according to $\psi ;(2)$ find an embedding $g_{2}$ of $X^{\prime}$ using $\psi$ as a framework, 
such that there are large candidate sets for the neighbouring vertices $N$ of $X^{\prime}$; (3) find an embedding $g_{3}$ of the remainder of $H$ using the blow-up lemma, using the candidate sets obtained in (2) to ensure that $g_{2}$ is compatible with $g_{3}$. Then set $g$ to be the union of $g_{1}, g_{2}, g_{3}$.

Stage (1) is easy; we simply define

$$
g_{1}: I \rightarrow V_{0} \quad \text { where } \quad g_{1}(x):=\psi(x) \text { for all } x \in I .
$$

Since by Claim 8.2(iii), $I$ is an independent set in $H$ of size $V_{0}$, we trivially have that $g_{1}$ is an embedding of $H[I]$ into $V\left(G^{\prime}\right)$.

For Stage (2), we will apply Lemma 4.5 (embedding lemma with target sets) to embed vertices in $X^{\prime}$. Indeed, let $\psi^{*}:=\left.\psi\right|_{X^{\prime} \cup N}$. Given $w \in W$, let $u$ be the unique element of $I$ such that $u w \in E(H)$, as guaranteed by Claim 8.2(iii). Let

$$
S_{w}:=N_{G^{\prime}}\left(\psi(u), X_{\psi(w)}\right) .
$$

We will apply Lemma 4.5 with $G^{\prime} \backslash V_{0}, R^{*}, H\left[X^{\prime} \cup N\right], n-\left|V_{0}\right|, 4 r \ell, \varepsilon^{1 / 27}, c / 2, \delta / 4, \Delta$, $\left\{X_{a, b}\right\},\left(1-\varepsilon^{19}\right) m, \psi^{*}, X^{\prime}, N, W, S_{w}$ playing the roles of $G, R, H, n, L, \varepsilon, c, \delta, \Delta,\left\{V_{a}: a \in\right.$ $V(R)\}, m, \phi, X, Y, W, S_{w}$. To see why this is possible, note that, by Claim 8.1(iii), $G^{\prime} \backslash V_{0}$ has

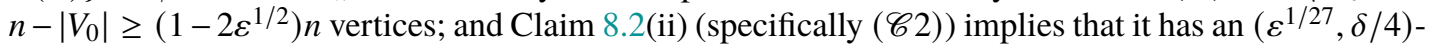
regular partition $\left\{X_{a, b}:(a, b) \in V\left(R^{*}\right)\right\}$. Clearly, as a restriction of $\psi$, the function $\psi^{*}$ is a suitable graph homomorphism, and by Claim 8.2(v) and (8.23), we have

$$
\left|\left(\psi^{*}\right)^{-1}(a, b)\right| \leq\left|\psi^{-1}(a, b) \cap X^{\prime}\right|+|N| \leq \varepsilon^{1 / 10} m+\varepsilon m \leq \varepsilon^{1 / 12} m .
$$

Finally, $W \subseteq X^{\prime}$ by Claim $8.2(\mathrm{v})$, and $\left|S_{w}\right| \geq \mathrm{cm} / 2$ by Claim 8.2(iii). So the above are suitable parameters for the application of Lemma 4.5.

Thus there is a mapping

$$
g_{2}: X^{\prime} \rightarrow V\left(G^{\prime}\right) \backslash V_{0}
$$

that is an embedding of $H\left[X^{\prime}\right]$ into $G^{\prime}$ such that

$(\mathscr{T} 1) g_{2}(x) \in X_{\psi^{*}(x)}$ for all $x \in X^{\prime}$.

$(\mathscr{T} 2) g_{2}(w) \in S_{w}$ for all $w \in W$.

(T3) For all $y \in N$, there exists $C_{y} \subseteq X_{\psi^{*}(y)} \backslash g_{2}\left(X^{\prime}\right)$ such that $C_{y} \subseteq N_{G^{\prime}}\left(g_{2}(x)\right)$ for all $x \in$ $N_{H}(y) \cap\left(X^{\prime}\right)$, and $\left|C_{y}\right| \geq c m / 2$.

For Stage (3), we will do the following for each $a \in[2 \ell]$. Let $U_{a, b}:=X_{a, b} \backslash g_{2}\left(X^{\prime}\right)$ for all $b \in[2 r]$. We want to show that $U_{a, b}$ has exactly the right size to embed the remaining vertices of $H$ whose image under $\psi$ is $(a, b)$. Indeed, let $\psi^{\prime}:=\left.\psi\right|_{H \backslash\left(X^{\prime} \cup I\right)}$. Then Claim 8.2(i) implies that

$$
\left|U_{a, b}\right|=\left|X_{a, b}\right|-\left|g_{2}\left(X^{\prime}\right) \cap X_{a, b}\right| \stackrel{(\mathscr{T} 1)}{=}\left|\psi^{-1}(a, b)\right|-\left|\left(\psi^{*}\right)^{-1}(a, b) \cap X^{\prime}\right|=\left|\left(\psi^{\prime}\right)^{-1}(a, b)\right|
$$

where we used the fact that $\psi^{-1}(a, b) \cap I=\emptyset$. This together with (8.25) implies that $\left|U_{a, b} \Delta X_{a, b}\right|=$ $\left|\left(\psi^{*}\right)^{-1}(a, b) \cap X^{\prime}\right| \leq \varepsilon^{1 / 10} m \leq 2 \varepsilon^{1 / 10}\left|U_{a, b}\right|$. Let $b, b^{\prime} \in[2 r]$ be distinct. So $\left|U_{a, b}\right| \geq\left(1-\varepsilon^{1 / 20}\right) m$ by Claim 8.2(i). Recall from Claim 8.2(ii) (specifically $(\mathscr{C} 3))$ that $G^{\prime}\left[X_{a, b}, X_{a, b^{\prime}}\right]$ is $\left(\varepsilon^{1 / 27}, \delta / 4\right)$ superregular. So given any $x \in U_{a, b}$, Claim 8.2(i) implies that

$$
d_{G^{\prime}}\left(x, U_{a, b^{\prime}}\right) \geq \delta\left|X_{a, b}\right| / 4-\varepsilon^{1 / 10} m \geq\left(\delta / 4-\delta \varepsilon^{1 / 19}-\varepsilon^{1 / 10}\right) m \geq \delta\left|U_{a, b}\right| / 5 .
$$

Thus Proposition 4.3 with $G^{\prime}, X_{a, b}, U_{a, b}, X_{a, b^{\prime}}, U_{a, b^{\prime}}, \varepsilon^{1 / 27}, \delta / 4, \varepsilon^{1 / 10}$ playing the roles of $G, A, A^{\prime}, B, B^{\prime}, \varepsilon, \delta, \alpha$ implies that $G^{\prime}\left[U_{a, b}, U_{a, b^{\prime}}\right]$ is $\left(2 \varepsilon^{1 / 27}, \delta / 5\right)$-superregular for all distinct $b, b^{\prime} \in$ [2r]. The set $N$ has size at most $\varepsilon m \leq 2 \varepsilon\left|U_{a, b}\right|$ for any $b \in[2 r]$; and for each $y \in N \cap\left(\psi^{\prime}\right)^{-1}(a, b),(\mathscr{T} 3)$ guarantees a corresponding set $C_{y} \subseteq X_{\psi^{*}(y)} \backslash g_{2}\left(X^{\prime}\right)=U_{\psi^{\prime}(y)}=U_{a, b}$ that has size at least $\mathrm{cm} / 2 \geq c\left|U_{a, b}\right| / 3$. Let $H_{a}$ denote the subgraph of $H$ induced by the set of all $x \in V(H) \backslash\left(X^{\prime} \cup I\right)$ 
such that $\psi^{\prime}(x)=(a, b)$ for some $b \in[2 r]$. Now apply, for each $a \in[2 \ell]$, Lemma 4.6 (blow-up lemma) with $G^{\prime}\left[\bigcup_{b \in[2 r]} U_{a, b}\right]$ and $H_{a}$ playing the roles of $G$ and $H$ and $2 \varepsilon^{1 / 27}, 2 \varepsilon, \delta / 5, c / 3, \Delta, 2 r,\left\{U_{a, b}: b \in\right.$ $[2 r]\}, \psi^{\prime}, C_{y}$ playing the roles of $\varepsilon, \alpha, \delta, c, \Delta, k,\left\{V_{a}: a \in[k]\right\}, \phi, S_{y}$. Altogether, this yields a mapping

$$
g_{3}: V(H) \backslash\left(X^{\prime} \cup I\right) \rightarrow V\left(G^{\prime}\right) \backslash\left(V_{0} \cup g_{2}\left(X^{\prime}\right)\right)
$$

that is an embedding of $H \backslash\left(X^{\prime} \cup I\right)$ into $V\left(G^{\prime}\right)$ such that every $y \in N$ is mapped to a vertex in $C_{y}$.

We claim that the mapping $g$ given by

$$
g(x)= \begin{cases}g_{1}(x) & \text { if } x \in I \\ g_{2}(x) & \text { if } x \in X^{\prime} \\ g_{3}(x) & \text { otherwise }\end{cases}
$$

is an embedding of $H$ into $G^{\prime}$ (and hence into $G$ ).

Firstly, $g$ is an injective map from $V(H)$ to $V\left(G^{\prime}\right)$ by the definitions of $g_{1}, g_{2}, g_{3}$. So we just need to check that it is a graph homomorphism. Also, by their definitions, each of $g_{1}, g_{2}, g_{3}$ is an embedding of $H$ induced on its respective domain into $G^{\prime}$. So it suffices to check that whenever $x y \in E(H)$ and $x, y$ are not both in $I$ or in $X^{\prime}$ or in $V(H) \backslash\left(X^{\prime} \cup I\right), g(x) g(y) \in E\left(G^{\prime}\right)$.

Suppose first that $x \in I$ and $y \in V(H) \backslash I$. Then $g(x)=g_{1}(x)=\psi(x)$ and $y \in W \subseteq X^{\prime}$ (here we used Claim 8.2(v)). So $g(y)=g_{2}(y)$. Claim 8.2(iii) implies that $x$ is the only vertex in $I$ that is a neighbour of $y$. Then

$$
g(y) \stackrel{(8.26)}{=} g_{2}(y) \stackrel{(\mathscr{T} 2)}{\in} S_{y} \stackrel{(8.24)}{=} N_{G^{\prime}}\left(\psi(x), X_{\psi(y)}\right)=N_{G^{\prime}}\left(g(x), X_{\psi(y)}\right) .
$$

So $g(x) g(y) \in E\left(G^{\prime}\right)$, as required.

Therefore, we may assume that $x \in X^{\prime}$ and $y \in V(H) \backslash\left(X^{\prime} \cup I\right)$. Then $g(x)=g_{2}(x), y \in N$, and $g(y)=g_{3}(y) \in C_{y}$, where $C_{y}$ was defined in $(\mathscr{T} 3)$, which guarantees that $C_{y} \subseteq N_{G^{\prime}}\left(g_{2}(x)\right)=$ $N_{G^{\prime}}(g(x))$. So $g(x) g(y) \in E\left(G^{\prime}\right)$, as required. This completes the proof of Theorem 1.2.

\section{Concluding remarks}

In this paper, we prove a version of the bandwidth theorem for locally dense graphs. As mentioned in the introduction, it is also of interest to seek minimum degree conditions that force a given spanning structure in a graph with sublinear independence number. In particular, it would be very interesting to obtain an analogue of the bandwidth theorem in this setting.

In a step in this direction, Balogh, Molla, and Sharifzadeh [4] proved the following result on triangle factors.

Theorem 9.1 (Balogh, Molla, and Sharifzadeh [4]). For every $\varepsilon>0$, there exist $\gamma>0$ and $n_{0} \in \mathbb{N}$ such that the following holds. For every $n$-vertex graph $G$ with $n \geq n_{0}$ divisible by 3 , if $\delta(G) \geq(1 / 2+\varepsilon) n$ and $G$ has independence number $\alpha(G) \leq \gamma n$, then $G$ has a $K_{3}$-factor.

Perhaps the next natural step is to ascertain whether the conclusion of Theorem 9.1 can be strengthened to ensure the square of a Hamilton cycle.

Conjecture 9.2. For every $\varepsilon>0$, there exist $\gamma>0$ and $n_{0} \in \mathbb{N}$ such that the following holds. For every $n$-vertex graph $G$ with $n \geq n_{0}$, if $\delta(G) \geq(1 / 2+\varepsilon) n$ and $\alpha(G) \leq \gamma n$, then $G$ contains the square of a Hamilton cycle.

It is also natural to seek a version of Theorem 1.2 where now one replaces the condition of locally dense with a more restrictive uniformly dense condition: given $\rho, d>0$, we say that an $n$-vertex graph $G$ is $(\rho, d)$-uniformly dense if every $X, Y \subseteq V(G)$ satisfies $e_{G}(X, Y) \geq d|X||Y|-\rho n^{2}$. If one restricts to uniformly dense graphs, then one can substantially reduce the minimum degree condition in Theorem 1.2, as well as remove the bandwidth condition on $H$. 
Theorem 9.3. For all $\Delta \in \mathbb{N}$ and $d, \eta>0$, there exist constants $\rho, n_{0}>0$ such that for every $n \geq n_{0}$, the following holds. Let $H$ be an $n$-vertex graph with $\Delta(H) \leq \Delta$. Then any $(\rho, d)$-uniformly dense graph $G$ on $n$ vertices with $\delta(G) \geq \eta n$ contains a copy of $H$.

Theorem 9.3 can be proven by a simple application of the blow-up lemma; a more general 'rainbow' version of Theorem 9.3 is given in [20, Corollary 1.3].

Acknowledgements. Katherine Staden was supported by ERC grant 306493, and Andrew Treglown was supported by EPSRC grant EP/M016641/1.

We would like to thank Maryam Sharifzadeh for many helpful conversations at the start of the project. Andrew Treglown is grateful to Stefan Glock and Felix Joos for a conversation on [20] that brought to light the version of the bandwidth theorem for uniformly dense graphs.

The authors are also grateful to the referees for their helpful and careful reviews.

Conflict of Interest: None.

\section{References}

[1] P. Allen, J. Böttcher, J. Ehrenmüller, and A. Taraz, 'The bandwidth theorem in sparse graphs', Advances Combin. (2020):6, 60pp.

[2] J. Balogh and J. Lenz, 'Some exact Ramsey-Turán numbers', Bull. London Math. Soc. 44 (2012), 1251-1258.

[3] J. Balogh, A. McDowell, T. Molla, and R. Mycroft, 'Triangle-tilings in graphs without large independent sets', Combin. Probab. Comput. 27 (2018), 449-474.

[4] J. Balogh, T. Molla, and M. Sharifzadeh, 'Triangle factors of graphs without large independent sets and of weighted graphs', Random Structures Algorithms 49 (2016), 669-693.

[5] J. Böttcher, Y. Kohayakawa, and A. Taraz, 'Almost spanning subgraphs of random graphs after adversarial edge removal', Combin. Probab. Comput. 22 (2013), 639-683.

[6] J. Böttcher, R. Montgomery, O. Parczyk, and Y. Person, 'Embedding spanning bounded degree graphs in randomly perturbed graphs', Mathematika 66 (2020), 422-447.

[7] J. Böttcher, K. Preussmann, A. Taraz, and A. Würfl, 'Bandwidth, expansion, treewidth, separators and universality for bounded-degree graphs', European J. Combin. 31 (2010), 1217-1227.

[8] J. Böttcher, M. Schacht, and A. Taraz, 'Spanning 3-colourable subgraphs of small bandwidth in dense graphs', J. Combin. Theory B 98 (2008), 752-777.

[9] J. Böttcher, M. Schacht, and A. Taraz, 'Proof of the bandwidth conjecture of Bollobás and Komlós', Math. Ann. 343 (2009), 175-205.

[10] J. Böttcher, A. Taraz, and A. Würfl, 'Spanning embeddings of arrangeable graphs with sublinear bandwidth', Random Structures Algorithms, 48 (2016), 270-289.

[11] V. Chvátal, V. Rödl, E. Szemerédi, and W.T. Trotter Jr., 'The Ramsey number of a graph with bounded maximum degree', J. Combin. Theory Ser. B 34 (1983), 239-243.

[12] P. Condon, J. Kim, D. Kühn, and D. Osthus, 'A bandwidth theorem for approximate decompositions', Proc. London Math. Soc. 118 (2019), 1393-1449.

[13] G.A. Dirac, 'Some theorems on abstract graphs', Proc. London Math. Soc. 2 (1952), 69-81.

[14] O. Ebsen, G.S. Maesaka, Chr. Reiher, M. Schacht, and B. Schülke, 'Embedding spanning subgraphs in uniformly dense and inseparable graphs', Random Structures and Algorithms, 57 (2020), 1077-1096.

[15] P. Erdős, Problem 9, in: M. Fieldler (ed.), Theory of Graphs and Its Applications (Czech. Acad. Sci. Publ., Prague, 1964), 159.

[16] P. Erdős, R.J. Faudree, C.C. Rousseau, and R.H. Schelp, 'A local density condition for triangles', Discrete Math. 127 (1994), 153-161.

[17] P. Erdôs, A. Hajnal, V.T. Sós, and E. Szemerédi, 'More results on Ramsey-Turán type problems', Combinatorica 3 (1983), 69-81.

[18] P. Erdős and V.T. Sós, 'Some remarks on Ramsey's and Turán’s theorem', in: Combinatorial Theory and Its Applications vol. II (North-Holland, Amsterdam, 1970), 395-404.

[19] A. Ferber, R. Nenadov, A. Noever, U. Peter, and N. Skoric, 'Robust hamiltonicity of random directed graphs', J. Combin. Theory Ser. B 126 (2017), 1-23.

[20] S. Glock and F. Joos, 'A rainbow blow-up lemma', Random Structures Algorithms, to appear.

[21] A. Hajnal and E. Szemerédi, 'Proof of a conjecture of Erdős', in: Combinatorial Theory and Its Applications vol. II 4 (North-Holland, Amsterdam, 1970), 601-623.

[22] J. Han, 'On perfect matchings and tilings in uniform hypergraphs', SIAM J. Discrete Math., 32 (2018), 919-932.

[23] H. Huang, C. Lee, and B. Sudakov, 'Bandwidth theorem for random graphs', J. Combin. Theory B 102 (2012), 14-37. 
[24] F. Knox and A. Treglown, 'Embedding spanning bipartite graphs of small bandwidth', Combin. Probab. Comput. 22 (2013), 71-96.

[25] Y. Kohayakawa, B. Nagle, V. Rödl, and M. Schacht, 'Weak regularity and linear hypergraphs', J. Combin. Theory B 100 (2010), 151-160.

[26] J. Komlós, 'The blow-up lemma', Combin. Probab. Comput. 8 (1999), 161-176.

[27] J. Komlós, G.N. Sárkozÿ, and E. Szemerédi, 'Blow-up lemma', Combinatorica 17 (1997), 109-123.

[28] J. Komlós, G.N. Sárközy, and E. Szemerédi, 'Proof of the Seymour conjecture for large graphs', Annals of Combinatorics 2 (1998), 43-60.

[29] D. Kühn and D. Osthus, 'The minimum degree threshold for perfect graph packings', Combinatorica 29 (2009), $65-107$.

[30] C. Lee, 'Embedding degenerate graphs of small bandwidth', submitted, arXiv:1501.05350.

[31] D. Mubayi and V.T. Sós, 'Explicit constructions of triple systems for Ramsey-Turán problems', J. Graph Theory 52 (2006), 211-216.

[32] V. Rödl, A. Ruciński, and E. Szemerédi, 'A Dirac-type theorem for 3-uniform hypergraphs', Combin. Probab. Comput. 15 (2006), 229-251.

[33] P. Seymour, Problem section, in: T.P. McDonough and V.C. Mavron (eds.), Combinatorics: Proceedings of the British Combinatorial Conference 1973 (Cambridge University Press, 1974), 201-202.

[34] M. Simonovits and V. T. Sós, 'Ramsey-Turán theory', Discrete Math. 229 (2001), 293-340.

[35] K. Staden and A. Treglown, 'On degree sequences forcing the square of a Hamilton cycle', SIAM J. Disc. Math. 31 (2017), 383-437.

[36] K. Staden and A. Treglown, 'On degree sequences forcing the square of a Hamilton cycle', arXiv version, arXiv:1412.3498.

[37] E. Szemerédi, 'Regular partitions of graphs', Problémes Combinatoires et Théorie des Graphes Colloques Internationaux CNRS 260 (1978), 399-401. 